\title{
Yet Another Hydrodynamic Model with Correlated Parameters for Silicon Devices
}

\author{
Muhammad H. El-Saba \\ Ain-Shams University, Faculty of Engineering, Dept of Electronic En gineering, Cairo, Egy pt
}

\begin{abstract}
In this paper we present a complete hydrodynamic model (HDM) describing the transport of charge carriers in semiconductor devices with arbitrary band structure. The model is appended with advanced physical models for almost all the physical para meters of interest in modern Si Devices. These para meters are inter-related via the carrier energy relaxation time. An improved analytical model of the carrier heat flux, on the basis of a fourth moment of the Boltzmann transport equation (BTE), is also presented. In order to resolve the heat evacuation problems in SOI and power devices, the model is coupled with a lattice heat conservation equation. The transport of hot carriers is simu lated, according to the proposed HDM and the results are compared with the conventional data obtained using the drift-diffusion model (DDM) and MC simulation. We show from the HD simulation, the effect of the energy relaxation time value on the hot carrier transport in general and the breakdown voltage in particular, of both MOSFETs and bipolar devices.
\end{abstract}

Keywords Semiconductor Devices, Hydrodynamic Modelling, Simulation, Semiclssical Transport Theory, Impact Ionization, Injection, Hot Carriers

\section{Introduction}

For a gas of charge carriers of q-type, where $q$ stands for $(n)$ for electrons or $(p)$ for holes, the semiclassical Boltzmann transport equation (BTE) may be written as follows, when charge carriers are not strongly correlated [1]

$$
\partial \mathrm{f}_{\mathrm{q}} / \partial \mathrm{t}+\mathrm{u}_{\mathrm{q}} . \nabla \mathrm{f}_{\mathrm{q}}-(\mathbf{F} / \mathrm{h}) \cdot \nabla_{\mathrm{k}} \mathrm{f}_{\mathrm{q}}=\left(\partial \mathrm{f}_{\mathrm{q}} / \partial \mathrm{t}\right)_{\mathrm{col}}
$$

where $f_{q}=f_{q}(\boldsymbol{k}, \boldsymbol{x}, t)$ is the carrier distribution function of the q-type gas in the phase space, $u_{q}$ is the group velocity of carriers, $\boldsymbol{F}= \pm e\left(\boldsymbol{\zeta}+u_{q} x \boldsymbol{B}\right)$ is the applied force due to electric field $\zeta$ and magnetic fie ld $\boldsymbol{B}$ and $\left(\partial_{q} / \partial\right)_{c o l}$ is the rate of change of the distribution function due to the internal collisions between the charge carriers and the crystal lattice (phonons and other defects).

Since the validity of the semiclassical transport approaches for simulating extremely scaled devices (sub 0.1 $\mu \mathrm{m}$ ) and $\mathrm{u}$ ltra fast devices (working up to the terahert $\mathrm{z}$ range) has been lately a subject of debate, we just remind here the main assumptions on which the BTE in semiconductors is based[2].

- Collisions are localized in space, so that the mean free path of charge carriers is much greater than the de-Brouglie wavelength,

- Collisions are instantaneous in time such that the collision time is much smaller than the time between

* Corresponding author:

mhs1308@hotmail.com (Muhammad H. El-Saba)

Published online at http://journal.sapub.org/msse

Copyright (C) 2012 Scientific \& Academic Publishing. All Rights Reserved subsequent collisions,

- External electric field force does not vary greatly over small distances in the order of wave packet length,

Particles are weakly correlated (Bogoliubov assumption [3]) and the many body effect is negligible.

Mathematically speaking, the BTE is valid as long as the following Barker condition is realized[4]:

$$
\left|\frac{e \varsigma_{m} \hbar q \tau_{c}}{2 m^{*}}\right| \leq \frac{2 \hbar}{\tau_{c c}}
$$

where $\zeta_{m}$ is the maximum applied electric field, $m^{*}$ is the carrier effective mass, $\tau_{c c}$ is collision time and $\tau_{c}$ is mean free time between collisions and $q=k-k$ ' is the wave vector of (emitted or absorbed) phonons during collisions.

For strongly ionized gasses and degenerate semiconductors, where particle-particle collisions are not negligible, the Boltzmann transport equation may be replaced by the Fokker-Planck equation[5-6]. For ultra small devices, whose active layers dimensions (at least one of them) are in the order of De Broglie wavelength, the BTE should be replaced by one of the quantum transport formulations [7-11], like the Wigner-Boltzmann transport equation (WBTE) [9-11]. However, there exist several approaches to include the degeneracy effects and quantum corrections to the semic lassical trans port models (e.g., [12-13]).

The solution of semiclassical BTE is carried out by a variety of methods[14-26], among which the iterative methods[15], the matrix methods[16], the Monte Carlo method[17-21], the cellu lar automata method[22], truncated expansion (usually in Legendre polynomials and spherical 
harmonics) methods[23-26]. In addition, the so-called energy transport model (ETM) and moment methods [27], which are sometimes called the hydrodynamic model (HDM), have been also used to solve the BTE in the hot carrier regime. In the ETM approach the distribution function is decomposed into an even and odd parts and the collision term is expressed using a microscopic relaxation time approximation[28].

The hydrodynamic description of the gas of charge carrier in semiconductors consists in the study of the evolution of certain macroscopic quantities, whose physical meaning is significant and whose quantity is eventually measurable. So, the HDM is constructed by multiplying the semiclassical BTE with a number of weight functions and integrating these equations over the phase or mo mentum space, so that a set of differential equations in physical space and time is obtained. This method is hence an averaging process that leads to the loss of some microscopic information in the distribution function. However, in many practical cases the resultant average (or macroscopic) parameters retained by the equations in the physical space are sufficient to capture the essential features of the carrier transport phenomena. The HDM has been widely used in the study of aerodynamic flow, and was suggested to study the hot electron transport in multivalley semiconductor devices by Bløtekjaer [29]. Since then, several contributions have been introduced to improve the hot carrier transport models in semiconductors and the HDM in particular[30-47]. For instance, the nonparabolicity of energy bands has been included in the HDM for the first time by Thoma et al[38]. The energy transport model ETM was reformulated by Chen, Kan and Ravaioli, who assumed non-Maxwellian and nonparabolicity correction factors, to solve the contradiction involved in evaluating a non-zero heat flux term for a Maxwellian distribution[39]. Also, the number of considered moments in the HDM has been increased in order to improve the HDM accuracy[44]. Unfortunately, all the added improvements to the HDM were associated with the addition of a huge number of new physical para meters [45-46]. On the other hand, so me authors neglected the role of some important parameters in order to reduce the complexity of the model. This led to several paradoxal results and the failure of the HDM, in certain situations[47]. We try here to generalize the previously introduced models while reducing the number of physical parameters, by correlating them all to the energy relaxation time. We start by introducing the system of hydrodynamic equations in section II. In section III we discuss the system closure hypotheses. We present the physical definition of the carrier temperature. In section IV we present an almost complete description of the physical parameters in the bulk of semiconductor devices. Finally we present our conclusions in section VIII.

\section{System of Hydrodynamic Equations}

The multiplying weight functions in the HDM for semiconductors are usually chosen as powers of increasing order of the carrier vector $\boldsymbol{k}_{q}$, or carrier group velocity $\boldsymbol{u}_{q}$, or even the carrier momentum $\boldsymbol{p}_{q}$. The powers of these parameters are usually formatted using some appropriate scaling factors to get physically meaningful quantities. For instance, if we adopt the powers of the carrier group velocity as weight functions (e.g., $1, \boldsymbol{u}_{q}$ and $u_{q}{ }^{2}$ ), then we use the carrier energy $E_{q}=1 / 2 m_{q}{ }^{*} u_{q}{ }^{2}$, instead of $u_{q}{ }^{2}$ as the second-order multiplier. So, the set of conservation equations of carrier concentration, momentum and energy may be obtained by multiplying the BTE by the first powers of the carrier group velocity $\boldsymbol{u}_{q}\left(\mathrm{e} . \mathrm{g} ., 1, \boldsymbol{u}_{q}\right.$ and $E_{q}$ ), and then integrating the two sides of the resulting equations over the whole k-space (see Appendix I). For the sake of simplicity and brevity we write here only the electron equations in a single-valley semiconductor. In multi-valley semiconductors, we may assume a single lumped valley or write similar equations for each valley of the semiconductor[37]. The electron density-, momentum- and energy- conservation equations, in a semiconductor, can be put in the following form:

$$
\begin{gathered}
\partial \mathrm{n} / \partial \mathrm{t}+\nabla \cdot\left(\mathrm{n} \mathbf{v}_{\mathrm{n}}\right)=[\partial \mathrm{n} / \partial \mathrm{t}]_{\mathrm{col}} . \\
\partial\left(\mathrm{n} \mathbf{v}_{\mathrm{n}}\right) \partial \mathrm{t}+\nabla \cdot\left(\mathrm{m}_{\mathrm{n}}{ }^{-1} \mathbb{P}_{\mathrm{n}}\right)+\nabla \cdot\left(\mathrm{n} \mathbf{v}_{\mathrm{n}} \otimes \mathbf{v}_{\mathrm{n}}\right) \\
=\mathrm{n} \mathrm{m}_{\mathrm{n}}^{-1} \cdot \mathbf{F}+\left[\partial\left(\mathrm{n} \mathbf{v}_{\mathrm{n}}\right) \partial \mathrm{t}\right]_{\mathrm{col}} \\
\partial\left(\mathrm{n} \omega_{\mathrm{n}}\right) / \partial \mathrm{t}+\nabla \cdot \mathbf{S}_{\mathrm{n}}=\mathrm{n} \mathbf{v}_{\mathrm{n}} \cdot \mathbf{F}+\left[\partial\left(\mathrm{n} \omega_{\mathrm{n}}\right) / \partial \mathrm{t}\right]_{\mathrm{col}} .
\end{gathered}
$$

where $n, \boldsymbol{v}_{n}, \boldsymbol{P}_{n}$ and $\omega_{n}$ are the electron concentration, mean velocity, electrokinetic pressure tensor and mean energy, respectively. Also, $\boldsymbol{S}_{n}$ is the electron energy flow and $m_{n}{ }^{-1}$ is the mean inverse mass of electrons. We dully note that we don't adopt the effective mass approximation here, and the second derivative of electron energy just appears as a result of the averaging process in the k-space (Appendix I). Therefore, the mean inverse mass of carriers is generally a tensor whose components $m_{n}{ }^{-1}(i, j)=\left(1 / \hbar^{2}\right)\left\langle\partial^{2} E_{n} / \partial k_{i} \partial k_{j}\right\rangle$ are supposed functions of the mean carrier energy. When the energy band structure of the semiconductor material is assumed parabolic, $m_{n}^{-1}$ is a constant and equal to the conventional electron inverse effective masse $\left(m_{n} *\right)^{-1}$ at the bottom of conduction band. However, in this work we consider $m_{n}$ as a scalar quantity averaged over the semiconductor valleys $m_{n}{ }^{-1}=1 / 3 \sum m_{n}{ }^{-1}(i, j)$. At the bottom of conduction band the average mass will be denoted by $m_{n o}$ and its value in $\mathrm{Si}$, according to optical measurements is about $0.275 m_{o}$. The subscript 'col.' In the above equations means the rate of change of the quantity between brackets due to electron gas collisions with the semiconductor crystal lattice and its defects. The sign $\otimes$ stands for the tensorial product of two terms such that:

$$
\nabla .\left(\mathrm{n} \mathbf{v}_{\mathrm{n}} \otimes \mathbf{v}_{\mathrm{n}}\right)=\mathbf{v}_{\mathrm{n}} \nabla \cdot\left(\mathrm{n} \mathbf{v}_{\mathrm{n}}\right)+\left(\mathrm{n} \mathbf{v}_{\mathrm{n}} \cdot \nabla\right) \mathbf{v}_{\mathrm{n}} .
$$

The electrokinetic pressure tensor in the above equations is defined as follows:

$$
\mathbb{P}_{\mathrm{n}} \equiv \mathrm{n} \mathrm{m}_{\mathrm{n}}<\left(\mathbf{u}_{\mathrm{n}}-\mathbf{v}_{\mathrm{n}}\right) \otimes\left(\mathbf{u}_{\mathrm{n}}-\mathbf{v}_{\mathrm{n}}\right)>
$$

We discuss the significance of this definition in the following section. The carrier energy flow (or energy flux) is given by the following expression, for electrons:

$$
\mathbf{S}_{\mathrm{n}}=\mathrm{n}\left\langle\mathrm{E}_{\mathrm{n}} \mathrm{u}_{\mathrm{n}}\right\rangle=\mathbf{Q}_{\mathrm{n}}+\left(\mathrm{n} \omega_{\mathrm{n}}+\mathbb{P}_{\mathrm{n}}\right) \cdot \mathbf{v}_{\mathrm{n}}
$$


where $\boldsymbol{Q}_{n}$ is called the electron heat flux. In the effective mass approximation, where we express the electron energy by $E_{n}=1 / 2 m_{n}{ }_{n} u_{n}{ }^{2}$, the electron heat flux vector $\boldsymbol{Q}_{n}$ is defined as the third central moment of the electron distribution function $f_{n}$ :

$$
\begin{array}{ll}
\left.\mathbf{Q}_{\mathrm{n}}=\mathrm{n}<1 / 2 \mathrm{~m}_{\mathrm{n}}{ }^{*}\left(\mathbf{u}_{\mathrm{n}}-\mathbf{v}_{\mathrm{n}}\right) \cdot\left|\mathbf{u}_{\mathrm{n}}-\mathbf{v}_{\mathrm{n}}\right|^{2}\right)> \\
=1 / 2 \int \mathrm{m}_{\mathrm{n}}^{*}\left|\mathbf{u}_{\mathrm{n}}-\mathbf{v}_{\mathrm{n}}\right|^{2}\left(\mathbf{u}_{\mathrm{n}}-\mathbf{v}_{\mathrm{n}}\right) \cdot \mathrm{f}_{\mathrm{n}}(\mathbf{x}, \mathbf{k}, \mathrm{t}) \mathrm{d}^{3} \mathbf{k}
\end{array}
$$

So, the heat flux component $\boldsymbol{Q}_{n(i)}$ is the flux of random thermal energy across a surface with its normal oriented in the $i^{\text {th }}$ direction. However, as the electron distribution function is not known we don't make use of this equation in our model. We dully note that the formal definition of the gas pressure tensor $\boldsymbol{P}_{n,(\mathrm{ij})}$ is the flux of the $i^{\text {th }}$ component of the momentum across a surface element moving with the mean velocity oriented with its normal in the $j^{\text {th }}$ direction[48], such that:

$$
\mathbb{P}_{\mathrm{n}(\mathrm{i}, \mathrm{j})} \equiv<\rho_{\mathrm{n}(\mathrm{ij})}\left(\mathbf{u}_{\mathrm{ni}}-\mathbf{v}_{\mathrm{ni}}\right)\left(\mathbf{u}_{\mathrm{nj}}-\mathbf{v}_{\mathrm{nj}}\right)>
$$

where $\rho_{n(i, j)}$ is the mass density of the gas. In the ideal gaz theory, the gaz mass density is a macroscopic quantity and $\rho_{n}$ is taken outside the averaging brackets. So, the gas mass density is given by:

$$
\begin{gathered}
\rho_{\mathrm{n}(\mathrm{i}, \mathrm{j})}=\mathrm{nm}_{\mathrm{n}(\mathrm{ij})}, \text { with } \mathrm{m}_{\mathrm{n}(\mathrm{ij})}{ }^{-1} \\
=\left(1 / \hbar^{2}\right) \int\left[\partial^{2} \mathrm{E}_{\mathrm{n}} / \partial \mathrm{k}_{\mathrm{i}} \partial \mathrm{k}_{\mathrm{j}}\right] \mathrm{f}_{\mathrm{n}}(\mathbf{x}, \mathbf{k}, \mathrm{t}) \mathrm{d}^{3} \mathbf{k} / \int \mathrm{f}_{\mathrm{n}}(\mathbf{x}, \mathbf{k}, \mathrm{t}) \mathrm{d}^{3} \mathbf{k}
\end{gathered}
$$

such that $\boldsymbol{P}_{n} \equiv \boldsymbol{P}_{n}$. However, in clasical transport models, the electron gas mass density is usually re lated to the electron effective mass such that $\rho_{n}=n m_{n}{ }^{*}$. Hence, our definition of the gas pressure, $\boldsymbol{Z}_{n}$, is different from the classic definition of electron gas pressure tensor, $\boldsymbol{P}_{n}$ :

$$
\mathbb{P}_{\mathrm{n}}=\mathrm{n}\left\langle\mathrm{m}_{\mathrm{n}}{ }^{*}\left(\mathbf{u}_{\mathrm{n}}-\mathbf{v}_{\mathrm{n}}\right) \otimes\left(\mathbf{u}_{\mathrm{n}}-\mathbf{v}_{\mathrm{n}}\right)\right\rangle
$$

Therefore, the electrokinetic pressure tensor $\boldsymbol{P}_{n}$ may be related to the classic definition $\boldsymbol{P}_{n}$ via a correction factor, $g_{n}$, such that:

$$
\begin{aligned}
\mathbf{g}_{\mathrm{n}}= & \left.\mathbb{P}_{\mathrm{n}} \cdot \mathbb{P}_{\mathrm{n}}^{-1}=\mathrm{C}_{<}<\mathrm{m}_{\mathrm{n}}{ }^{*}\left(\mathbf{u}_{\mathrm{n}}-\mathbf{v}_{\mathrm{n}}\right) \otimes\left(\mathbf{u}_{\mathrm{n}}-\mathbf{v}_{\mathrm{n}}\right)>\right] . \\
& {\left[\mathrm{m}_{\mathrm{n}}<\left(\mathbf{u}_{\mathrm{n}}-\mathbf{v}_{\mathrm{n}}\right) \otimes\left(\mathbf{u}_{\mathrm{n}}-\mathbf{v}_{\mathrm{n}}\right)>\right]^{-1} }
\end{aligned}
$$

We call this factor, the electron gas pressure $g$-factor. This factor can be calculated from MC simulation in the bulk of semiconductor with arb itrary band structure.

In absence of magnetic field, the applied force on electrons is given by $\boldsymbol{F}=-e \boldsymbol{\zeta}$, where the electric field $\boldsymbol{\zeta}$ is equal to the negative gradient of the electrostatic potential $\psi$. In heterogeneous devices, the applied force on electrons is given by $\boldsymbol{F}=-\nabla E_{c}$, where $E_{c}=\left(E_{c o}-e \psi\right)$ and $E_{c o}$ is the edge of the conduction band. Similarly, the applied force on holes is $\boldsymbol{F}=\nabla E_{v}$, where $E_{v}=\left(E_{v o}+e \psi\right)$ and $E_{v o}$ being the edge of the valence band. The electrostatic potential distribution can be found by solving the Poisson equation:

$$
\nabla .(-\varepsilon \nabla \psi+\mathrm{P})=\mathrm{e}\left(\mathrm{p}-\mathrm{n}+\text { Dop }+\mathrm{N}_{\mathrm{t}}\right)
$$

where $p$ is the hole concentration, $\varepsilon$ is the dielectric constant, $P$ is the polarization vector, $N_{t}$ is the density of traps and Dop $=\left|N_{d}{ }^{+}-N_{a}\right|$ is the net ionized concentration in the semiconductor.

\section{Closing the System of Hydrodynamic Equations}

In order to close the system of conservation equations for charge carrier density, average momentum and average energy $(2-a, b, c)$ we usually adopt some assumptions to approximate the terms which depend on the knowledge of the distribution function. The main assumptions are usually concerned with the following items:

1. definition of the carrier gas pressure and temperature,

2. approximation of collision terms,

3. modeling the heat flux term (the third center moment of the distribution function), and

4. modeling any other higher mo ment terms, if the number of moments is greater than 3 .

When the kinetic pressure of the gas of electrons is considered as a scalar quantity, one can define the electron gas temperature, $\boldsymbol{T}_{n}$, by the law of perfect gases $\boldsymbol{P}_{n}=n k_{B} \boldsymbol{T}_{n}$. This assumption does not imply a spherically-symmetric distribution function about its mean value in the momentum space (like the displaced Maxwellian distribution). Equation (5-a) can be merged with the hypothesis of the perfect gas law, in one relation defining the electron gas temperature as follows:

$$
\mathrm{k}_{\mathrm{B}} \mathbb{T}_{\mathrm{n}}=\left\langle\mathrm{m}_{\mathrm{n}}{ }^{*}\left(\mathbf{u}_{\mathrm{n}}-\mathbf{v}_{\mathrm{n}}\right) \otimes\left(\mathbf{u}_{\mathrm{n}}-\mathbf{v}_{\mathrm{n}}\right)\right\rangle
$$

This coincides with the physical definition of the temperature as a measure of the average kinetic energy of the gas particles [49]. Using the above definitions of the electron gas pressure and temperature, we can easily prove that the mean electron energy $\omega_{n}$ is related to the scalar electron temperature $T_{n}$ and scalar average mass $m_{n}$ as follows:

$\omega_{\mathrm{n}}=\left\langle\mathrm{E}_{\mathrm{n}}\right\rangle=\left\langle 1 / 2 \mathrm{~m}_{\mathrm{n}} * \mathrm{u}_{\mathrm{n}}^{2}\right\rangle=1 / 2 \mathrm{~g}_{\mathrm{n}} \mathrm{m}_{\mathrm{n}} \mathrm{v}_{\mathrm{n}}^{2}+3 / 2 \mathrm{k}_{\mathrm{B}} \mathrm{T}_{\mathrm{n}}$ (8)

Although we make use of the expression $E_{n}=1 / 2 m_{n} * u_{n}^{2}$ in order to derive the above relation between $\omega_{n}$ and $T_{n}$, the eventual error due the effective mass approximation is absorbed in the gas pressure correction factor, which can be calculated by MC simulation. However, it is well known that the convection part of the the average electron energy is generally small co mpared the thermal part, except for device regions where the velocity overshoot is pronounced.

As for the collision terms, we make use of the macroscopic relaxation time approximation[31]. In this approximation, the collision term in the momentum conservation equation is expressed in terms of a macroscopic momentum relaxation time $\tau_{m n}$, as follows:

$$
\left[\partial \mathrm{n} \mathbf{v}_{\mathrm{n}} / \partial \mathrm{t}\right]_{\mathrm{col} .}=-\mathrm{n} \mathbf{v}_{\mathrm{n}} / \tau_{\mathrm{mn}}+\mathbf{v}_{\mathrm{n}}[\partial \mathrm{n} / \partial \mathrm{t}]_{\mathrm{col}}
$$

Also, the collision term in the energy conservation equation can be expressed in terms of a macroscopic energy relaxation time $\tau_{w n}$, as follows:

$$
\left.\left[\partial \mathrm{n} \omega_{\mathrm{n}} / \partial \mathrm{t}\right]_{\mathrm{col} .}=-\mathrm{n}\left(\omega_{\mathrm{n}}-\omega_{\mathrm{o}}\right) / \tau_{\mathrm{wn}}+\omega_{\mathrm{n}[} \partial \mathrm{n} / \partial \mathrm{t}\right]_{\mathrm{col}} .
$$

where $\omega_{o}=3 / 2 \quad k_{B} T_{L}$ is the electron energy at thermal equilibrium and $T_{L}$ is the lattice temperature. Note that the collision term in the above two equations is decomposed into two terms. So, the momentum and energy relaxation times, include the effect of collisions over optical while the effect of impact ionization collisions is included in the $[\partial / \partial]_{c o l}$. The collision term $\left[\partial{ }^{2} / \partial\right]_{c o l}$, which appears explicitly in the carrier density conservation equation can be expressed as follows: 


$$
[\partial \mathrm{n} / \partial \mathrm{t}]_{\mathrm{col}}=-\left(\mathrm{n}-\mathrm{n}_{\mathrm{o}}\right) / \tau_{\mathrm{n}}=-\mathrm{U}
$$

where $(U=R-G)$ is the net reco mbination-generation rate in the semiconductor. Note that the recombination rate, $R$, regroups the relevant recombination mechanisms in the semiconductor device (e.g., radiative, Shockley-Reed-Hall and Auger recombination mechanisms[50]). Also, the generation rate, $G$, regroups the applicable generation mechanis $\mathrm{ms}$ in the semiconductor device (e.g., thermal and optical generation as well as the generation by impact ionization mechanism).

Using the relaxation-time approximation and our definition of the carrier te mperature, the set of hydrodynamic equations (HDE's) can be formulated as follows for a single valley semiconductor:

$$
\text { e } \partial \mathrm{n} / \partial \mathrm{t}-\nabla \cdot \mathbf{J}_{\mathrm{n}}=\mathrm{e}(\mathrm{G}-\mathrm{R})
$$

$\mathbf{J}_{\mathrm{n}}-\mathrm{n} \mu_{\mathrm{n}} \mathrm{m}_{\mathrm{n}} \mathrm{d}\left[\mathbf{v}_{\mathrm{n}}\right] / \mathrm{dt}=\mathrm{e} \mathrm{n} \mu_{\mathrm{n}} \cdot \zeta+\mathrm{k}_{\mathrm{B}} \mu_{\mathrm{n}} \mathrm{m}_{\mathrm{n}} \nabla .\left(\mathrm{n} \mathbf{g}_{\mathrm{n}} \mathrm{m}_{\mathrm{n}}^{-1} \cdot \mathbb{T}_{\mathrm{n}}\right)(10 . \mathrm{b})$

$\partial\left(\mathrm{n} \omega_{\mathrm{n}}\right) / \partial \mathrm{t}+\nabla \cdot \mathbf{S}_{\mathrm{n}}=\zeta \cdot \mathbf{J}_{\mathrm{n}}+\omega_{\mathrm{n}}(\mathrm{G}-\mathrm{R})-\mathrm{n}\left(\omega_{\mathrm{n}}-\omega_{\mathrm{o}}\right) / \tau_{\mathrm{wn}} \quad(10 . \mathrm{c})$

The electron drift mobility $\mu_{n}$ is defined as follows:

$$
\mu_{\mathrm{n}}=\mathrm{e} \tau_{\mathrm{mn}} \mathrm{m}_{\mathrm{n}}^{-1}
$$

Similar equations can be written for holes. We dully note that the average electron energy $\omega_{n}$ is measured above the conduction band edge $E c$, while the average hole energy $\omega_{p}$ is measured under the valence band edge $E v$.

In steady state (up to the terahertz frequency range), the carrier momentum conservation equations can be further simp lified by suppressing the partial time-derivatives so that:

$$
\begin{gathered}
\mathbf{J}_{\mathrm{n}}-\mathrm{n} \mu_{\mathrm{n}} \cdot \mathrm{m}_{\mathrm{n}}\left(\mathbf{v}_{\mathrm{n}} \cdot \nabla\right)\left(\mathbf{v}_{\mathrm{n}}\right) \\
=\mathrm{e} \mathrm{n} \mu_{\mathrm{n}} \cdot \zeta+\mathrm{k}_{\mathrm{B}} \mu_{\mathrm{n}} \cdot \mathrm{m}_{\mathrm{n}} \nabla \cdot\left(\mathrm{n} \mathbf{g}_{\mathrm{n}} \mathrm{m}_{\mathrm{n}}^{-1} \cdot \mathbb{T}_{\mathrm{n}}\right) \\
\mathbf{J}_{\mathrm{p}}+\mathrm{p} \mu_{\mathrm{p}} \cdot \mathrm{m}_{\mathrm{p}}\left(\mathbf{v}_{\mathrm{p}} \cdot \nabla\right)\left(\mathbf{v}_{\mathrm{p}}\right) \\
=\mathrm{e} \mathrm{p} \mu_{\mathrm{p}} \cdot \zeta-\mathrm{k}_{\mathrm{B}} \mu_{\mathrm{p}} \cdot \mathrm{m}_{\mathrm{p}} \nabla \cdot\left(\mathrm{p} \mathbf{g}_{\mathrm{p}} \mathrm{m}_{\mathrm{p}}^{-1} \cdot \mathbb{T}_{\mathrm{p}}\right)
\end{gathered}
$$

The above conservation equations are similar to the current equations of the isothermal DDM, except for the acceleration and thermal diffusion terms. Also the carrier energy flow (or energy flux) can be put in the following forms, for electrons and holes:

$$
\begin{gathered}
\mathbf{S}_{\mathrm{n}}=\mathbf{Q}_{\mathrm{n}}-\left(\omega_{\mathrm{n}}+\mathbf{g}_{\mathrm{n}} \mathrm{k}_{\mathrm{B}} \mathbb{T}_{\mathrm{n}}\right) \cdot\left(\mathbf{J}_{\mathrm{n}} / \mathrm{e}\right) \\
\mathbf{S}_{\mathrm{p}}=\mathbf{Q}_{\mathrm{p}}+\left(\omega_{\mathrm{p}}+\mathbf{g}_{\mathrm{p}} \mathrm{k}_{\mathrm{B}} \mathbb{T}_{\mathrm{p}}\right) \cdot\left(\mathbf{J}_{\mathrm{p}} / \mathrm{e}\right)
\end{gathered}
$$

As indicated by (4-b), the carrier heat flu $x$ terms is defined in terms of the carrier distribution function, which is unknown. Therefore, the carrier-heat flux is usually modeled by the Fourier relation [51]:

$$
\mathbf{Q}_{\mathrm{n}}=-\mathrm{k}_{\mathrm{n}} \nabla \mathrm{T}_{\mathrm{n}}
$$

where $k_{n}$ is the electron thermal conductivity. The carrier thermal conductivity is usually modeled by the W iedemann Franz law, which states:

$$
\mathrm{k}_{\mathrm{n}}=\gamma_{\mathrm{n}}\left(\mathrm{k}_{\mathrm{B}} / \mathrm{e}\right)^{2} \mathrm{~T}_{\mathrm{n}} \sigma_{\mathrm{n}}
$$

where $\sigma_{n}$ is the electrical conductivity and $\gamma_{n}$ is the Lorenz number (for electrons). In the original Wiedeman-Franz formulation (using the microscopic relaxation time approximation) $\gamma_{n}$ is given by:

$$
\gamma_{n}=5 / 2+r
$$

where $r$ is an exponent in the mic roscopic relaxation time $\tau_{D n}$ expression, which is given by:

$$
\tau_{\text {Dn }}=\tau_{\mathrm{o}}\left(\mathrm{k}_{\mathrm{B}} \mathrm{T}_{\mathrm{L}} / \mathrm{E}_{\mathrm{n}}\right)^{\mathrm{r}}
$$

A more rigorous approach for modelling the carrier heat flux term consis ts in taking the third moment of the BTE into account. This procedure increases the number of differential equations to be solved and the number of parameters of the HDM. However, we show in section 5.4 how to derive a more accurate model of the carrier heat flux term and the Lorentz nu mber, on the bas is of the third moment of the BTE. We do this without increasing the number of differential equations of the HDM.

\section{About the Definition of the Carrier Temperature}

In this section we compare the different approaches for modeling the carrier temperature. Formally, temperature is a property which governs the transfer of thermal energy, or heat, between one system and another. The electronic temperature concept was initially introduced in 1947 by Fröhlich[52], and since then it has been exploited by several authors to model the high-field transport in semiconductors. In the isothermal drift-diffusion model (DDM), the carriers temperature is assumed equal to the lattice temperature $T_{L}$. However, the so-called static electron temperature $T_{n}$ may be roughly estimated from the electric field distribution in several ways [53]. For instance, the static te mperature may be defined as follows:

$\mathrm{T}_{\mathrm{n}}($ static $)=\mathrm{T}_{\mathrm{L}}\left(\mu_{\mathrm{no}} / \mu_{\mathrm{n}}(\zeta)\right)=\mathrm{T}_{\mathrm{L}}\left[1+\left(\mu_{\mathrm{no}} \zeta^{\prime \prime} / \mathrm{v}_{\mathrm{n}}{ }^{\mathrm{sat}}\right)^{2}\right]^{1 / 2}(13)$ where $\mu_{n}$ is the electron drift mobility, $v_{n}{ }^{\text {sat }}$ is the electron saturation velocity and $\mu_{n o}$ is the low-field drift mobility. Also, $\zeta^{\prime /}$ is the parallel component of the electric field to the electron current density such that $\zeta^{\prime \prime}=\zeta . J_{n} /\left|J_{n}\right|$. The above equation is based on the assumption that the parallel component of the diffusion coeffic ient is almost constant and independt of the electric field[33]. Such static forms of the carrier temperature exclude the lag effect between the electric field and the carrier temperature, and hence the velocity overshoot phenomenon will not be observed. However, the definition of the carrier temperature in the Monte Carlo simulation is given by:

$$
3 / 2 \mathrm{k}_{\mathrm{B}} \mathrm{T}_{\mathrm{n}}=\left\langle\mathrm{E}_{\mathrm{n}}(\mathrm{k})\right\rangle
$$

Also, the carrier temperature in the conventional hydrodynamic models, where the effective mass approximation is assumed and the anisotropy of the distribution function is ignored, is defined by:

$$
3 / 2 \mathrm{k}_{\mathrm{B}} \mathrm{T}_{\mathrm{n}}=1 / 2 \mathrm{~m}_{\mathrm{n}}{ }^{*}\left\langle\left|\mathbf{u}_{\mathrm{n}}-\mathbf{v}_{\mathrm{n}}\right|^{2}\right\rangle
$$

It is well known, that the above two definitions result in different temperatures[38]. In fact, the conventional hydrodynamic definition (14-b) is implicitly based on the displaced Maxwellian distribution at the electronic temperature.

$\mathrm{f}_{\mathrm{m}}(\mathrm{k})=\mathrm{A} \cdot\left[\mathrm{n} /\left(\mathrm{k}_{\mathrm{B}} \mathrm{T}_{\mathrm{n}}\right)^{3 / 2}\right] \cdot \exp \left(-1 / 2 \mathrm{~m}_{\mathrm{n}}{ }^{*}\left(\mathbf{u}_{\mathrm{n}}(\mathrm{k})-\mathbf{v}_{\mathrm{n}}\right)^{2} / \mathrm{k}_{\mathrm{B}} \mathrm{T}_{\mathrm{n}}\right)(15 \cdot \mathrm{a})$ where $A$ is a constant. Assuming that the collisions are fully rando mized, such that the drift energy may be neglected with respect to the thermal energy, then the electron distribution may be approximated to the so-called heated Maxwellian as follows: 
$f_{m}\left(E_{n}\right)=B \cdot\left[n /\left(k_{B} T_{n}\right)^{3 / 2}\right] \cdot \exp \left(-E_{n}(k) / k_{B} T_{n}\right) \quad(15 . b)$ where $E_{n}=1 / 2 m_{n}{ }^{*} \boldsymbol{u}_{n}^{2}$ is the electron energy and $B$ is constant. According to Staratton[28], the electronic temperature has no physical meaning, unless the distribution function is a displaced Maxwellian as described by equation (15). However, the Maxwellian distribution is only valid near equilibrium, and in highly doped regions, where carrier-carrier collisions are dominant. In fact, it has been confirmed by several authors (e.g.,[21]) that the energy distribution function is not Maxwellian at high electron energies. In the so-called improved energy transport model[39], the nonparabolicity of the band structure is taken into account and the distribution function $f_{n}(E)$ is appended by a non-Maxwellian factor $\gamma$, such that:

$$
f_{n}\left(E_{n}\right)=\left(1+\gamma \cdot E_{n} / k_{B} T_{n}\right) \cdot f_{m}\left(E_{n}\right)
$$

with $f_{m}(E)$ is the heated Maxwellian distribution function at an elevated temperature $T_{n}$. Then, a modified (or equivalent) carrier temperature $T_{m}$ is defined as follows:

$$
\mathrm{T}_{\mathrm{m}}=\mathrm{T}_{\mathrm{n}}(1+\gamma)
$$

However, referring to our system of HDE's (8), we make use of the formal definition for the electron temperature tens or:

$$
\mathrm{k}_{\mathrm{B}} \mathbb{T}_{\mathrm{n}}=\left\langle\mathrm{m}_{\mathrm{n}}^{*}\left(\mathbf{u}_{\mathrm{n}}-\mathbf{v}_{\mathrm{n}}\right) \otimes\left(\mathbf{u}_{\mathrm{n}}-\mathbf{v}_{\mathrm{n}}\right)\right\rangle
$$

When the carrier temperature tensor is reduced to a scalar quantity (such that $\boldsymbol{T}_{n}=T_{n} \boldsymbol{I}$ where $\boldsymbol{I}$ is the unity tensor), then the above definition may be simply written as follows:

$$
3 / 2 \mathrm{k}_{\mathrm{B}} \mathrm{T}_{\mathrm{n}}=1 / 2 \quad \mathrm{~g}_{\mathrm{n}} \mathrm{m}_{\mathrm{n}}\left\langle\left|\mathbf{u}_{\mathrm{n}}-\mathbf{v}_{\mathrm{n}}\right|^{2}\right\rangle
$$

In this case the $g$-factor may be calculated from the following relation:

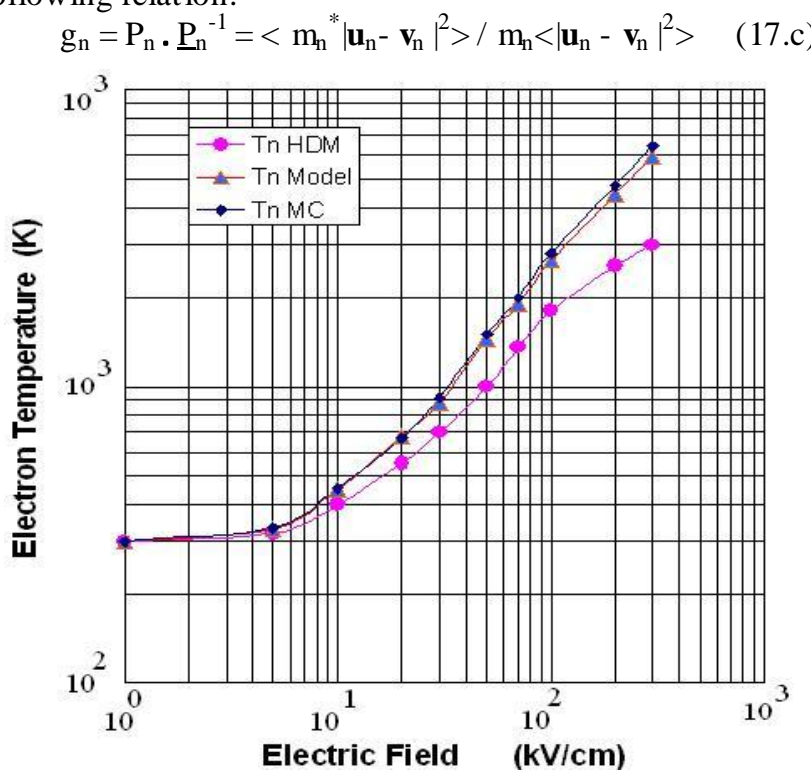

Figure 1. Electron temperature according to MC[29], classic HDM and our model

Figure 1 depicts the variation of $T_{n}$ according to $\mathrm{MC}$ (with a nonparabolicity factor $0.5 \mathrm{eV}^{-1}$ ) and the classic hydrodynamic model (14-b) and to our definition (17-b). Note that assuming $g_{n} \approx 1$ brings an error less than $3 \%$ in $T_{n}$, for electric fields up to $300 \mathrm{kV} / \mathrm{cm}$. This error is less drastic than the error encountered when neglecting the convective part in the calculation of the electron energy. In fact, most of the comme rcial simulators neglect totally the convection part of the carrier mean energy. If we adopt the assumption of unity $g_{n}$, we can drop the $g_{n}$ factor from the above system of hydrodynamic equations while keeping the non-parabolicity effects, via the average mass term. In this case, the scalar temperature definition reduces to:

$$
3 / 2 \mathrm{k}_{\mathrm{B}} \mathrm{T}_{\mathrm{n}} \approx 1 / 2 \mathrm{~m}_{\mathrm{n}}\left\langle\left|\mathbf{u}_{\mathrm{n}}-\mathbf{v}_{\mathrm{n}}\right|^{2}\right\rangle
$$

This approximation means that we assume the electrokinetic pressure tensor $\boldsymbol{P}_{n}=n k_{B} \boldsymbol{T}_{n}$, and may be considered as a closure condition for the set of HDEs. However, if later full-band MC (FBMC) showed a significant deviation of the g-factor from unity at very high fields, it could be easily retained in the proposed HDM to get more accurate results.

\section{Energy-Dependent Physical Parameters}

The accurate modelling of transport parameters, as the carrier drift mobility and impact ionization rate at high energies, is vital in any transport model. In the DDM, these parameters were usually expressed as functions of the local electric field [54-55]. Nevertheless, we know that these parameters express physical mechanisms involving energy exchange and should be expressed as functions of the carrier energy, rather than the local electric field. Fortunately, the solution of the HDM produces such information about the carrier energy distribution and permits to express these parameters in a physical manner, along the semiconductor device. In the following subsections, we discuss the modelling of the energy-dependent physical parameters in the bulk of silicon devices.

\subsection{Carrier Drift Mobility}

\subsubsection{Carrier Drift Mobility in the Bulk}

In the drift-d iffusion model, the high-field drift mobility in the bulk of a semiconductor is usually expressed by the Caughey-Thomas model, in its scaled form[56]:

$$
\mu_{n o}(\zeta)=\frac{\mu_{n o}}{\left[1+\left(\frac{\mu_{n o} \zeta}{v_{n}^{s a t}}\right)^{2}\right]^{1 / 2}}
$$

where $\mu_{n o}=\mu_{n o}\left(D O P, T_{L}, n, p\right)$ is the low-field mobility of electrons, which is related to doping, temperature and injection level, and may be expressed by a suitable model, e.g., Dorkel-Leturcq[57] or Klassen[58] models. Also $v_{n}{ }^{\text {sat }}$ is the electron saturation velocity and may be expressed by the model of Canali et al.[59]:

$$
\mathrm{v}_{\mathbf{n}}{ }^{\text {sat }}=2.4 \times 10^{7} /\left[1+0.8 \exp \left(\mathrm{T}_{\mathrm{L}} / 600\right)\right] \mathrm{cm} / \mathrm{s}
$$

However, in the hydrodynamic model where the carrier average energy can be determined, the high-field mobility can be expressed in terms of the carrier mean energy $\omega_{n, p}$. The energy-dependent drift mobility relation may be written in the following form[60]: 


$$
\mu_{n}^{-1}=\mu_{n o}^{-1}\left[1+\frac{\mu_{n o}}{e\left[v_{n}^{s a t}\right]^{2}}\left(\frac{\omega_{n}-\omega_{o}}{\tau_{\omega n}}\right)\right]
$$

This model resembles the model of Hänsch and Mattausch[61], but we consider an energy-dependent relaxation time and we make no a priori assumption about the distribution function in its derivation[62]. Figure 2(a) shows the variation of electron drift mobility as a function of electron temperature, according to our model and according to the model of Hänsch-Mattausch. In our model $\tau_{\omega n}$ is taken from MC simulation, as shown in Figure 4(a). In order to compare our model with the field-dependent phenomenological relations, one can substitute equation (18-b) into the set of HDE's in steady state homogeneous case to obtain:

$$
\left(\mu_{\mathrm{no}} / \mu_{\mathrm{n}}\right)=\left(\mu_{\mathrm{n}} / \mu_{\mathrm{no}}\right)\left(\mu_{\mathrm{no}} \zeta / \mathrm{v}_{\mathbf{n}}{ }^{\mathrm{sat}}\right)^{2}+1
$$

The solution of this quadratic equation (in $\mu_{n}$ ) is given by the following relation:

$$
\mu_{n}\left(\zeta_{/}\right)=\frac{2 \mu_{n o}}{1+\sqrt{1+\left[2 \mu_{n o} \zeta_{/} / v_{n}^{\text {sat }}\right]^{2}}}
$$

where we replaced $\zeta$ by the parallel field component $\zeta_{/ /}$, which is responsible of carrier heating, to satisfy the Thornber scaling theory [63]. As shown in Figure 2(b), this model coincides with the conventional field-dependent Caughey-Thomas mobility model[56] as well as the measured drift mobility, according to Canali et al[59]. The change of saturation velocity with lattice temperature may be also modelled as follows:

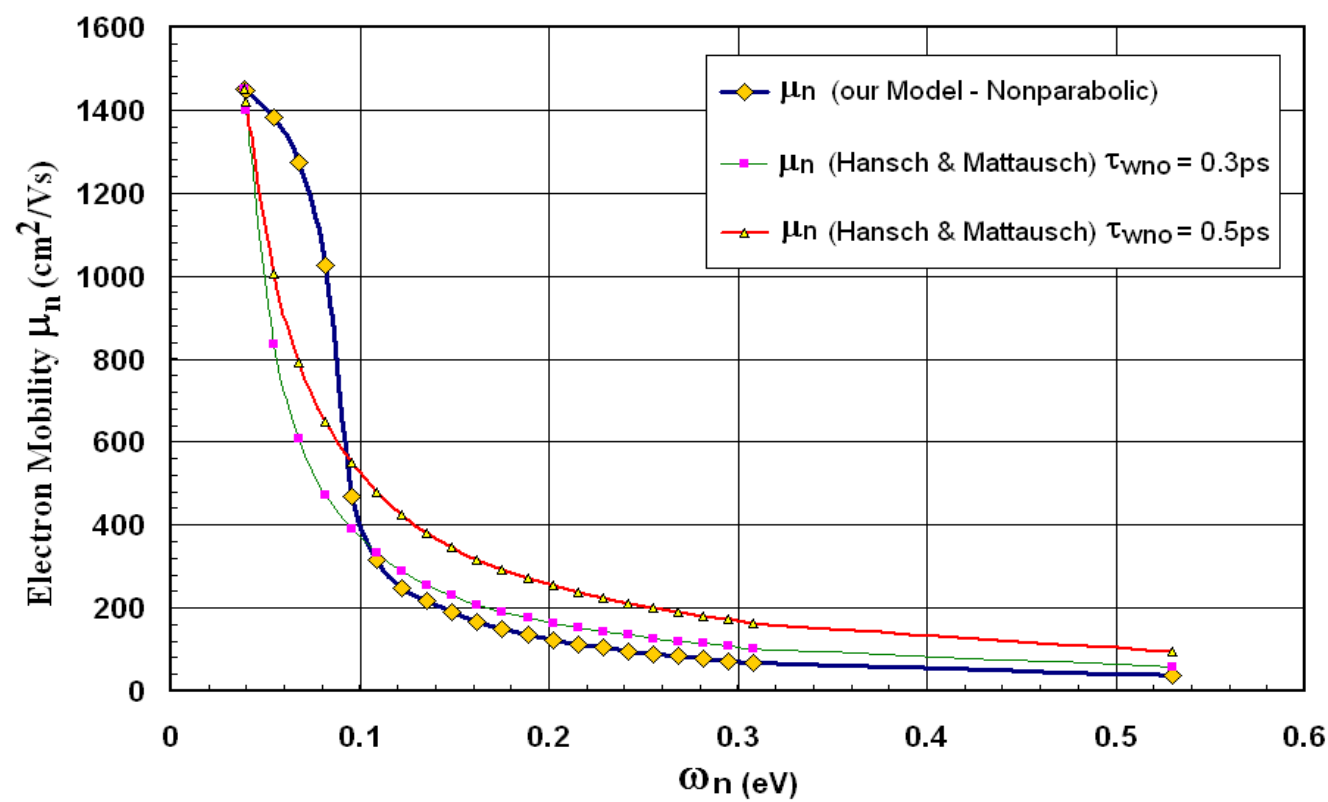

Figure 2(a). Electron drift mobility in Si at 300K versus elect ron temperat ure, according to the model of Hänsch-Matt ausch with different values of $\tau_{\oplus n o}[43]$ and according to our model

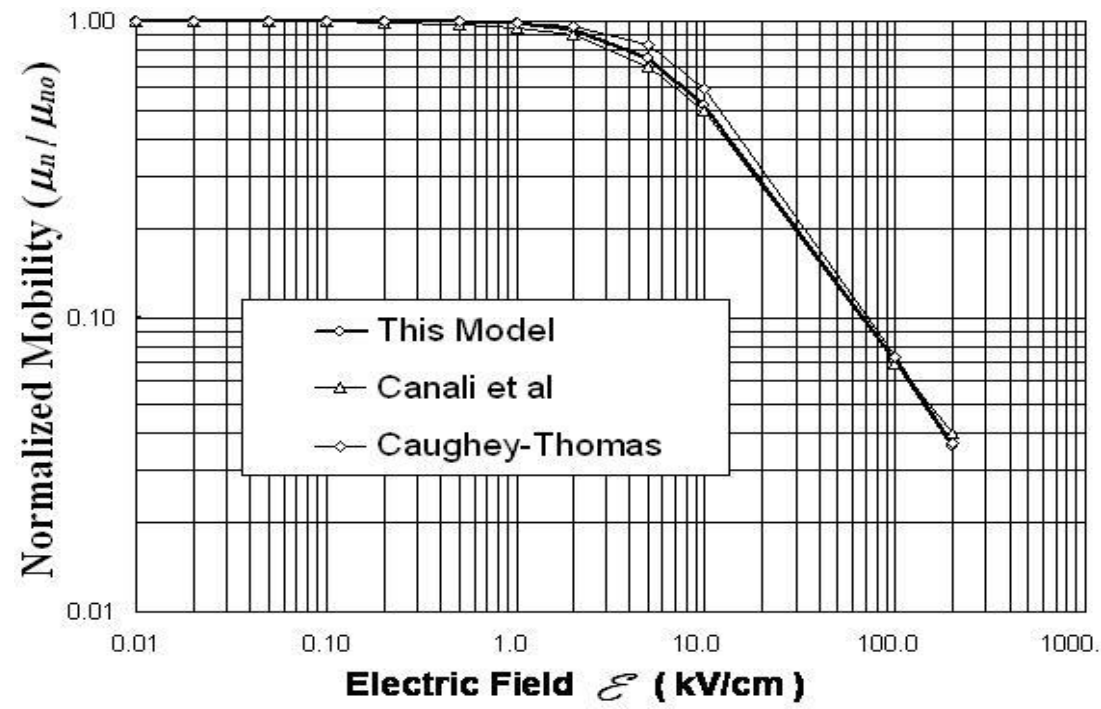

Figure 2(b). Electron drift mobility in $\mathrm{Si}$ at 300K, according to Canali et al[56], Caughey-Thomas model[52] and our model 


\subsubsection{Carrier Drift Mobility near the Surface}

The drift mobility near the surface of a semiconductor is greatly reduced because of the additional scattering mechanis ms due to the rupture of crystal periodicity, the surface phonons, the surface roughness and the eventual presence interface states. In addition, the presence of confined electrons into a thin layer near the surface of MOSFETs (the inversion layer) gives rise to quantization effects (subbands). As suggested by Sabins and Clemens [64], the reduction in the effective surface mobility may be expressed as a unique function of the effective vertical fie ld $\zeta \perp$ The most famous model of the surface mobility in MOSFET devices has been, for long time, the Yamaguchi model[65]:

$$
\mu_{c}\left(\zeta_{\perp}\right)=\frac{\mu_{b}}{1+\sqrt{1+\left(\zeta_{\perp} / \zeta_{c}\right)^{2}}}
$$

where $\zeta_{\perp}=|\zeta \mathrm{X} \boldsymbol{J} n| /|\boldsymbol{J} n|, \zeta_{c}$ is a constant $\left(1.49 \times 10^{4} \mathrm{~V} / \mathrm{cm}\right.$ for electrons and $1.87 \times 10^{4} \mathrm{~V} / \mathrm{cm}$ for holes in $\mathrm{Si}$ ), and $\mu_{b}=$ $\mu_{n}\left(\right.$ Dop, $\left.T_{L}, n, p, \zeta_{/ /}\right)$is the bulk drift mobility. According to Hänsch-Mattausch model[61], the drift mobility along the semiconductor surface is given by the following relation:

$$
\mu_{c}=\frac{2 \mu_{s}}{1+\sqrt{1+\left[2 \mu_{s} \zeta_{/ /} / v_{n}^{\text {sat }}\right]^{2}}}
$$

which resembles equation (19-b) but with the low-field mobility $\mu_{n o}$ replaced with the reduced surface mobility $\mu_{s}$, which is given by:

$$
\mu_{s}\left(\zeta_{\perp}\right)=\frac{\mu_{o}}{1+\gamma_{s} \zeta_{\perp}^{2}}
$$

where $\mu_{o}$ and $\gamma_{s}$ are constants. In fact, the channel mobility can be modeled by the Schwarz empirical formula, when we neglect the effect of generated surface states [66]:

$$
\mu_{n}\left(\zeta_{\perp}\right)=\frac{670}{1+\left(1.49 \times 10^{6} \zeta_{\perp}\right)^{1.66}} \mathrm{~cm}^{2} / \mathrm{V} . \mathrm{s}
$$

Another model, due to Sch warz and Russek[67], considers the channel broadening quantum effects and expresses the channel drift mobility as follows:

$$
\mu_{c}\left(\zeta_{\perp}\right)=\frac{\mu_{b}}{1+\alpha_{s} \mu_{b} \zeta_{\perp} /\left(1+\beta_{s} \zeta_{\perp}^{2 / 3}\right)}
$$

where $\alpha_{s}$ and $\beta_{s}$ are constants. The effective vertical field $\zeta \perp$ is sometimes related to the surface potential and may be approximated by the following relation, for MOSFETs wih $\mathrm{n}+$ polysilicon gate under strong inversion conditions [68]:

$$
\zeta_{\perp} \approx\left(\frac{\varepsilon_{o x}}{\varepsilon_{s i}}\right)\left(\frac{V_{G}+V_{T}}{2 x_{o x}}\right) \approx \frac{V_{G}+V_{T}}{6 x_{o x}}
$$

where $\varepsilon_{s i}$ and $\varepsilon_{o x}$ are the dielectric constants of silicon and gate oxide, $V_{G}, V_{T}$ and $x_{o x}$ are the MOSFET gate voltage, threshold voltage and gate oxide thickness, respectively.

A more elaborate and universal field-dependent mobility model, which reproduces the measured mobility data of
Takagi et al.[69] at the surface of semiconductor devices is given by Darwish et al.[70]. According to Darwish's model, the total carrier mobility is expressed by an equation similar to the scaled Hansch-Murrata model (21.a), with $\mu_{s}$ is given by:

$$
\mu_{s}^{-1}\left(\zeta_{\perp}\right)=\mu_{b}^{-1}+\mu_{s r}^{-1}+\mu_{a c}^{-1}
$$

Here $\mu_{b}$ is again the bulk mobility, $\mu_{s}$ is the carrier mobility limited by surface roughness and $\mu_{a c}$ is the carrier mobility limited by surface acoustic phonons. In the later model, the bulk mobility, $\mu_{b}$, is expressed by the klassen model[58], the surface acoustic, $\mu_{a c}$, follows the Lombardi model[71]:

$$
\mu_{a c}\left(\zeta_{\perp}\right)=\frac{B}{\zeta_{\perp}}+\frac{C}{T} \cdot \frac{D o p}{\zeta_{\perp}^{1 / 3}}
$$

where $B$ and $C$ are constants $\left(\mathrm{B}=3.61 \times 10^{7} \mathrm{~cm} / \mathrm{s}\right.$ for electrons and $1.51 \times 10^{7} \mathrm{~cm} / \mathrm{s}$ for holes, $\mathrm{C}=1.7 \times 10^{4} \mathrm{~cm}^{14 / 3} / \mathrm{V}^{2 / 3} \mathrm{~s} . \mathrm{K}$ for electrons and $4.18 \times 10^{3} \mathrm{~cm}^{14 / 3} / \mathrm{V}^{2 / 3} \mathrm{~s} . \mathrm{K}$ for holes) and the surface roughness limited mobility, $\mu_{s r}$, is given by:

$$
\mu_{s r}\left(\zeta_{\perp}\right)=\frac{\delta}{\zeta_{\perp}^{\gamma}}
$$

where $\delta$ and $\gamma$ are constants, depending on the technology $\left(\delta \approx 3.58 \times 10^{18} \mathrm{~V} / \mathrm{cm}\right.$ for electrons and $4.1 \times 10^{15} \mathrm{~V} / \mathrm{cm}$ for holes, $\gamma \approx 2.0-2.9$ )

\subsection{Impact Ionization Rate}

The generation by impact ionization is one of the most important mechanisms limiting the performance of semiconductor devices in general and the submicron MOSFETs in particular. In fact, the electron-hole pair generation by impact ionization, $G_{i i}$, influences so many characteristics of semiconductor devices such as the breakdown voltage and hot carrier injection currents. The impact ionization mechanis $\mathrm{m}$ was identified, about 50 years ago, by McKay[72]. Since then, the literature of the impact ionization mechanis $m$ has been extensive, e.g. 73-108], but only a few reviews are available. The generation rate by impact ionization $G_{i i}$ has been always expressed in terms of Townsend coefficients (or ionization rates of electrons and holes), $\alpha_{n}, \alpha_{n}$, as follows:

$$
\mathrm{G}_{\mathrm{i}}=\alpha_{\mathrm{n}} \mathrm{n} \mathrm{v}_{\mathrm{n}}+\alpha_{\mathrm{p}} \mathrm{p} \mathrm{v}_{\mathrm{p}}=\alpha_{\mathrm{n}}\left|\mathrm{J}_{\mathrm{n}} / \mathrm{e}\right|+\alpha_{\mathrm{p}}\left|\mathrm{J}_{\mathrm{p}} / \mathrm{e}\right|
$$

In the DDM, the impact ionization rates are conventionally expressed as functions of the local electric field. For instance, Wolff[73] used the first two terms of the Legendre expansion of the momentum distribution function, and derived the following electron impact ionization rate:

$$
\alpha_{n}(\zeta)=\alpha_{\text {no }} \exp \left[-\mathrm{C} / \zeta^{2}\right]
$$

where $\alpha_{n o}$ and $C$ are constants. According to Baraff[74], this procedure is only justifiab le at very high electric fields where electron-phonon collisions are frequent and the momentum distribution function is isotropic. Also, Keldish [75] solved analytically the BTE at high energy assuming parabolic band structure and calculated the impact ionization rate using the symmetric part of the distribution function. The Keldish solution attracted the attention because it tends to the Wolff 
relation $\left(\exp \left[-C / \zeta^{2}\right]\right)$ at high fields and tends to a simple exponential $\exp \left[-C^{\prime} / \mid \zeta\right]$ (where $C^{\prime}$ is another constant) at moderate fields, which coincides with the experiment. In fact, the measurements of Chynoweth[74], Lee et al[77], Van Overstraten and Deman[78], Kotani and Kawazu[79], Maes[80] and Takayangi[81] showed that the impact ionization rate at moderate fields follow a simple exponential function. According to Grant's model[82], the electron impact ionization rate follows the following exponential relation:

$$
\alpha_{n}(\zeta)=\alpha_{n o} \exp \left[-\zeta_{c n} /|\zeta|\right]
$$

where $\zeta_{c n}$ is the critical field at the onset of impact ionization. Shockley tried to find out an explanation of this experimental exponential relation in his study of $p-n$ junctions breakdown[83]. According to Shockley, the impact ionization mechanis $\mathrm{m}$ is mainly due to streaming of "lucky" electrons which can escape from collisions, and hence the ionization rate of such electrons can be expressed as follows:

$$
\alpha_{\mathrm{n}}(\zeta)=\alpha_{\mathrm{no}} \exp \left[-\mathrm{d} / \lambda_{\mathrm{op}}\right]
$$

where $d=\left(E_{n}{ }^{\text {th }} / e \zeta\right)$ is the minimum path an electron should travel to gain the threshold energy and $\lambda_{o p}$ is the mean free path between collisions with optical phonons. This model has been utilized to describe the breakdown phenomenon as well as the hot carrier injection and leakage currents in MOSFET devices in DDM-based simulation for long time[84-85]. Unfortunately, the parameters of the lucky-electron exponential model are dispersed among the different measurements of different authors in the literature [84]. In fact, the impact ionization phenomenon happens when drifting electrons have enough energy to ionize the valence electrons of lattice atoms. As the electron energy depends on the whole path of the electron under the effect of both the electric field and collisions, the local electric field value cannot directly reflect the real value of its energy. Thus, the local electric field dependent models of the impact ionization are not physically adequate, and their success in certain classic cases is due to their adjustable parameters. However, with the advent of the hydrodynamic model for semiconductors, where the carrier energy distribution is available, several authors tried to express the impact ionization rates in terms of the carrier mean energy or temperature.

\subsubsection{Schöll-Quade Impact Ionization Model}

The model of Schöll and Quade[87-88] for imact ionization rate is based on the heated Maxwellian approximation for the symmetric part of the distribution function. According to this model, the impact ionization rate of electrons is given by:

$$
\alpha_{n}\left(\omega_{n}\right)=\frac{1}{v_{n}^{\text {sat }} \tau_{o} \sqrt{\pi}}\left[\sqrt{\varpi_{n}} \cdot \exp \left(-\frac{1}{\varpi_{n}}\right)-\sqrt{\pi} \cdot \operatorname{erfc}\left(\frac{1}{\sqrt{\varpi_{n}}}\right)\right](25)
$$

where $\varpi_{n}=k_{B} T_{n} / E_{n}^{\text {th }}, E_{n}^{\text {th }}$ is the electron ionization threshold energy, $\tau_{o}$ is a constant $\left(\tau_{o}=\varepsilon_{o}{ }^{2} \hbar^{3} \pi^{3} / 3 e^{4} m_{n}{ }^{*} \approx 0.0126 f s\right)$ and $\operatorname{erfc}(x)$ is the complementary error function. Note that this model considers a parabolic energy band (constant effective mass $m_{n}{ }^{*}$ ). In order to get adequate results at moderate fields, both $E_{n}{ }^{\text {th }}$ and $\tau_{o}$ are usually considered as adjustable parameters, with values which are extremely far from their physical meaning. For instance, Souissi et al[89] considered $E_{n}^{\text {th }} \approx 4 \mathrm{eV}$ and $\tau_{o} \approx 0.14 f s$, in order to fit this model with experiment.

\subsubsection{Baccarani \& Stork Impact Ionization Model}

The model of Baccarani and Stork belongs to the category of semi-empirical models, which are based on the Shockley lucky-e lectron model. According to Baccarani and Stork[90], the rate of impact ionization is given by:

$$
\alpha_{n}\left(\omega_{n}\right)=c \lambda_{w} \cdot \exp \left(-\frac{3 \lambda_{w}}{5 \lambda_{o p}} \frac{E_{n}^{t h}}{\left(\omega_{n}-\omega_{o}\right)}\right)
$$

where $c, \lambda_{\omega}, \lambda_{o p}$ and $E_{n}^{\text {th }}$ are constants. According to Baccarani and Stork, the ionization threshold energy $E_{n}^{\text {th }}$ $=1.1 \mathrm{eV}$, the energy re laxation length $\lambda_{\omega}=(5 / 3) v_{n}^{\text {sat }} \tau_{\omega n o}=500 \AA$, the mean free path between opical phono collisions $\lambda_{o p}=65 \AA$ and the adjustable parameter $c$ is equal to $2 \times 10^{12} \mathrm{~cm}^{-2}$. However, Souissi et al.[89] used the model of Baccarani and Stork in their investigation about impact ionization in bipolar transistors and found that the best fit with measured values implies $E_{n}{ }^{t h}=5.077 \mathrm{eV}$. It has been shown in the latter work that both the Schöll-Quade and Baccarani-Stork models may be used to predict the multiplication factor $\left(M=\int \alpha_{n} d x\right)$ across the base-collector zone with reasonable error, only over a limited range of applied bias.

\subsubsection{Non-Maxwellian Distribution Function-based Impact Ionization Models}

In order to get rid of the disadvantages of the above mentioned models, some authors tried to calculate the impact ionization rate as a function of carrier energy, starting from its microscopic definition, on the basis of non-Maxwellian distribution functions [91-98]. For instance, Matsuzawa, Komahara and Wada[90] suggested the following form for the hot carrier tail of electrons distribution function:

$$
f_{n}\left(E_{n}\right)=C_{1} \exp \left[-1 / 2\left(E_{n} / k_{B} T_{n}\right)^{3 / 2}\right]
$$

where $C_{l}$ is a constant. Also, Sonoda et al.[96] and later Grasser et al[97] assumed the following two-components distribution, to account for both cold and hot carriers populations in nanoscale MOSFET devices:

$\mathrm{f}_{\mathrm{n}}\left(\mathrm{E}_{\mathrm{n}}\right)=\mathrm{C}_{1} \exp \left[-\left(\mathrm{E}_{\mathrm{n}} / \mathrm{E}_{\mathrm{ref}}\right)^{\mathrm{b}}\right]+\mathrm{C}_{2} \exp \left[-\left(\mathrm{E}_{\mathrm{n}} / \mathrm{k}_{\mathrm{B}} \mathrm{T}_{\mathrm{n}}\right)\right](27 . \mathrm{b})$ where $C_{1}, C_{2}, E_{r e f}$ and $b$ are adjustable parameters, which are used to tune the hot carrier distribution component, and the cold carrier (Maxwellian) component, in order to obtain reasonable results over a wide range of carrier energies. Therefore, when $C_{1}=0$, the distribution function becomes a heated maxwellian and when $C_{2}=0$ it tends to the tail distribution function. Using this four-parameters distribution function and assuming parabolic energy bands, Morris, Pass and Abebe introduced a simple formulation for the impact ionization rate on the bas is of Gauss-Laguerre appro ximation [98].

It should be noted that several efforts have been devoted for the development a nonlocal impact ionization model on 
the basis of the hot-electron subpopulation, e.g.,[99]-[100]. T-W.Tang and Nam have introduced a simp lification of such hot-carrier subpopulation models[101]. The impact ionization coefficient is then fitted to homogeneous MC simulation in the bulk of $\mathrm{Si}$ as follows:

$$
\alpha_{\mathrm{n}}\left(\omega_{\mathrm{n}}\right)=9.59 \times 10^{5} \exp \left[-\left(134 / \mathrm{E}_{\mathrm{ref}}\right)\right]
$$

for $\omega_{\mathrm{n}}>0.164 \mathrm{eV}=1.156 \times 10^{6} \mathrm{exp}\left[-\left(90 / \mathrm{E}_{\mathrm{ref}}\right)\right]$ els where $(28)$

\subsubsection{Advanced Impact Ionization Model}

Unfortunately, the success of the above mentioned energy-dependent models of impact ionization is limited to specific cases at small and moderate fields. In addition, these models make use of a number of adjustable parameters, whose fitting values have sometimes irrelevant physical meaning. We think that one source of errors is their lake to a link with the realistic band structure of the semiconductor material. The following phenomenological model accounts for the energy band structure and can be used, with no need to curve fitting of its physical parameters [102].

$$
\mathrm{G}_{\mathrm{ii}}=\left(\mathrm{n} / \tau_{\mathrm{in}}\right)+\left(\mathrm{p} / \tau_{\mathrm{ip}}\right)
$$

where $1 / \tau_{\text {in }}$ and $1 / \tau_{i p}$ are the electron and hole average ionisation rates. Starting from the scaled form of the impact ionization rate, accord ing to the Thornber theory [103]:

$$
\begin{gathered}
\alpha_{\mathrm{n}}(\zeta)=\left(1 / \mathrm{v}_{\mathrm{n}} \tau_{\mathrm{in}}\right) \\
=\left(\mathrm{e} \zeta^{\prime \prime} / \mathrm{E}_{\mathrm{n}}^{\mathrm{th}}\right) \exp \left[-\zeta_{\mathrm{c}} /\left\{\zeta^{\prime \prime}\left(1+\zeta^{\prime \prime} / \zeta_{\mathrm{p}}\right)+\zeta_{\mathrm{T}}\right\}\right.
\end{gathered}
$$
where $\zeta_{T}, \zeta_{p}$ and $\zeta_{c}=E_{n}^{t h} / e \lambda_{o p}$ are the critical fields at which the electrons surmount acoustic phonons, optical phonons and impact ionization scattering thresholds. Assuming that the ionization rate can be fully described by the kinetic moments (like, $v_{n}$ and $\omega_{n}$ ), one can write the energy conservation equation (10-c) in the following form in static field conditions:

$$
\begin{aligned}
& \text { en } \zeta \cdot v_{n}=-\left(\partial n \omega_{n} / \partial t\right)_{c o l} \approx n\left(\omega_{n}-\omega_{0}\right) / \tau_{\text {in }} \\
& -\left(\omega_{n}-\omega_{0}\right)\left(n-n_{0}\right) / \tau_{n}+n\left(\omega_{n}-\omega_{0}\right) / \tau_{\omega n}
\end{aligned}
$$

where the first term represent the generation by impact ionization, the second term represents the loss of electron energy due to recombination ( $\tau_{n}$ being the electrons lifetime and $n_{o}$ is the equilibrium electron density) and the third term represents the loss of electron energy due to collisions with optical phonons. The second term may be dropped from the above equation because $\tau_{\omega n}<<\tau_{n}$. Also the the first and third terms can be combined, with an effective energy relaxation time, which includes the effect of impact ionization at high energies. In fact, this effect is not significant, according to Schöll and Quade[88]. Substituting the product $e \zeta . v_{n}$ from (27-c) into (27-b), we get the following expression for the electron ionization rate at moderate and high fields:

$$
\alpha_{n}\left(\omega_{n}\right)=\frac{1}{\tau_{i n} v_{n}}=\frac{\left(\omega_{n}-\omega_{o}\right)}{\tau_{w n} v_{n} E_{n}^{t h}} \cdot \exp \left(-\frac{\eta_{n} E_{n}^{\text {th }}}{\left(\omega_{n}-\omega_{o}\right)}\left[\frac{E_{p}}{E_{p}+\left(\omega_{n}-\omega_{o}\right)}\right]\right) \text { (30.a) }
$$

where $\eta_{n}=\left(v_{n} \tau_{w n} / \lambda_{o p}\right), \lambda_{o p}$ is the electron mean free path between optical phonon collisions and $E_{p}$ is the mean optical phonon energy. At low and moderate fields, this model can be approximated to the following simple form:

$$
\alpha_{n}\left(\omega_{n}\right)=\alpha_{n \infty} \cdot \exp \left(-\frac{E_{n}^{I}}{\omega_{n}-\omega_{o}}\right)
$$

where $\alpha_{n o} \approx \omega_{n} /\left(E_{n}^{\text {th }} \tau_{w n} v_{n}{ }^{\text {sat }}\right)$ is the asymptotic value of impact ionization rate and $E_{n}{ }^{I} \approx E_{n}{ }^{\text {th }}\left(\tau_{w n} v_{n}{ }^{\text {sat }}\right) / \lambda_{o p}$ is the mean ionization threshold energy for electrons. However, at high fields the impact ionization rate is proportional to $\exp \left(-E_{n}{ }^{l} \cdot E_{p}\right.$ $\left./ \omega_{n}{ }^{2}\right)$. Note that we consider $\tau_{w n}=\tau_{w n}\left(\omega_{n}\right)$ and $\lambda_{o p}=\lambda_{o p}\left(\omega_{n}\right)$. So, even at low fields the exponential realation $\exp \left(-E_{n}{ }^{l} /\left(\omega_{n}-\omega_{o}\right)\right)$ does not imply a Maxwellian distribution because $E_{n}{ }^{I}$ is energy dependent. The energy dependence of the energy relaxation time, which implicitly includes the energy band structure effects, can be obtained fro m MC simulation or by measurement as indicated in the next section IV-C. Unlike classical models, the hard threshold energy $\left(E_{n}^{\text {th }}\right)$ is replaced with a soft (energy-dependent) mean threshold energy $\left(E_{n}{ }^{I}\right)$ in our model. This coincides with Bude and Hess conclusions about the impact ionization soft threshold [102]. The calculation of $\lambda_{o p}$ as a function of the electron energy may be carried out by MC simulation in the bulk, using the relation[105]:

$$
\lambda_{\text {op }}\left(\omega_{\mathrm{n}}\right)=\left\langle\mathrm{u}_{\mathrm{n}}(\mathrm{k}) / \Gamma_{\mathrm{n}}(\mathrm{k})\right\rangle
$$

where $\Gamma_{n}(k)$ is the total scattering rate by optical phonons. It may be also estimated from the so-called lucky-electron exponential model LE-EM [86]. In our analysis we used the following phenomenological relation, which is based on Crowell and Sze model[106], at the carrier te mperature:

$$
\lambda_{\text {op }}\left(\omega_{n}\right)=\lambda_{o} \tanh \left[E_{p} / 2 k_{B}\left(T_{n}-T_{L}\right)\right.
$$

where $\lambda_{0}$ is mean free path between collisions at low fields (when $T_{n} \approx T_{L}$ ). Figs. 3(a) and (b) depict the variation of $\lambda_{o p}$ and $\alpha_{n}$ in $\mathrm{Si}$, according to the above relations. In our calculations, we took $E_{p}=60 \mathrm{meV}$ and $\lambda_{0}=1000 \AA$ and exponentially extrapolated the available MC data of $\tau_{w n}\left(\omega_{n}\right)$, as shown in figure 4(a). For the matter of comparison, we plotted $\alpha_{n}$ according to Baccarani-Stork and Scholl-Quade models, beside our model. The Scholl-Quad model produces exceesively high rates when the physical value of $\tau_{o}$ is taken into account. So, we set $\tau_{o}$ equal to $12.6 \mathrm{fs}$, about 3 orders of magnitude greater than its physical value[86]. Note that the value of $\alpha_{n}$ in our scaled model is greater than all other models and continues to increase at high energies. In fact, the experimental values of $\alpha_{n}$ may exceed $10^{6} \mathrm{~cm}^{-1}$. For instance, the measurement of Kotani and Kawazu of $\alpha_{n}(\zeta)$ is usually fitted with $\alpha_{n o} \geq 10^{6} \mathrm{~cm}^{-1}[79]$. Also, the homogeneous MC simulation[105], indicates that the impact ionization pre-exponential is almost $10^{6} \mathrm{~cm}^{-1}$ at high energies, as depicted by equation (26). It worth noting that the impact ionization rate at high energies (in the saturation region) is greatly influenced by the roll-off of the enrgy rela xation time, while $E_{n}{ }^{\text {th }}$ and $\lambda_{o p}\left(\omega_{n}\right)$ have a relative influence at low and mediumenergies. This explains the failure of the previously suggested models, to properly estimate $\alpha_{n}$ at high energies, even with adjusting $E_{n}{ }^{\text {th }}$ and the pre-exponential factors with non physical values, while fixing both $\lambda_{o p}$ and $\tau_{w n}$. For the matter of verification, Figure $\mathbf{1 4}$ depicts the I-V characteristics of a reverse biased p-i-n diode near avalanche, with different transport approaches (the DDM, the classic HDM with Scholl-Quade model and our proposed HDM model). As shown, the I-V characteristics obtained by our 
model are softer and the breakdown voltage is smaller and more realistic than the classic HDM results.

\subsubsection{Energy Broadening of Impact Ionization Threshold}

The determination of the impact ionization threshold has been a subject of extensive research, both theoretically and experimentally[107]. In fact the measured values of the ionization threshold and those used in the theoretical models vary widely. As we pointed out in the last section, the mean ionization energy $E_{n}{ }^{I}$ in our model of impact ionization has a soft distribution around $E_{n}^{\text {th }}$. In order to evaluate the broadening of mean ionization energy, we define $\Delta E_{n}{ }^{I}$ as follows:

$$
\left(\omega_{\mathrm{n}}\right)=\left|\mathrm{E}_{\mathrm{n}}{ }^{\mathrm{I}}\left(\omega_{\mathrm{n}}\right)-\mathrm{E}_{\mathrm{n}}^{\text {th }}\right|=\mathrm{E}_{\mathrm{n}}{ }^{\text {th }}\left|1-\mathrm{v}_{\mathrm{n}} \tau_{\mathrm{wn}} / \lambda_{\mathrm{op}}\right|
$$

Figure 3(c) depicts the ionization energy broadening $\Delta E_{n}{ }^{I}$ as a function of mean electron energy. This broadening is mainly due to the variation of the energy rela xation time and the mean free path at high energies. Curiously, one may wonder if there is any reas on to relate this phenomenon with the broadening in energy levels due to finite lifetime of energy states between scattering events at high energy[108-109]. In fact, it has been shown experimentally in[110] that there exists a line broadening transition, representing the transition from phonon scattering dominated to impact ionization dominated transport not only in silicon but also in any material with a bandgap. In addition, some theoretical studies of the impact ionization on the basis of quantum transport showed that a fixed impact-ionization threshold does not exist, and the impact-ionization scattering rate is drastically enhanced around the semiclassical threshold by the intracollisional field effect[111]. Of course, the broadening factor may be better evaluated by precise MC simulation, taking into account the impact ionization mechanis m. However, with our simple model of $\lambda_{o p}\left(\omega_{n}\right)$ and the exponentially extrapolated $\tau_{w n}\left(\omega_{n}\right)$, we found that $\Delta E_{n}{ }^{I}$ is centralized around a minimum (almost zero) at $\omega_{\mathrm{n}} \approx 1.8 \mathrm{eV}$, which is interestingly equal to $3 / 2 E_{n}^{\text {th }}$. It should be noted here that we chose $E_{n}^{\text {th }}=E g \approx 1.2$ and chose $\lambda_{0}=1000 \AA$, such that the distribution of $\lambda_{o p}\left(\omega_{n}\right)$ is as close as possible from the experimental LE-EM curve. As shown in figure 3 (a), the two curves almost intersect at $\omega_{n}=E_{n}{ }^{t h}=1.2 \mathrm{eV}$.

\subsection{Carrier Energy Relaxation Time}

The choice of the energy relaxation time is crucial in the HD simulation because it influences both the static and dynamic characteristics of the device to be simulated. According to the hydrodynamic theory, the exact modelling of the carrier energy relaxation time (and all other relaxation times) should be expressed as function of all the carrier mo ments like $n, v_{n}$ and $\omega_{n}$, etc[112]. However, as we usually truncate the series of moments and satisfy ourselves with the few first mo ments, it is believed that much of the information about the energy rela xat ion time is re lated to the carrier mean energy and density[30]. According to Jacoboni and Reggiani[112], the energy relaxation time should decrease at high fields in a phenomenological way representing the tendency of the isotropic part of the distribution function to decay towards its equilibrium value. So, the later authors used the following equation, to evaluate the carrier energy relaxation time for warm electrons:

$$
\tau_{\mathrm{wn}}\left(\omega_{\mathrm{n}}\right)=\left(\omega_{\mathrm{n}}-\omega_{\mathrm{o}}\right) / \mathrm{e} \mathrm{v}_{\mathrm{d}} \zeta
$$

This relation, which is sometimes called the Seeger formula [114], reflects the energy continuity at steady state: $\partial \omega_{\mathrm{n}} / \partial \mathrm{t}=\mathrm{e} \mathrm{v}_{\mathrm{d}} \zeta+\left(\partial \omega_{\mathrm{n}} / \partial \mathrm{t}\right)_{\mathrm{col}}=\mathrm{e} \mathrm{v}_{\mathrm{d}} \zeta-\left(\omega_{\mathrm{n}}-\omega_{\mathrm{o}}\right) / \tau_{\mathrm{wn}}=0(33 . \mathrm{b})$

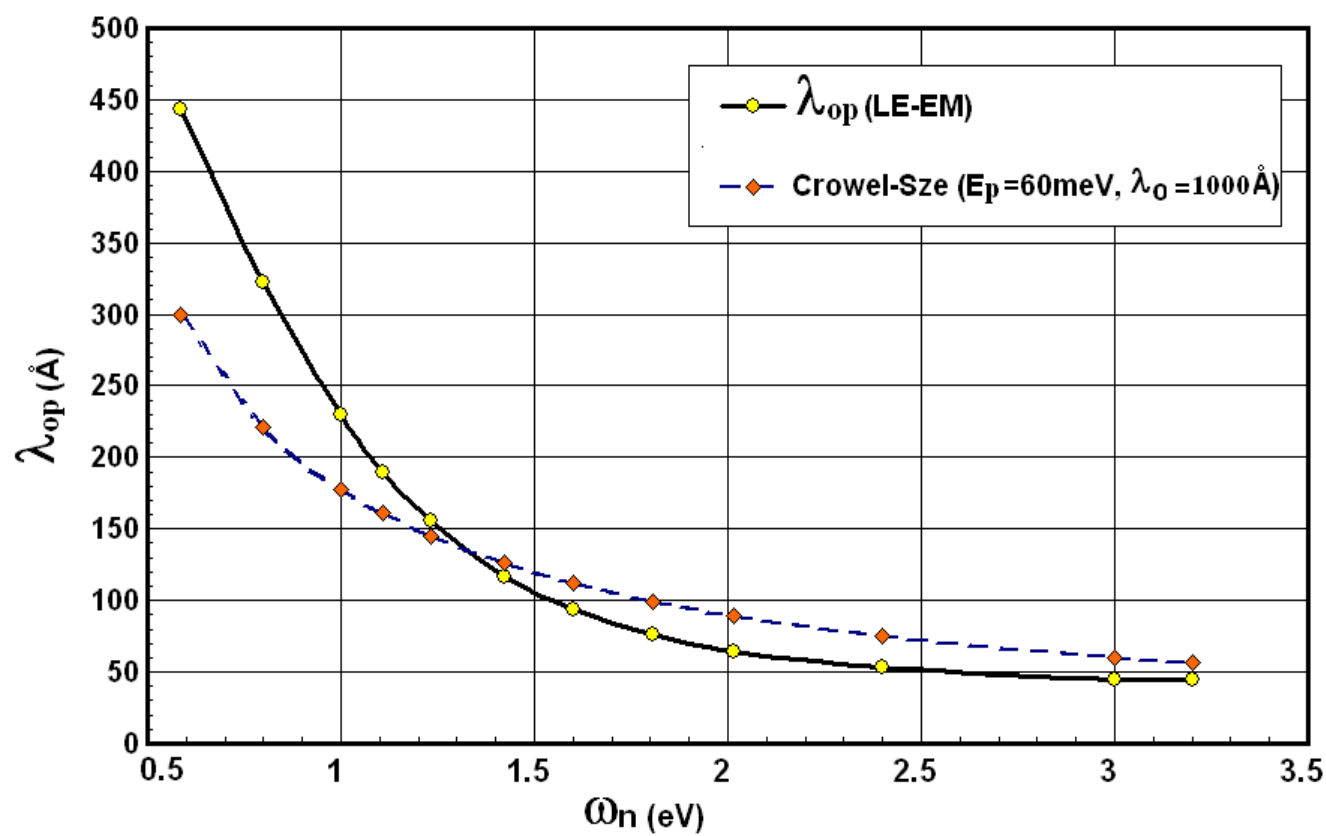

Figure 3(a). Electron mean free path between optical phonon collisions in $\mathrm{Si}$ at 300K, according to the lucky-electron exponential model (LE-EM), after[69], and according to the Crowel-Sze model[85] at the electronic temperat ure 


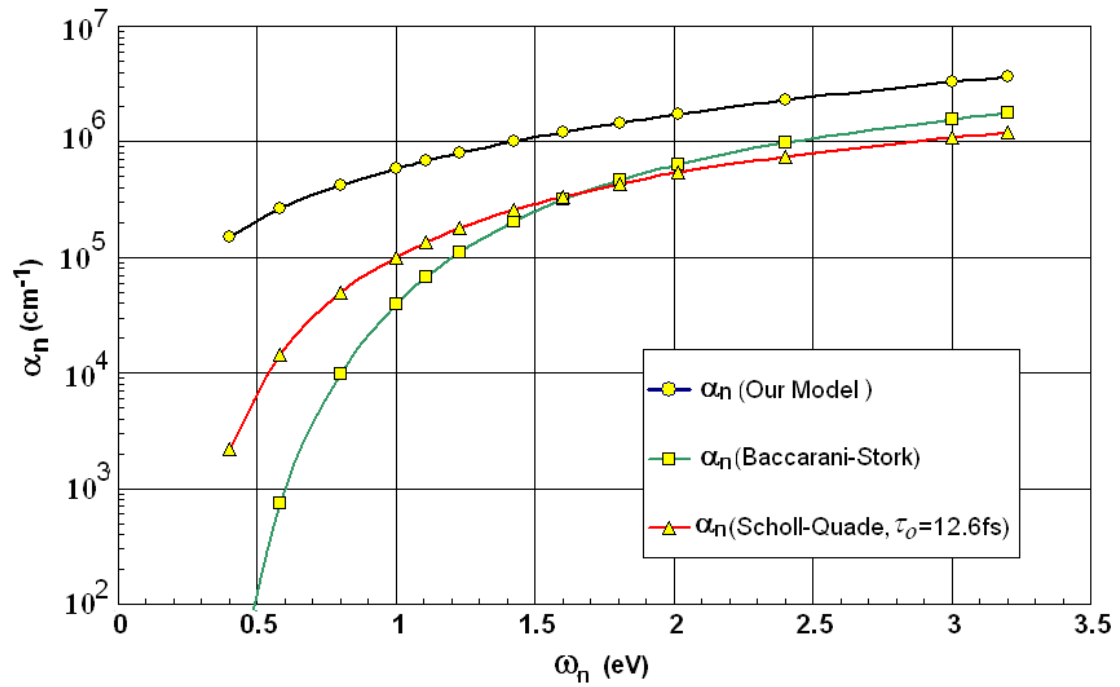

Figure 3(b). Electron impact ionization rate in $\mathrm{Si}$ at $300 \mathrm{~K}$, as a function of average electron energy, according to Baccarani-Stork model $\left(\lambda_{w}=500 \AA\right.$, $\left.\lambda_{\mathrm{op}}=65 \AA\right)$, Scholl-Quade model $\left(\tau_{\mathrm{o}}=12.6 \mathrm{fs}\right)$. In all models $\mathrm{E}_{\mathrm{n}}{ }^{\text {th }}=1.2 \mathrm{eV}$

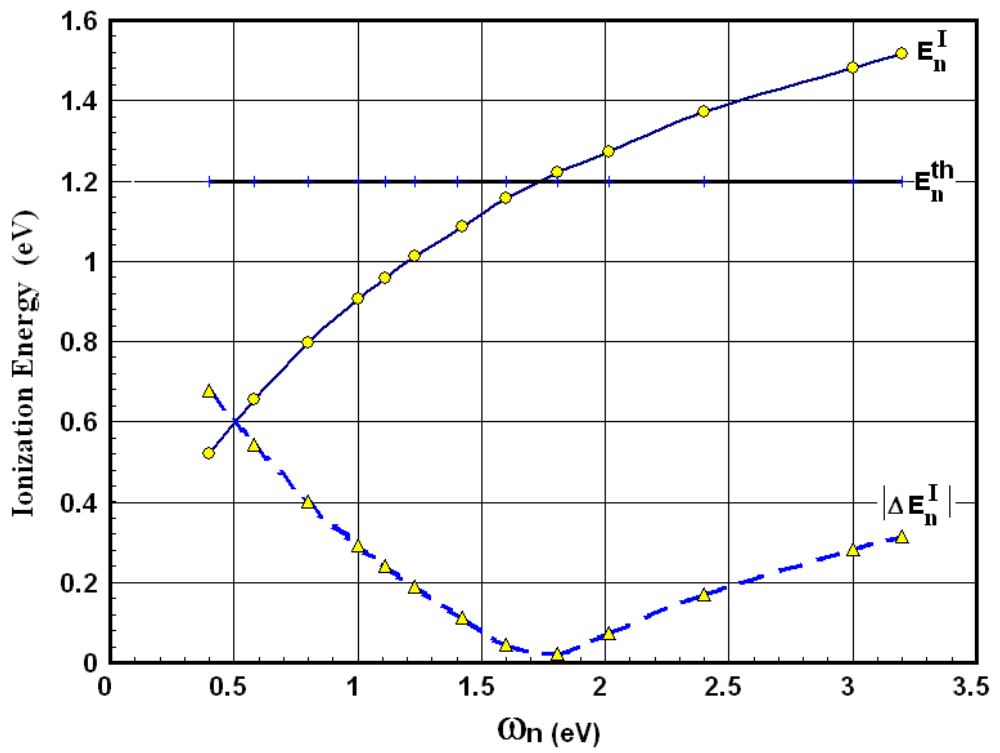

Figure 3(c). Average ionization energy in $\mathrm{Si}$ at $300 \mathrm{~K}$, according to our model model $\left(\lambda_{0}=1000 \mathrm{~A}, \mathrm{E}_{\mathrm{n}}{ }^{\text {th }}=1.2 \mathrm{eV}\right)$

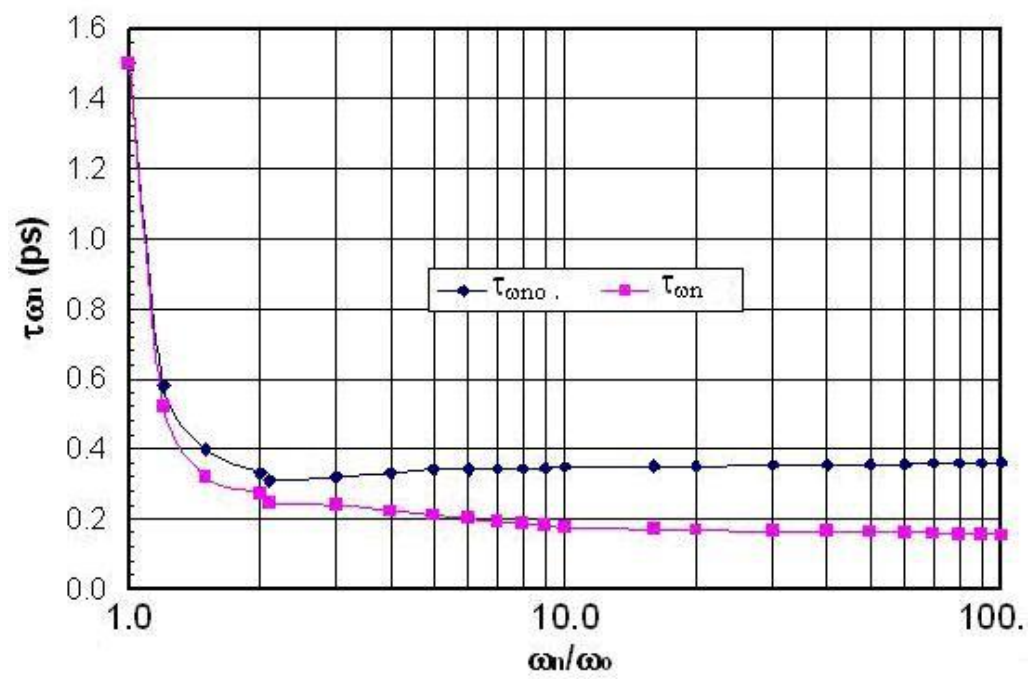

Figure 4(a). Variation of the electron energy relaxation time with electron average energy in $\mathrm{Si}$ at $300 \mathrm{~K}$, according to MC simulation. In the above curve, the energy bands are assumed parabolic. The lower curve takes the non-parabolicity of the conduction band into account (non-parabolicty factor $0.5 \mathrm{~V}^{-1}$ ) 


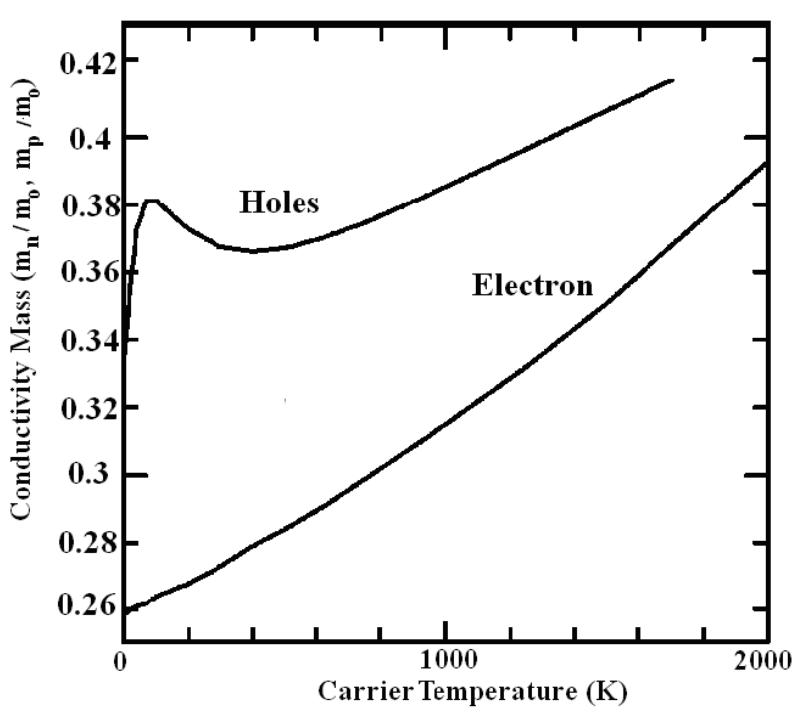

Figure 4(b). Experimental value of electron and holes conductivity mass, after Rife[95]

According to Seeger's static formula, the energy relaxation time can be determined by substituting the static field relations $v_{d}(\zeta)$ and $\omega_{n}(\zeta)$ from MC simulation[115]. Gonzalez et al. employed this relation to calculate the fitting parameters of their energy relaxation time model[116]. On the other hand, several authors considered the energy relaxation time as a constant when the electron distribution function is warm enough and the electron velocity tends to saturate. This is a good approximation when the band structure of the material is parabolic. For non-parabolic bands, the energy relaxation time continues to decrease when electron energy is further increased, as shown in Figure 4(a). In fact, it has been shown that the value of the energy relaxation time greatly influences the hydrodynamic simu lation results in deep submicron transistors [117]. Th is is due the fact that the intervalley phonons becomes more effective in dissipating the electron energy at high energies, which lead to decreasing the electron energy relaxation time, at higher energies. For accurate modelling of the energy relaxation time, we propose the following phenomenological relation between the electron average effective mass $m_{n}$ and $\tau_{w n}$, for energies above the equilibriu m value $\left(\omega_{n}>\omega_{o}\right)$ :

$$
\tau_{\mathrm{wn}}\left(\omega_{\mathrm{n}}\right)=\tau_{\mathrm{wno}}\left[\mathrm{m}_{\mathrm{no}} / \mathrm{m}_{\mathrm{n}}\left(\omega_{\mathrm{n}}\right)\right]
$$

where $m_{n o}$ is the density of states effective mass at the bottom of the conduction band (near equilibrium). This model is based on the physical fact that heavy electrons relax faster than lighter ones. In fact, the increased electron-phonon coupling at high fields (where the electron is said to be dressed in a cloud of phonons) leads to a modification of electron states and changes their energy (by addition of the self energy) and this increases the mass of electrons and makes their motion slower. Therefore, at high energies where the electron average mass increases as electron energy increases, the electron dissipates energy more efficiently and the energy relaxation time is shorter. According to the proposed relation, we only need the knowledge of the low-field value of the electron energy relaxation time $\tau_{\text {wno }}$ and the average mass as a function of electron energy $m_{n}\left(\omega_{n}\right)$ to obtain $\tau_{w n}\left(\omega_{n}\right)$. The low-field value of the average electron mass, $m_{n o}$, is equal to the conductivity effective mass at the conduction band edge, and can be measured using the optically-detected cyclotron resonance (ODCR). The ODCR method is more accurate than the classical CR and has been recently used to measure the effective mass of several semiconductor compounds [118]. According to the optical measurements of Rife[119] the conductivity effective mass at $300 \mathrm{~K} m_{\mathrm{no}}=0.275 \mathrm{~m}_{\mathrm{o}}$ and $m_{\mathrm{no}}=0.365 \mathrm{~m}_{\mathrm{o}}$ as shown in figure 4(b). The ultrafast laser based pump-probe techniques have been used to measure the energy relaxation as well as the mo mentum relaxation times in $\mathrm{Si}$. Using this technique, a time constant of $32 \pm 5$ fs associated with momentum relaxation and an electron - phonon energy relaxation time of $260 \pm 30$ fs have been extracted from the coherent-transient variations in (001) Si at 300K[120].

In order to verify our model, we used the MC simulation to calculate the average electron mass as a function of the average electron energy, and compared it with the measured ratio of $\left(m_{n o} / m_{n}\right)$, as shown in Figure 5. As shown in figure, the normalized value of the average inverse mass decreases as the electron energy increases. Then we calculated the energy relaxation time $\tau_{w n}\left(\omega_{n}\right)$, using the above phenomenological relation (above $\omega_{0}$, when electrons start to warm up). As shown in Figure 5, the discrepancy between the the energy relaxation time, according to MC and the calculated values according to our model lies within the measurement error $(\sim 10 \%)$ for a wide range of carrier temperatures (up to $2000 \mathrm{~K}$ and may be extrapolated to higher values). Alternatively, our model can be used to calculate the energy dependence of the average electron mass $m_{n}\left(\omega_{n}\right)$ from the energy dependence of the electron energy relaxation time $\tau_{w n}\left(\omega_{n}\right)$. The energy dependence of $\tau_{w n}$ of several semiconductors (e.g., AlGaAs/GaAs, $\mathrm{AlGaN} / \mathrm{AIN} / \mathrm{GaN}$ ) has been measured using the micro wave-noise method[121]. This method is based on the measurement of the noise temperature, which is equal to the electron temperature $T_{n}$ in the semiconductor, according to the following relation:

$$
\tau_{\mathrm{wn}}=3 / 2 \mathrm{k}_{\mathrm{B}}\left(\mathrm{T}_{\mathrm{n}}-\mathrm{T}_{\mathrm{L}}\right) / \mathrm{e} \mathrm{v}_{\mathrm{d}} \zeta
$$

where we neglect the convection part of the electron energy and consider $\omega_{n}=3 / 2 k_{B} T_{n}$. However, we have no experimental data yet about the energy relaxation time in $\mathrm{Si}$ (as a function of carrier temperature or energy) using this method. So, the proposed model which correlates $\tau_{w n}\left(\omega_{n}\right)$ to the already measurable $m_{n}\left(\omega_{n}\right)$ should be very useful in this case. 


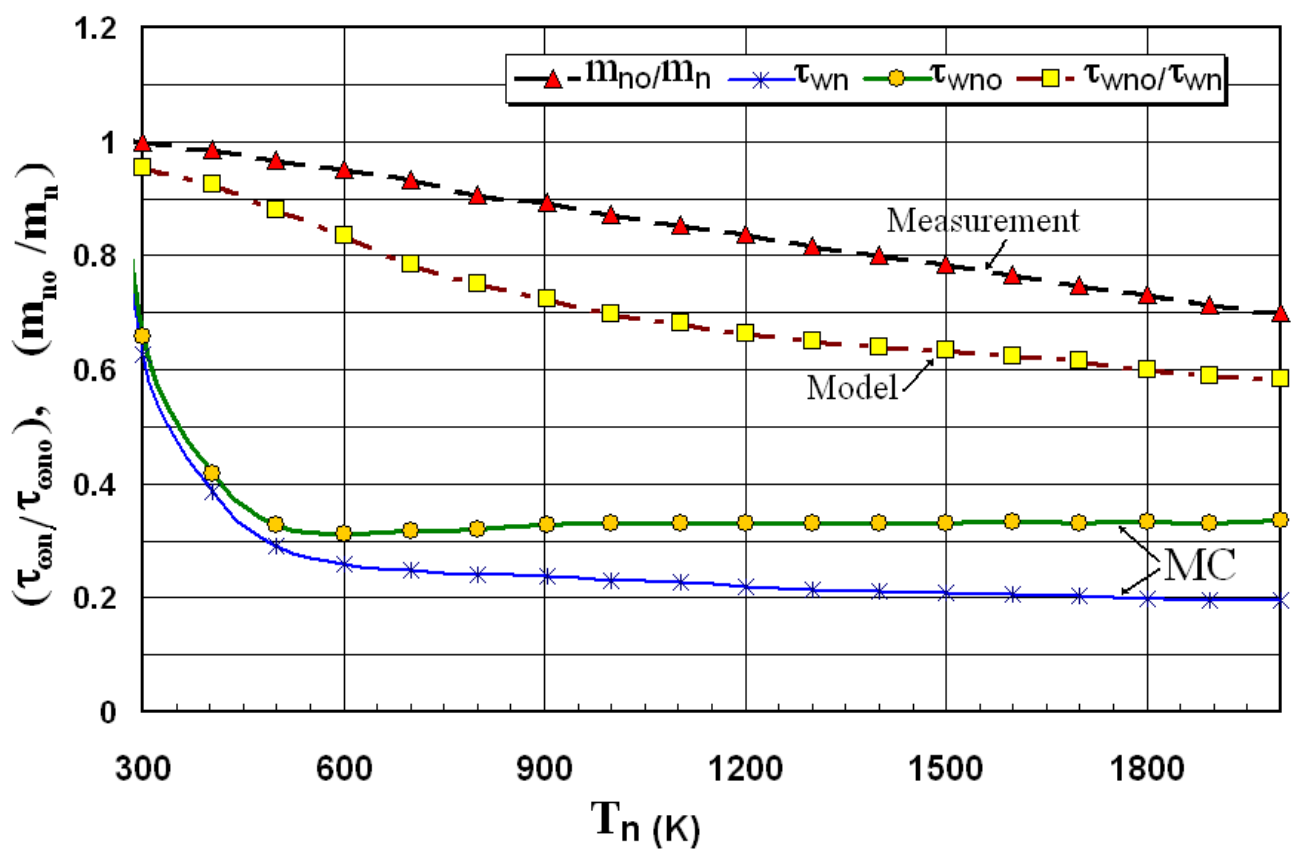

Figure 5. Comparison bet ween the normalized inverse mass $\left(\mathrm{m}_{\mathrm{no}} / \mathrm{m}_{\mathrm{n}}\right)$, according to optical measurement [52] and our model. The model is calculated from the normalized energy relaxation time $\left(\tau_{\omega n} / \tau_{\oplus n}\right)$ according to MC dat a, by our phenomenological relation (25)

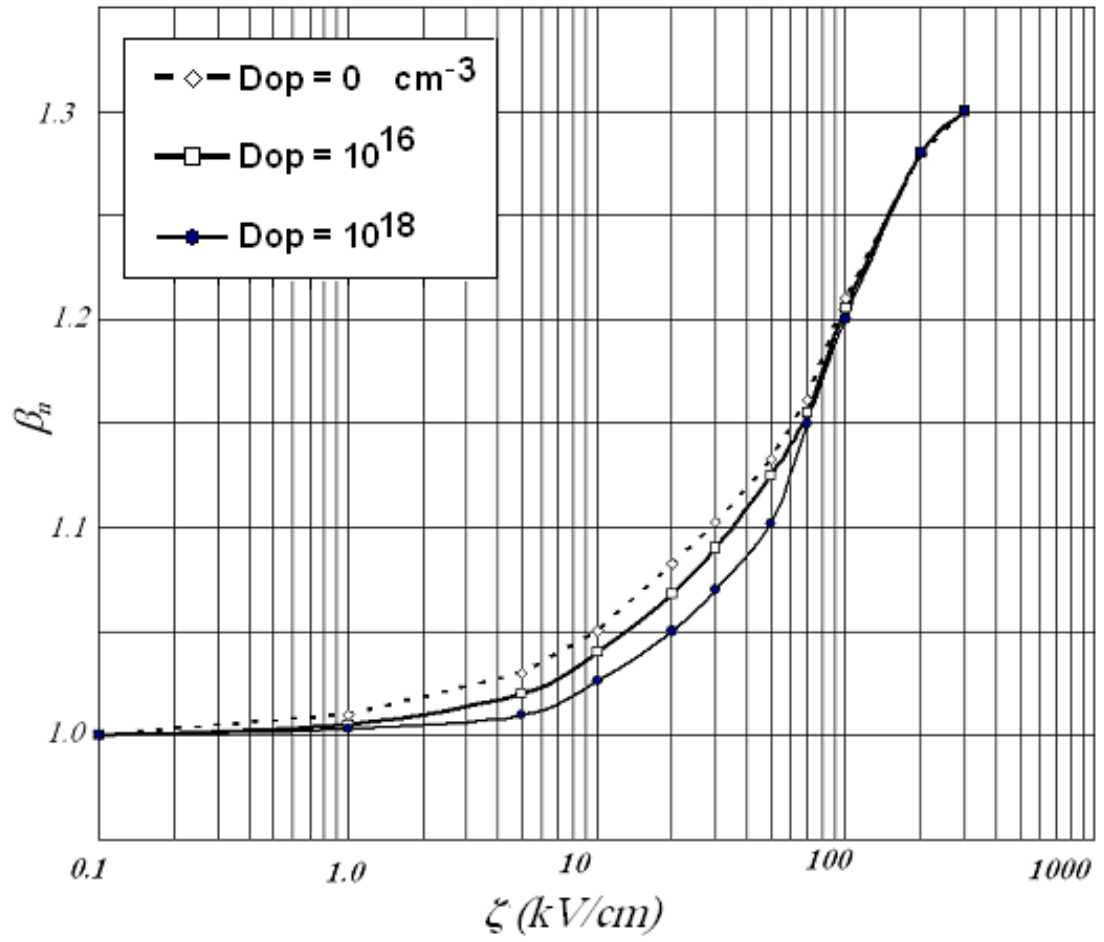

Figure 6(a). Heat flux beta fact or in $\mathrm{Si}$ at $300 \mathrm{~K}$, calculated from MC simulation, with non-parabolicty factor $0.5 \mathrm{~V}^{-1}$ 


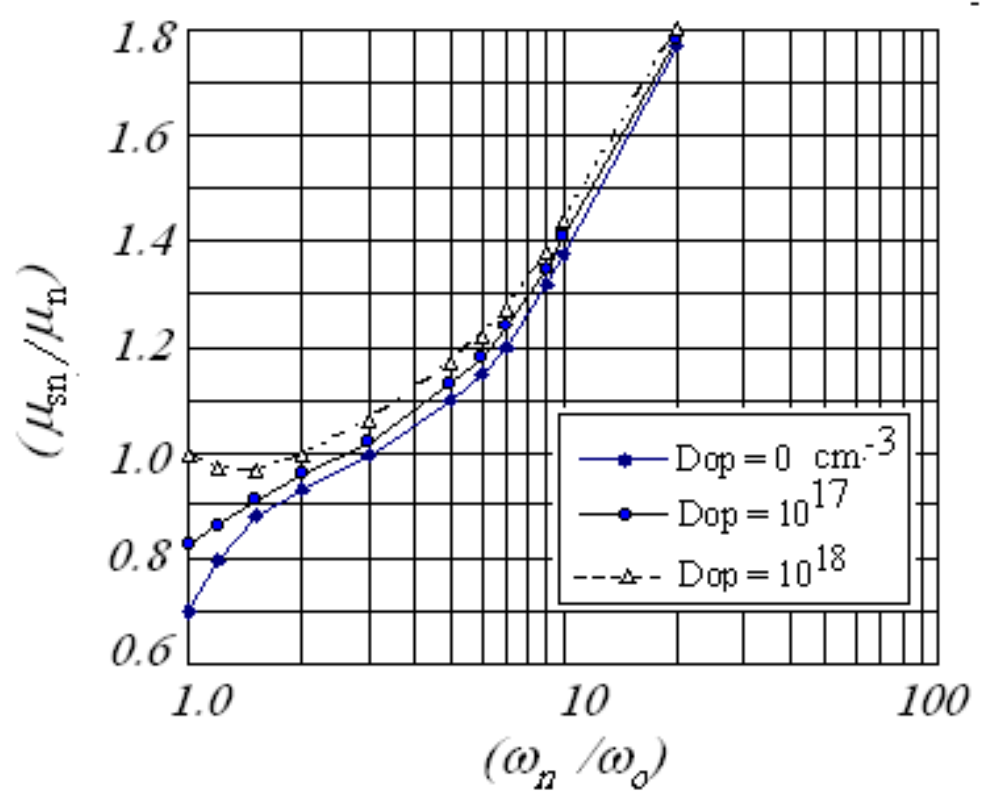

Figure 6(b). Relative energy flow mobility in $\mathrm{Si}$ at $300 \mathrm{~K}$, calculated from MC simulation, with non-parabolicty fact or $0.5 \mathrm{~V}-1$

\subsection{Advanced Heat Flux Model}

As we pointed out earlier, the heat flux term in the majority of previous HDM's was either neglected or modelled by the Fourier relation (12-a). It has been shown that this model is not accurate for modelling the heat flux across semiconductor interfaces in general and p-n junctions in particular[122]-[124]. In order to increase the accuracy of the hydrodynamic model, we propose the following model, which is based on the third moment of the BTE in steady state:

$$
n F .\left\langle\left(m_{n}^{*}\right)^{-1} E_{n}+u_{n} \otimes u_{n}\right\rangle+\nabla_{x} \bullet\left(n\left\langle E_{n} u_{n} \otimes u_{n}\right\rangle\right)=\left[\frac{\partial S_{n}}{\partial t}\right]_{c} \approx-\frac{S_{n}}{\tau_{s n}}
$$

where we suppressed the time derivative $\left(\partial S_{n} / \partial t\right)$ and used the relaxation time approximation $\left(\tau_{s n}\right.$ is the energy flux relaxation time). The above conservation equation (of energy flux), may be further simplified by approximating the average terms, as follows[123]:

$$
<\left(\mathrm{m}_{\mathrm{n}}^{*}\right)^{-1} \mathrm{E}_{\mathrm{n}}+\mathbf{u}_{\mathrm{n}} \otimes \mathbf{u}_{\mathrm{n}}>\approx 5 / 2 \alpha_{\mathrm{n}}<\mathbf{u}_{\mathrm{n}} \otimes \mathbf{u}_{\mathrm{n}}>,\left\langle\mathrm{E}_{\mathrm{n}} \mathbf{u}_{\mathrm{n}} \otimes \mathbf{u}_{\mathrm{n}}>\approx \beta_{\mathrm{n}} \mathrm{m}_{\mathrm{n}}\left(\left\langle\mathbf{u}_{\mathrm{n}} \otimes \mathbf{u}_{\mathrm{n}}>\right)^{2}\right.\right.
$$

where the correction factor $\alpha_{n}$, expresses the anisotropy of the distribution function and the correction factors $\beta_{n}$ expresses how much the distribution function is deviated from the Maxwellian distribution. These parameters can be estimated by MC simulation in the bulk of semiconductor. Then we can write the energy flux as the sum of a conduction part $\boldsymbol{Q}_{n}$ and a convection part (where $\boldsymbol{J}_{n}$ is a mu ltip lier):

$$
\mathbf{S}_{\mathrm{n}}=\mathbf{Q}_{\mathrm{n}}+5 / 2\left(\mu_{\mathrm{sn}} / \mu_{\mathrm{n}}\right)\left(\mathrm{k}_{\mathrm{B}} \mathrm{T}_{\mathrm{n}}\right) \cdot \mathbf{J}_{\mathrm{n}}
$$

Here $\mu_{s n}=\left(e m_{n}^{-1} \tau_{s n}\right)$ and the electron heat flux $\boldsymbol{Q}_{\mathrm{n}}\left(\underset{J_{n} \rightarrow 0}{\operatorname{Lim}} S_{n}\right)$ is given by:

$$
\mathbf{Q}_{\mathrm{n}}=-\mathrm{k}_{\mathrm{n}}{ }^{\prime} \nabla \mathrm{T}_{\mathrm{n}}-\mathrm{k}_{\mathrm{n}}{ }^{\prime}\left[\nabla\left(\mathrm{n} \mathrm{T}_{\mathrm{n}}\right) / \mathrm{n}\right]
$$

with

$$
\begin{gathered}
\mathrm{k}_{\mathrm{n}}{ }^{\prime}=5 / 2\left(\mathrm{k}_{\mathrm{B}}{ }^{2} / \mathrm{e}\right) \mathrm{n} \mu_{\mathrm{sn}} \beta_{\mathrm{n}} \mathrm{T}_{\mathrm{n}}, \\
\mathrm{k}_{\mathrm{n}}{ }^{2}=5 / 2\left(\mathrm{k}_{\mathrm{B}}{ }^{2} / \mathrm{e}\right) \mathrm{n} \mu_{\mathrm{sn}}\left(\beta_{\mathrm{n}}-\alpha_{\mathrm{n}}{ }^{\prime}\right) \mathrm{T}_{\mathrm{n}}
\end{gathered}
$$

Note that the convection energy flu $\mathrm{x}$ is similar to our 3-mo ments formu lation (11), except for the correction factor $\left(\mu_{s n} / \mu_{n}\right)$ and that $\left(\omega_{n}+k_{B} T_{n}\right)$ is replaced with $5 / 2\left(k_{B} T_{n}\right)$. However, we just called the third mo ment equation in order to derive a more accurate formu la of the heat flux term., which contains the effect of both the carrier temperature and the carrier density gradients.

\subsection{Modified Wiedman-Franz Relation}

If the anis otropy of the distribution function is negligible, we can substitute $\alpha_{n}, \approx 1$. Then the heat flux term can be put in the following simple form:

$$
\mathbf{Q}_{\mathrm{n}}=-5 / 2\left(\mathrm{k}_{\mathrm{B}}{ }^{2} / \mathrm{e}\right) \mu_{\mathrm{sn}}\left(2 \beta_{\mathrm{n}}-1\right) \nabla\left(\mathrm{nT}_{\mathrm{n}}\right)
$$

Figures 6(a) and (b) depict the electron heat flu $\mathrm{x}$ beta factor and the relative energy flux mobility $\left(\mu_{s n} / \mu_{n}\right)$ as a function of electron energy. We calculated these parameters by MC simulation in the bulk of Si at $300 \mathrm{~K}$ with non-parabolicity factor 
$0.5 \mathrm{~V}^{-1}$. Alternatively, they may be evaluated, as functions of carrier energy, and doping level, by appropriate physical models. For instance, the energy flu x mobility may be modelled in much the same way as the drift mobility as follows [124]:

$$
\mu_{s n}^{-1}=\mu_{\text {sno }}^{-1}\left[1+\xi_{n}\left(\omega_{n}-\omega_{o}\right)\right]
$$

where $\mu_{s n o}=\mu_{s n o}(D o p)$ is the energy flux mobility at low field and $\xi_{n}=5 / 2 n \mu_{s n o} \omega_{n} /\left(e \tau_{\omega n} v_{n} S_{n}\right)$. Also, the heat flux beta factor may be approximately expressed as follows in $\mathrm{Si}$ at $300 \mathrm{~K}$ :

$$
\beta_{n}\left(\omega_{n}, \text { Dop }\right)=\beta_{1}\left[1+\left(\operatorname{Dop} / N_{1}\right)^{\alpha_{2}}\right]^{-1}+\beta_{2}\left(\omega_{n}-\omega_{o}\right)\left[1+\left(\operatorname{Dop} / N_{1}\right)^{\alpha_{1}}\right]
$$

where $\alpha_{1}, \alpha_{2}, \beta_{1}, \beta_{2}$, and $N_{1}$ are constants. For $\mathrm{Si}$ at $300 \mathrm{~K}$ with doping levels Dop $\leq 10^{19} \mathrm{~cm}^{-3}$ and electron energy $\omega_{\mathrm{n}}>$ $0.1 \mathrm{eV}$, we have: $\beta_{1}=0.9775, \beta_{2}=6.464 \mathrm{eV}^{-1}, \alpha_{1}=0.192, \alpha_{1}=$ 6.372, $\mathrm{N}_{1}=10^{21} \mathrm{~cm}^{-3}$.

When the carrier-concentration gradient is negligible (with respect to the temperature gradient), the heat flux formula (35-a) can be further reduced to the conventional Fourier relation (12-a), with the following carrier-thermal conductivity:

$$
\mathrm{k}_{\mathrm{n}}=5 / 2\left(\mathrm{k}_{\mathrm{B}}{ }^{2} / \mathrm{e}\right) \mathrm{n} \mu_{\mathrm{sn}}\left(2 \beta_{\mathrm{n}}-1\right) \mathrm{T}_{\mathrm{n}}
$$

The above equation is equivalent to the conventional Wiedemann-Franz relation with the following 'modified' energy- and doping-dependent Lorenz nu mber:

$$
\gamma_{n}=5 / 2\left(\mu_{s n} / \mu_{n}\right)\left(2 \beta_{n}-1\right)
$$

In the extreme case of very low doping and very low electric field, where $\mu_{s n}=\mu_{n}$ and $\beta_{n}=1$ then $\gamma_{n}=5 / 2$ as compared to $(5 / 2+r)$ in the conventional model (12-c). Figure 13 depicts the heat flux as obtained by the classic Fourrier relation and the new model. As shown in figure, the consideration of the carrier gradients in the new model broaden and smooth the heat fluc profile near junctions.

\subsection{Tunneling \& Hot Carrier Injection Currents}

The tunnelling and hot carriers injection currents paly an important role in modern semiconductor devices in general and the ultra short MOSFET devices in particular. There are two tunnelling (through the barrier) mechanis $\mathrm{ms}$ and four hot carrier injection (over the barrier) mechanisms which are commonly encountered in MOSFET devices, namely:

- Field-assisted (Fowler-Nordheim or FN) tunnelling,

- direct tunnelling (DT),

- drain avalanche hot carrier injection (DAHCI),

- channel hot electron injection (CHEI),

- substrate hot electron injection (S HI) and

- secondary generated hot electron injection (S GHEI)

These mechanisms are usually monitored by measuring the gate current, $I_{G}$, and the substrate current, $I_{\text {sub }}$. In the following subsections we describe briefly these parameters and how they are modeled within the framework of the hydrodynamic model.

\subsubsection{Tunneling Currents}

The tunnelling currents are either direct or of field-assisted (Fowler-Nordheim) type. The direct tunnelling can take place at low gate voltages via thin oxides layers (less than $50 \AA$ ) and has a weak dependence on the gate field. The FN tunnelling is a field-assisted mechanism which is strongly dependent on the gate field and dominates in modern MOS structures. The gate tunnelling current density is given by the following integration[125]:

$$
J_{G}=\int_{0}^{\infty} g_{c}\left(E_{n}\right) \cdot u_{n \perp} f_{n}\left(E_{n}\right) \cdot T\left(E_{n}\right) \cdot d E_{n}
$$

where $T\left(E_{n}\right)$ is the tunneling probability, $g_{c}\left(E_{n}\right)$ is the density of states in the conduction band, $u_{n} \perp$ is the electron group velocity perpendicular to the Si surface $\left(u_{n} \perp \approx 1 / 4 u_{n}\right)$. The integration in the above equation starts from the semiconductor conduction band edge, as a reference. For trapezoidal barrier of upper height $\phi_{B}$ and lower hight $\left(\phi_{B}\right.$ $-V_{o x}$ ), where $V_{o x}$ is the potential voltage across the insulator gate, the tunnelling probability is usually expressed using the WKB approximation as follows:

$$
T\left(E_{n}\right)=\exp \left[-\frac{4 \sqrt{2 m_{o x}}}{3 e \hbar \zeta_{o x}}\left(e \varphi_{B}-E_{n}\right)^{3 / 2}\right]
$$

In the upper triangular part of the barrier, where e $\phi_{B}>E_{n}>$ $e\left(\phi_{B}-V_{o x}\right)$, and

$T\left(E_{n}\right)=\exp \left[-\frac{4 \sqrt{2 m_{o x}}}{3 e \hbar \zeta_{o x}} \cdot\left(\left(e \varphi_{B}-E_{n}\right)^{3 / 2}-\left(e \varphi_{B}-E_{n}-e V_{o x}\right)^{3 / 2}\right)\right]$

in the lower rectangular part of the barrier where $E_{n}<e\left(\phi_{B}\right.$ $\left.-V_{o x}\right)$. Here $\zeta_{o x}$ and $m_{o x}$ are the electric field and effective mass of electrons at the insulator (for $\mathrm{SiO}_{2}, m_{o x}=0.42 m_{0}$ ). In the case of the field-assisted Fowler-Nordheim mechanism, we consider the triangular part of the barrier, which is described by (38-a). Assuming the energy distribution function Maxwellian and the energy bands parabolic, we get the following field-dependent gate current density:

$$
J_{G}=J_{0} \cdot \zeta_{o x}{ }^{2} \exp \left[-\zeta_{0} / \zeta_{o x}\right]
$$

where the $J_{o}$ and $\zeta_{0}$ are constants, related to the energy barrier height $\phi_{B}$ between the insulator and the injecting conductor as follows:

$$
\begin{gathered}
\zeta_{o}=\frac{4 \sqrt{2 m_{o x}}}{3 e \hbar} \cdot\left(e \cdot \varphi_{B}\right)^{3 / 2} \\
J_{o}=\frac{e^{2}}{16 \pi^{2}\left(m_{o x} / m_{o}\right) \cdot \hbar \cdot \phi_{B}} .
\end{gathered}
$$

The tunnelling mechanis $m$ can be initiated by low energy carriers (near equilibrium). However, it is also possible for hot electrons to tunnel when their energy is not sufficient to surmount the barrier. In order to obtain more realistic results of the gate tunnelling current in short MOSFET devices, some authors assumed a non-Maxwellian distribution function and considered the a nonparaboicity of energy 
bands[126]. The total gate current may be partitioned between the source and drain, as follows:

$$
\mathrm{I}_{\mathrm{G}}=\mathrm{I}_{\mathrm{GS}}+\mathrm{I}_{\mathrm{GD}}
$$

where

$$
\begin{gathered}
I_{G S}=W \int_{0}^{L} J_{G}\left[1-\frac{y}{L}\right] d y, \\
I_{G D}=W \int_{0}^{L} J_{G}\left[\frac{y}{L}\right] d y
\end{gathered}
$$

where $W$ and $L$ are the MOSFET channel width and effective length, respectively. When the substrate bias is high enough, the thermoionic emission across the gate oxide dominates the tunnelling current. The thermoionic emission current can be calculated by a formula similar to equation (40), with replacing the the tunnelling probability, $T$, by the probability that an electron will not scatter in the image potential well in the oxide region, $P$.

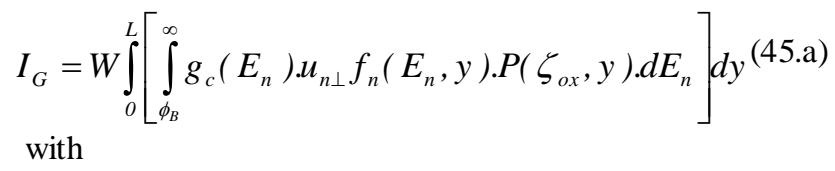

$$
\begin{gathered}
P=\exp \left(-\frac{x_{o x}}{\lambda_{o x}}\right) \text { for } \zeta_{o x}>0 \text { and } \\
P=\exp \left(-\frac{\left(t_{o x}-x_{o x}\right)}{\lambda_{o x}}\right) \text { for } \zeta_{o x}<0
\end{gathered}
$$

where $x_{o x}$ is the gate oxide thickness and $\lambda_{o x}$ is the mean free path of electrons inside the gate oxide (for $\mathrm{SiO}_{2}, \lambda_{o x} \approx$ $32 \AA$ ). In order to account for both tunnelling and thermo ionic emission, the above equation should be modified as follows [127]:

$$
I_{G}=W \int_{0}^{L}\left[\int_{0}^{\infty} g_{c}\left(E_{n}\right) \cdot u_{n \perp} f_{n}\left(E_{n}, y\right) \cdot T\left(E_{n}\right) \cdot P\left(\zeta_{o x}, y\right) \cdot d E_{n}\right] \cdot d y{ }^{(45 . c)}
$$

Finally it should be noted that the Fowler-Nordheim tunnelling gate current may photogenerate e-hole pairs at the substrate and hence it is likely to be the main origin of the substrate leakage current, rather than the anode hole injection[128].

\subsubsection{Hot Carrier Injection Currents}

The channel hot electron injection (CHEI) occurs when the gate voltage is high enough and $\mathrm{V}_{\mathrm{G}} \approx \mathrm{V}_{\mathrm{D}}$. Channel carriers which are accelerated by lateral fields and transported from the source to the drain may be driven towards the gate oxide before they reach the drain. The drain avalanche hot carrier injection (DAHCI) occurs when a high drain voltage is applied in non-saturation mode $\left(V_{D}>V_{G}\right)$ which results in a high electric field near the drain, and hence accelerating channel carriers into the drain depletion region. The accelerated channel carriers collide with $\mathrm{Si}$ atoms valence electrons, creating electron-hole pairs by impact ionization. Some of the generated electron-hole pairs are again accelerated and may acquire sufficient energy to surmount the $\mathrm{Si} / \mathrm{SiO}_{2}$ barrier. The hot carriers that surmount the gate oxide barrier can inject into the gate oxide where they may be trapped and gives rise to a shift in the MOSFET threshold voltage, surface mobility and dynamic conductance. The substrate hot electron injection (SHEI) occurs at high substrate bias, $\left|\mathrm{V}_{\mathrm{B}}\right| \gg>0$ and strong inversion, usually with both drain and source grounded. Under this condition, carriers of one type in the substrate are driven by the substrate field toward the $\mathrm{Si}-\mathrm{SiO}_{2}$ interface. Carriers can be generated by external optical or thermal excitation. As carriers move toward the substrate-oxide interface, they gain further kinetic energy fro $m$ the high field in surface depletion region. They eventually overcome the surface energy barrier and get injected into the gate oxide, where some of the m are trapped. The secondary generated hot electron injection (SGHEI) occurs under conditions similar to DAHCI, when $V_{D}>V_{G}$, which lead to impact ionization of hot carriers. However, SGHEI involves secondary carriers that are created by an earlier incident of impact ionization and driven under the influence of the substrate bias. This bias produces a field that drives hot carriers toward the surface region, where they gain more energy to overcome the gate oxide barrier[124]. The so-called "channel-initiated secondary electron (CHISEL) is a variant of the SGHEI, which relies on ionization feedback and is activated by a negative substrate bias $\mathrm{V}_{\mathrm{B}}<0$. CHISEL is used as a reliable programming technology in recent low-voltage Flash EEPROM devices, with floating gate lengths down to $0.2 \mu \mathrm{m}[131]$. Figure 18 illustrates the different hot injection and tunnelling mechanis ms in MOSFET devices.

The injection of hot-electrons into the gate-oxide has been usually modelled by the lucky-electron model[83]. According to $\mathrm{Hu}$ et al., the probability of a channel electron reaching the gate terminal as a combination of the probabilities of the following events:

- the electron gains sufficient energy in the lateral electric field to overco me the interfacial

- potential barrier and retains this energy after a collision directs its momentum towards the interface,

- the electron reaches the interface without suffering any more collisions, and

the electron is not scattered back into the semiconductor in the image-force potential well present close the interface

The lucky-electron model, despite of its drastic assumptions, remains the most useful approach to estimate the injection currents with reasonable agreement with experimental results. On the basis of the lucky-electron model, it can be shown that the fraction of the channel carrier supply which is injected into the gate oxide is given by [129] :

$$
I_{i n j} \propto I_{s u b} \exp \left(-\frac{\phi_{B}}{e \lambda_{n} \zeta}\right)
$$

where, $\phi_{B}$ is the interfacial potential barrier, $\lambda_{n}$ is the electron mean free path and $\zeta$ is the effective lateral electric field. It can be shown that the effective barrier potential $\phi_{B}$ is given by: 


$$
\varphi_{B}=\Phi_{B}-a \sqrt{\zeta_{o x}}-b \zeta_{o x}^{2 / 3}
$$

where $\Phi_{B}, a$ and $b$ are constants, expressing the main barrier height and lowering effects due to image force and quantum tunnelling. For $\mathrm{Si} / \mathrm{SiO}_{2}$ interface, $\Phi_{B}=3.2 \mathrm{~V}, a=$ and $b=2.59 \times 10^{-4}(\mathrm{Vcm})^{1 / 2}$. As the substrate current is generated due to impact ionization in the lateral electric field close to the drain, it is linearly proportional to the drain current. On the basis of the lucky-electron concept, it can be shown that the substrate currents is given by:

$$
I_{s u b} \propto I_{d s} \int_{0}^{L d} \alpha_{n} d y
$$

Where $I_{d s}$ is the drain current, $L_{d}$ is the length of the pinch-off region and $\alpha_{n}$ is the electron impact ionization coefficient in the velocity saturated part of the channel. So, the substrate current can be given by:

$$
I_{s u b}=C \cdot I_{d s} \exp \left(-\frac{E_{n}^{t h}}{e \lambda_{n} \zeta}\right)
$$

where, $E_{n}{ }^{\text {th }}$ is the impact ionization threshold energy for electrons. As seen from this equation, the ratio of the substrate and drain currents provides a direct measure of the average lateral electric field. In addition, this multiplication ratio, is often used as a monitor for the device degradation.

\section{Lattice Heat Conservation Equation}

Some recent studies demonstrated the important role of lattice heat and thermal activation of the low voltage impact ionization in deep-submicrometer MOSFETs[132]. In addition, the recent studies of silicon on insulator (SOI) devices by the classical HDM, showed certain anomalies due to lack of a heat evacuation mechanism at the insulator interface[133]. Some authors suggested to cure this problem by considering a tensorial temperature and modifying the closure condition for energy flux term [134]. Othors authors suggested coupling the lattice heat equation to the set of HD equations [135].

The classical heat diffusion equation, which resolves the temporal and spatial distribution of lattice temperature along the semiconductor, reads:

$$
\rho_{\mathrm{L}} c_{\mathrm{p}} \partial \mathrm{T}_{\mathrm{L}} / \partial \mathrm{t}-\nabla .\left(\mathrm{k}^{\mathrm{th}} \nabla \mathrm{T}_{\mathrm{L}}\right)=\mathbf{H}_{\mathrm{s}}
$$

where $\rho_{L}$ is the lattice density, $c_{p}$ is the lattice specific heat and their product is equal to the heat capacitance $\left(\mathrm{C}_{p}=\rho_{L} C_{p}\right)$ of the semiconductor material. Also $k^{\text {th }}$ is the thermal conductivity of the semiconductor and the heat source term $\boldsymbol{H}_{s}$ is given by[136]:

$$
\mathbf{H}_{\mathrm{s}}=\mathbf{J} . \zeta+\left[\left(\mathrm{E}_{\mathrm{c}}+\omega_{\mathrm{n}}\right)-\left(\mathrm{E}_{\mathrm{v}}-\omega_{\mathrm{p}}\right)\right](\mathrm{G}-\mathrm{R})
$$

where $\left(E_{c}+\omega_{n}\right)$ is the absolute average electron energy and $\left(E_{v}-\omega_{n}\right)$ is the absolute average hole energy in the crystal lattice. In $\mathrm{Si}$ at $300 \mathrm{~K}, \rho_{L}=0.232 \mathrm{~g} \mathrm{~m} / \mathrm{cm}^{3}$ and $c_{p}=7.03 \times 10^{4}$ $\mathrm{cm}^{2} / \mathrm{Ks}^{2}$ and their temperature dependence is negligible[137]. We dully note that the carrier thermal conductivity is a small fraction of the Si material thermal conductivity $k^{\text {th }}$ because heat conduction in semiconductors is dominated by phonon transport. It should be also noted that for $T_{L}>100 \mathrm{~K}$, the thermal conductivity in $\mathrm{Si}$ is practically independent of doping concentration[138]. The temperature dependence of $k^{\text {th }}$ in the bulk of crystalline Si may be modelled according to Glas sbenner and Slack[139] as follows:

$\mathrm{k}^{\text {th }}\left(\mathrm{T}_{\mathrm{L}}\right)=\mathrm{k}_{\mathrm{o}}\left(\mathrm{T}_{\mathrm{L}} / 300\right)^{-\alpha}(\mathrm{W} / \mathrm{K} \mathrm{cm})$ for $\mathrm{T}_{\mathrm{L}}>100 \mathrm{~K}$

where $\alpha=1.2$ and $k_{o} \approx 1.45$ for Si. However, in thin semiconductor layers the thermal conductivity is reduced due to the phonon scattering process at layer boundaries (the size effect), as described in [140]. An analytical expression describing the thermal conductivity as a function of distance, into thin Si layers can be found in[141]. A more rigorous approach to solve the problem of heat evacuation in SOI devices, consists in solving the BTE for phonons. In fact it is well known that the LO phonons have small group velocities $\left(\sim 10^{5} \mathrm{~cm} / \mathrm{s}\right)$ and anharmonically decay into faster acoustic phonons which in turn transport the energy out off the semiconductor device. So, the phonon BTE may be simplified by assuming a two-fluid phonon transport model involving a stationary optical reservoir mode and a propagating acoustic mode. The latter approach may consider not only the heat evacuation by optical phonons, but also their anharmonic decay to 'fast propagating' acoustic phonons. As a compromise between the above mentioned approaches, the a lattice heat continuity equation may be added to the set of HDE's with phonon energy relaxation term. The transformed phonon BTE in the relaxation time approximation reads [142]

$$
\frac{\partial \varpi}{\partial t}+\vartheta . \nabla \varpi=-\frac{\varpi-\varpi_{o}}{\tau_{\varpi}}+H_{s}
$$

where $\varpi$ is the phonon energy per unit volume, $v$ the phonon velocity and $\tau_{\varpi}$ is the phonon energy relaxation time and $H_{S}$ is the volumetric heat generation rate absorbed from hot electrons, which can be extracted from the solution of the HDE's. According to the kinetic theory, the phonon energy relaxation time is related to the phonon velocity $v_{p h}$ and mean-free path $\Lambda_{p h}$, by the following relation[143]:

$$
A_{p h}=v_{p h} \tau_{\varpi}=\frac{3 k_{B}}{C_{p} v_{p h}}
$$

So, the lattice energy conservation equation may be written as follows:

$$
\frac{\partial W_{L}}{\partial t}+\nabla \cdot S_{L}=H_{s}-\frac{W_{L}-W_{o}}{\tau_{\varpi}}=R_{\omega L}
$$

where $W_{L}=\int C_{v} d T_{L}$ is the lattice energy density, $W_{o}$ is the lattice energy density at equilibriu m (at $T_{L}=T_{o}$ ) and $S_{L}=-k^{\text {th }} \nabla$ $T_{L}$ is the lattice energy flux (or heat flux). We note that the overall lattice energy conservation dictates the following relation:

$$
\begin{aligned}
\mathrm{R}_{\omega \mathrm{L}}= & \mathrm{R}_{\omega \mathrm{n}}+\mathrm{R}_{\omega \mathrm{p}}=\zeta . J+\left(\mathrm{E}_{\mathrm{g}}+\omega_{\mathrm{n}}+\omega_{\mathrm{p}}\right)(\mathrm{G}-\mathrm{R}) \\
& -\mathrm{n}\left(\omega_{\mathrm{n}}-\omega_{\mathrm{o}}\right) / \tau_{\mathrm{wn}}-\mathrm{p}\left(\omega_{\mathrm{p}}-\omega_{\mathrm{o}}\right) / \tau_{\mathrm{wp}}
\end{aligned}
$$

where $R_{\omega n}$ is equal to the RHS of the energy conservation equation (10-c) for electrons and $R_{\omega p}$ for holes. When the net 
generation-recombination rate is negligible, $R_{\omega L}$ represents the local energy transfer rate from crystal lattice to the electron and hole gaz subsystems. Therefore we can write the following correlation function between the charge carriers relaxation times and phonons relaxation time:

$$
\mathrm{n}\left(\omega_{\mathrm{n}}-\omega_{\mathrm{o}}\right) / \tau_{\mathrm{wn}}+\mathrm{p}\left(\omega_{\mathrm{p}}-\omega_{\mathrm{o}}\right) / \tau_{\mathrm{wp}}=\left(\mathrm{W}_{\mathrm{L}}-\mathrm{W}_{\mathrm{o}}\right) / \tau_{\text {б }}
$$

Note that our formulation of the lattice heat conservation equation is tightly coupled with the set of HDEs, via the average electron and hole average energies (which makes part of the heat source term $\mathrm{H}_{\mathrm{s}}$ ) and the energy relaxation correlation function.

\section{Quantum Corrections}

The so-called quantum hydrodynamic model (QHDM) can be derived by a variety of methods [144-152], such as the first few moments of the WBTE or using the Madelung transform[147] or the principle of minimum entropy [148]. The formulation of the QHDM usually retains the lowest order $\left(\hbar^{2}\right)$ term in the series expansion of a complicated quantum operator acting on the Wigner distribution function and the system potential energy. From the mathematical point of view, the most significant difference between the semic lassical HDM and the QHDM is the quantum potential term, $\mathrm{V}_{\mathrm{q}}$, which appears in the average energy expression[150]:

$$
\omega_{\mathrm{n}}=1 / 2 \mathrm{~m}_{\mathrm{n}}^{-1}\left\langle\mathrm{p}_{\mathrm{n}}\right\rangle^{2}+3 / 2 \mathrm{k}_{\mathrm{B}} \mathrm{T}_{\mathrm{n}}+\mathrm{V}_{\mathrm{q}}
$$

where $\left\langle p_{n}\right\rangle$ is the average mo mentum of electrons and $V_{q}$ is given by:

$$
\mathrm{V}_{\mathrm{q}}=-\hbar^{2} \nabla^{2}[\ln \mathrm{n}] / 8 \mathrm{~m}_{\mathrm{n}}
$$

The quantum potential term is sometimes merged with the electron temperature, to form the so-called quantum temperature $T_{q n}$ :

$$
\mathrm{T}_{\mathrm{qn}}=\mathrm{T}_{\mathrm{n}}+2 / 3 \quad \mathrm{~V}_{\mathrm{q}} / \mathrm{k}_{\mathrm{B}}
$$

The quantum correction appears also in the current equation (which is a reduced form of the momentum conservation equation) in the form of an additional current $J_{q}$. The following electron current equation depicts this correction term[151]:

$$
\text { with }
$$

$$
\mathbf{J}_{\mathrm{n}}(\text { quantum })=\mathbf{J}_{\mathrm{n}}(\text { semic las sical })+\mathbf{J}_{\mathrm{q}}
$$

$$
J_{q}=n \mu_{n}\left(\frac{\hbar^{2}}{6 m_{n}^{*}}\right) \cdot \nabla\left(\frac{\nabla^{2} \sqrt{n}}{\sqrt{n}}\right)
$$

Note that the quantum correction term is significant when the electron concentration $n$ varies so rapidly over small distances in the order of de-Broglie wavelength $(h / p)$. Adding the quantum correction term in this manner is sometimes referred to as the density gradient (DG) method. The DG theory has been used to model the quantum confinement as well as the tunnelling currents in MOS devices [152].

\section{Quasi-Ballistic Transport Considerations}

It has been shown that the operation of sub 0.1 micron MOSFET transistors is quasi-ballistic, in a small portion of the channel[153-158]. Some authors shows that quasi-ballistic transport in nano-transistors takes place near the source region and others showed that quasi-ballistic transport and back scattering take also place near drain region as well[157]. In all cases, one should be aware that even when the transport is full ballistic, the contact resistance of the device is still there limiting the transport process. However, the quasi-ballis tic transport can be also considered within the framework of the semiclassical theory[156]. In fact, it has been shown that semiclassical transport models along the MOSFET channel may remain reliable down to $10 \mathrm{n} \mathrm{m}[157]$. In addition, it has been shown in the latter article, that the semiclassical model is more reliable than the quantum simulation, even when the ratio of coherent transport along the MOSFET channel is not negligeable (down to $10 \mathrm{~nm}$ ). Figure 7 depicts the electron velocity distribution along a $50 \mathrm{~nm} n-\mathrm{i}-\mathrm{n}$ structure, in the ballistic mode and using different transport approaches.

\section{Sample of Simulation Results}

Figure 1 depicts the electron temperature $T_{n}$, according to Monte Carlo simulation, the classic HDM and according to our defin ition. As shown in figure, the electronic te mperature is reasonably estimated using our model when taking $g_{n} \approx 1$, for electric field up to $200 \mathrm{kV} / \mathrm{cm}$. For the matter of demonstration of the proposed HDM and comparison with other DDM-based simulation, we simulated the carrier transport across a reverse-biased silicon $\mathrm{p}-\mathrm{i}-\mathrm{n}$ diode. The simulated diode is $100 \mu \mathrm{m}$ long and has a $\mathrm{p}^{+}$side with Gaussian distribution and peak doping of $10^{19} \mathrm{~cm}^{-3}$., as shown in Figure 8. The diode base $\mathrm{n}^{-}$-layer is homogenously doped with $10^{14} \mathrm{~cm}^{-3}$, and the metallurgical junction is almost at $11 \mu \mathrm{m}$ apart from the $\mathrm{p}^{+}$contact. Figures $\mathbf{9}$ and $\mathbf{1 0}$ depict the two-dimensional distributions of electron and hole density and temperatures inside the $p$-i-n diode at $75 \mathrm{~V}$ reverse bias. Figures 11 and $\mathbf{1 2}$ depict the one-dimensional distributions of electron and hole density and temperature along the $\mathrm{p}$-i-n diode at $500 \mathrm{~V}$ reverse bias. Also figures $\mathbf{1 3}$ and 14 depict the one-dimensional distributions of electron and hole energy flux and heat flux along the p-i-n diode at $500 \mathrm{~V}$ reverse bias. In addition. Figure 15 shows the I-V characteristics of the simulated diode near breakdown. We discussed in [159] the effect of the hydrodynamic simulation on the distribution of charge carriers and showed that discrepancy from the DDM-based results is due to the carrier thermalback diffusion. In order to show the important role of the energy relaxation time model in the HD simulation, we traced the electron distribution across the $\mathrm{p}^{+}-\mathrm{n}$ junction at various values of the $\tau_{w n}$. Figure 16 depicts the electron density distribution along a reverse biased $\mathrm{p}$-i-n diode at three values of the energy relaxation time. Note that the electron distribution, which is obtained using the HDM with lower $\tau_{\omega n}$ tends to the DDM classical distribution. This 
means that for shorter energy relaxation times, the electrons are more efficient in dissipating their energy. For the limiting case when $\tau_{\omega n}$ tends to zero, the electrons will tend to dissipate their gained energy at once and their average energy is equal the lattice energy $\omega_{o}$. This is exactly the case of the DD simulation, where we consider the electron temperature equal to the lattice temperature $\left(T_{n}=T_{L}\right)$ at all fields.
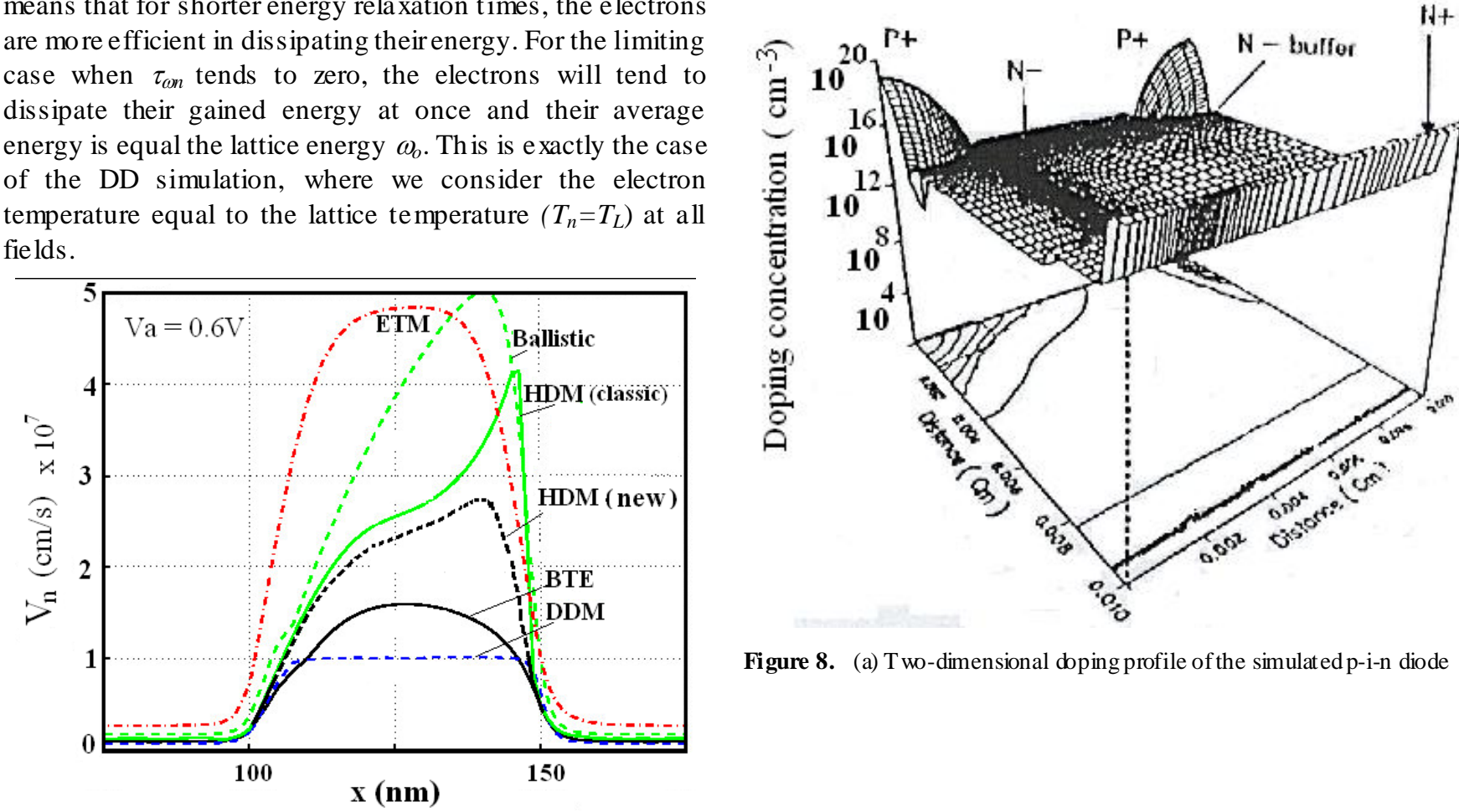

Figure 8. (a) Two-dimensional doping profile of the simulated p-i-n diode

Figure 7. Effect of quasi-ballistictransport on the electron velocity across an $n$-i-n diode, according to several models[118]

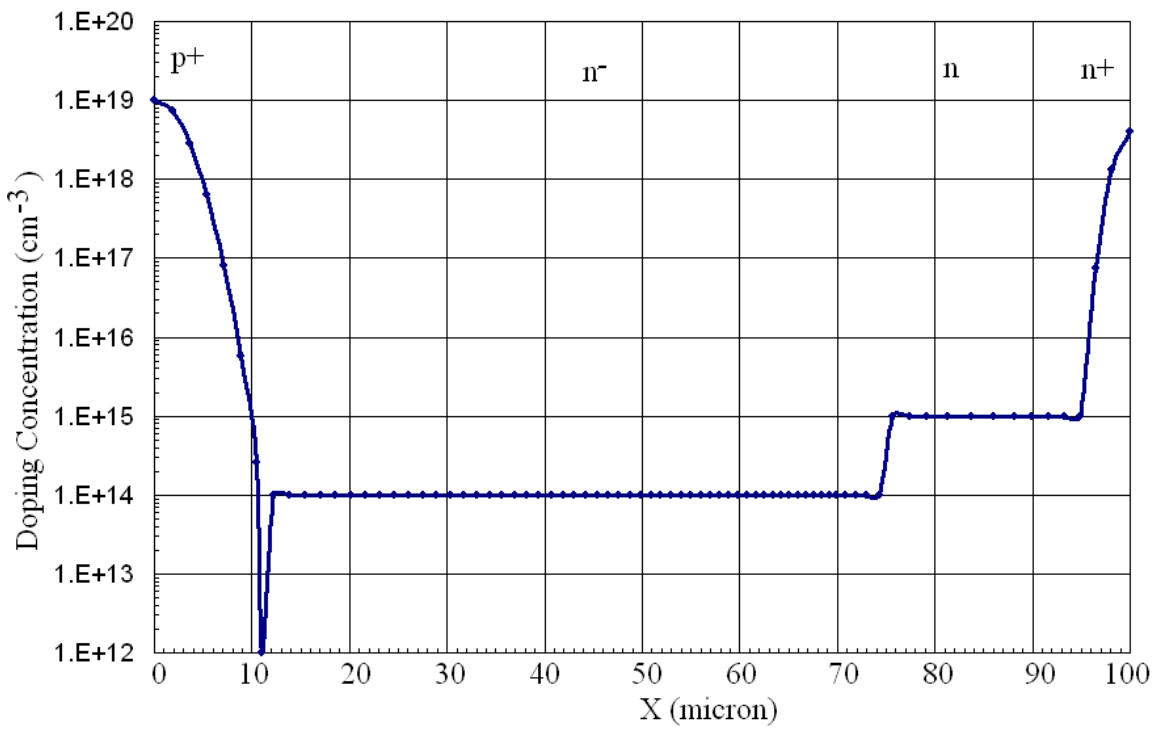

Figure 8. (b) Lateral doping profile along the $x$-axis of the $p-i-n$ diode
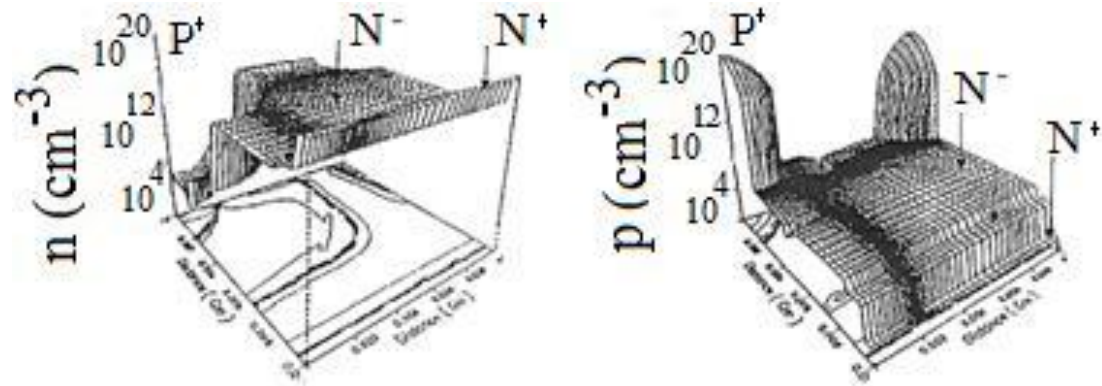

Figure 9. (a) Electron and (b) hole density distribution in the p-i-n diode (at $-75 \mathrm{~V}$ bias) 

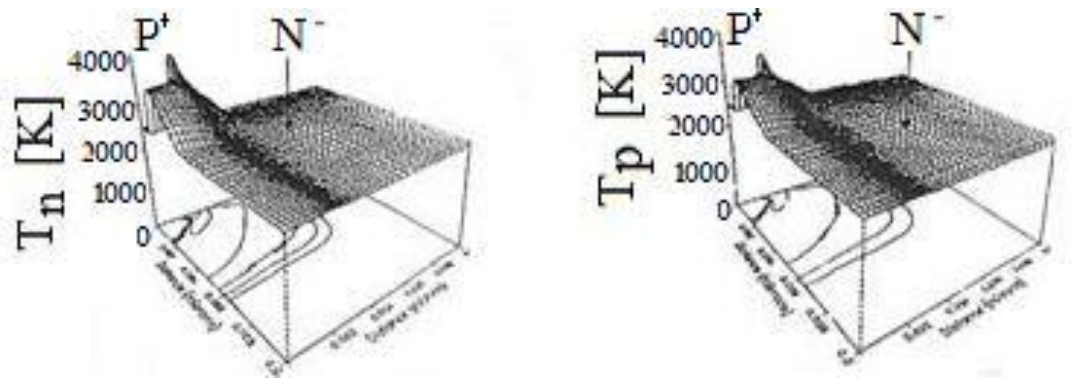

Figure 10. (a) Electron and (b) hole temperat ure distribution in the p-i-n diode (at $-75 \mathrm{~V}$ bias, $300 \mathrm{~K}$ )

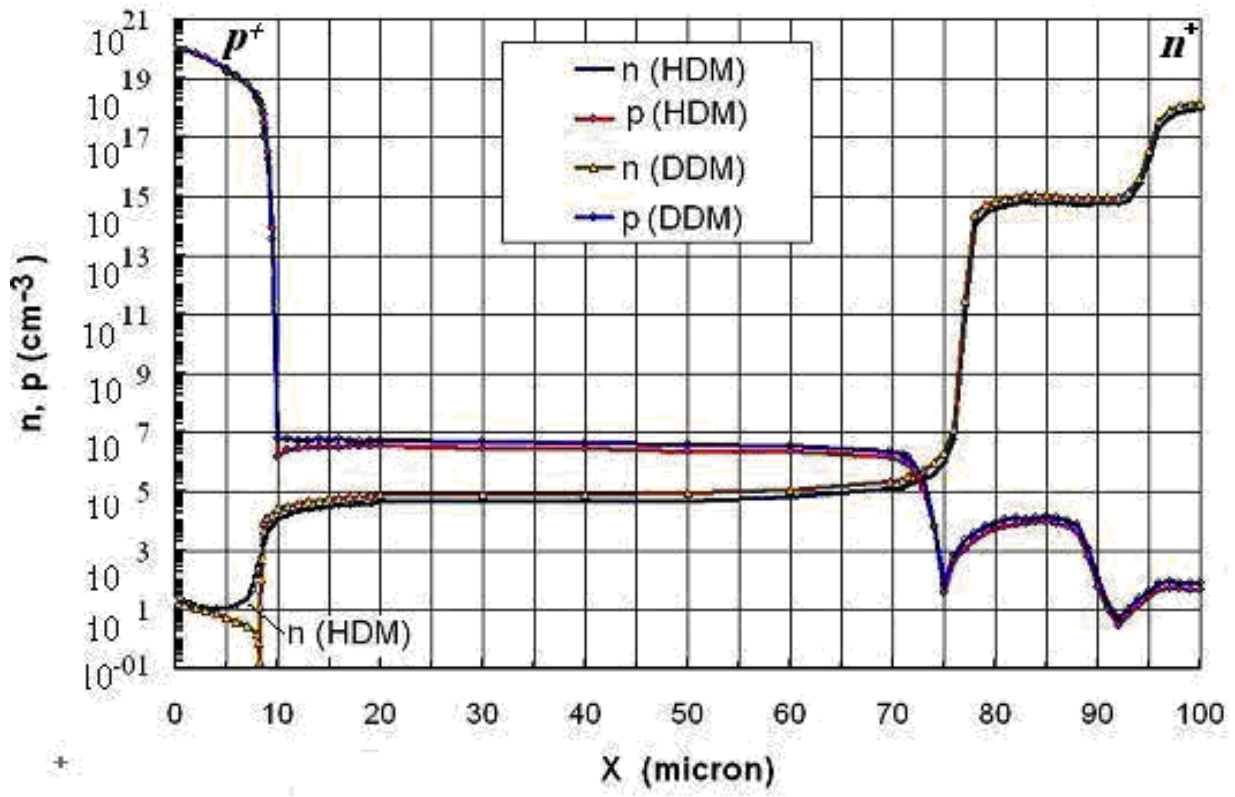

Figure 11. Electron density distribution along the p-i-n diode (at 500V reverse bias, 300K), on the basis of DDM and HDM

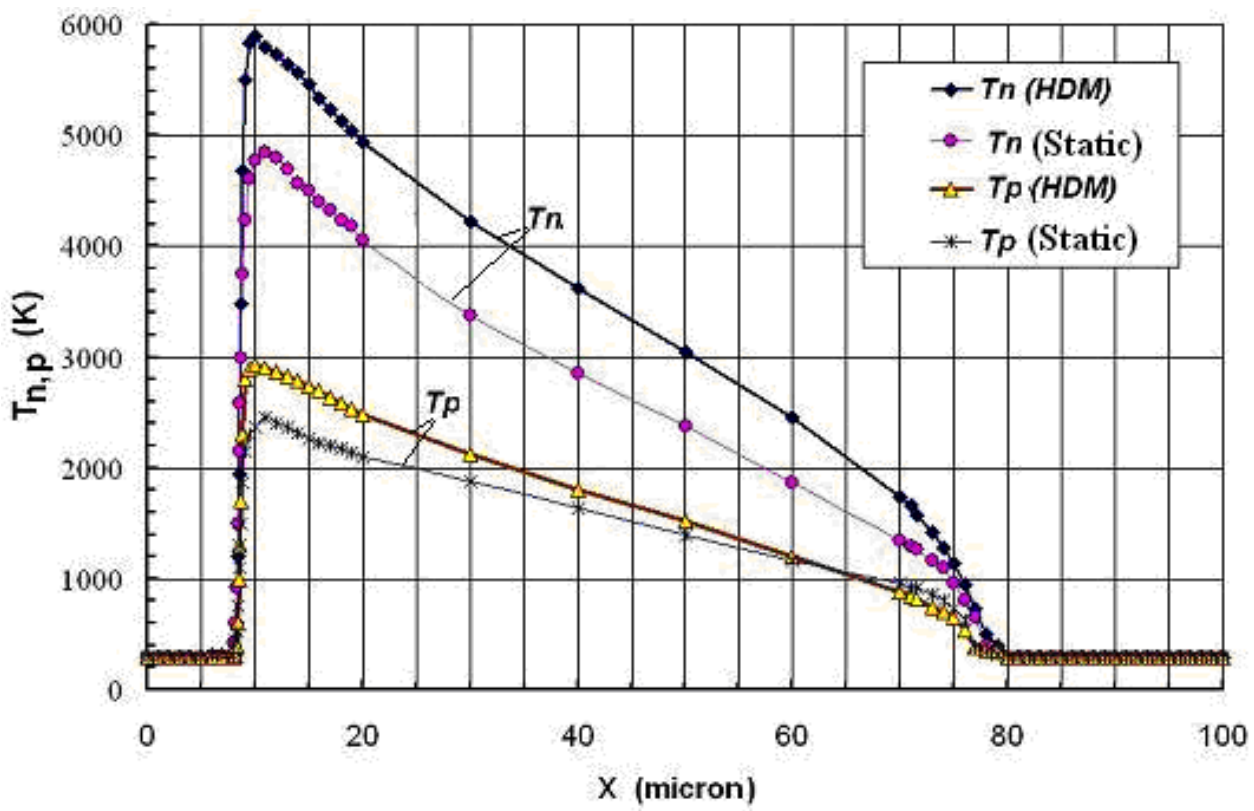

Figure 12. Electron and hole temperat ure distribution along a reverse biased $\mathrm{p}-\mathrm{i}-\mathrm{n}$ diode (at $-500 \mathrm{~V}$ ), as calculated from the DDM electric field distribution (static) and by HD simulation 


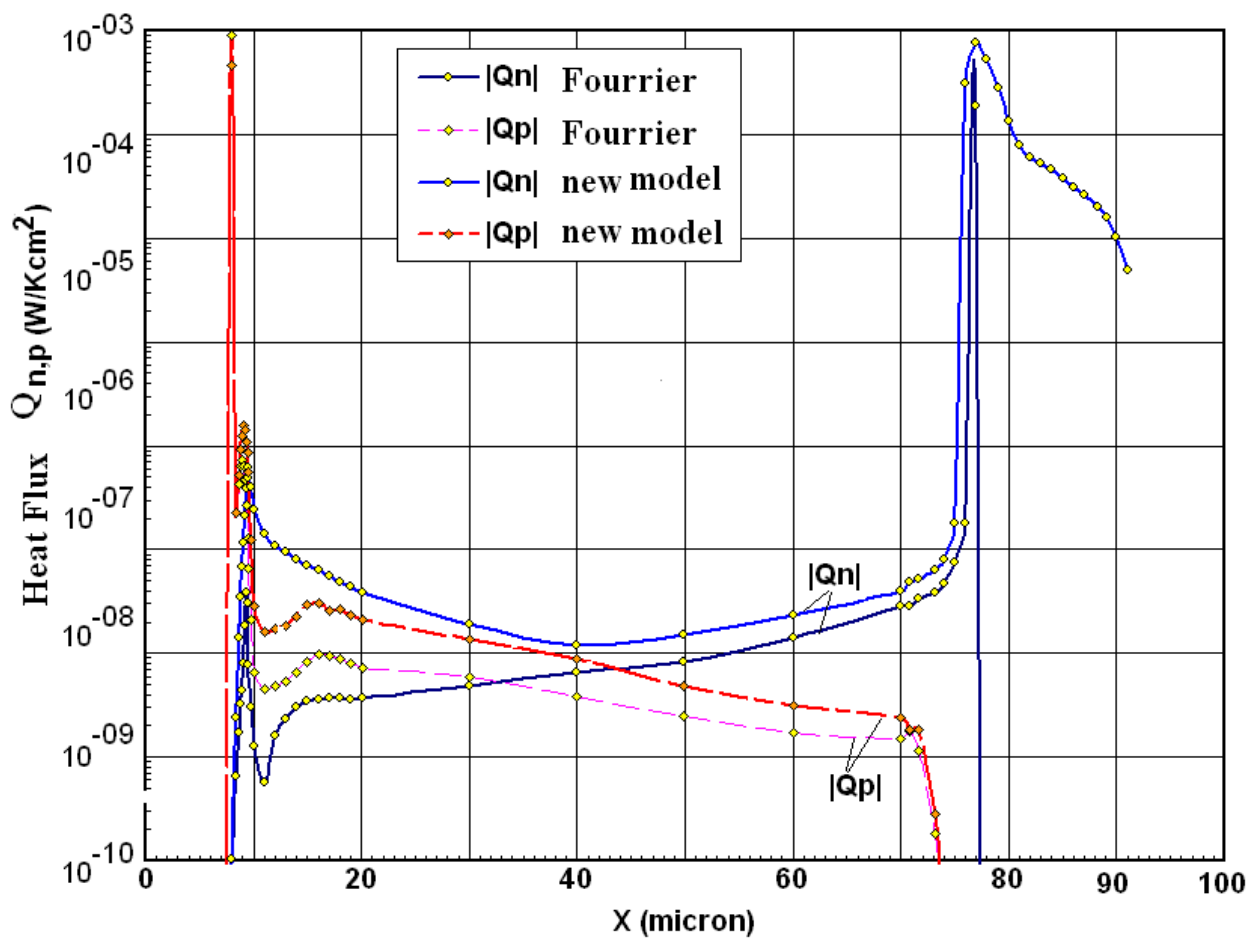

Figure 13. Electron heat flux distribution along the $\mathrm{p}-\mathrm{i}-\mathrm{n}$ diode at $500 \mathrm{~V}$ reverse bias, $300 \mathrm{~K}$

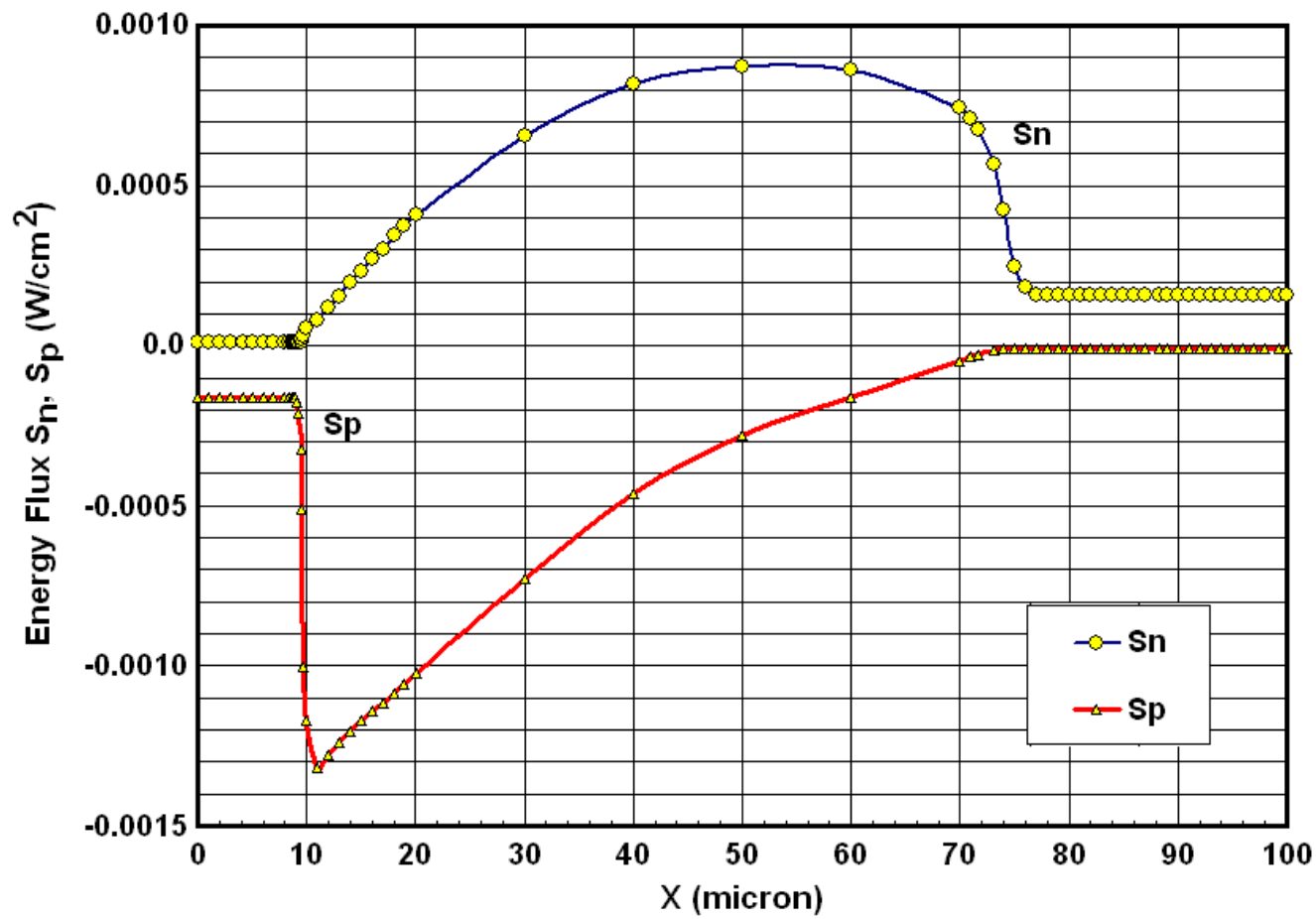

Figure 14. Electron energy flux distribution along the p-i-n diode at $500 \mathrm{~V}$ reverse bias, $300 \mathrm{~K}$ 


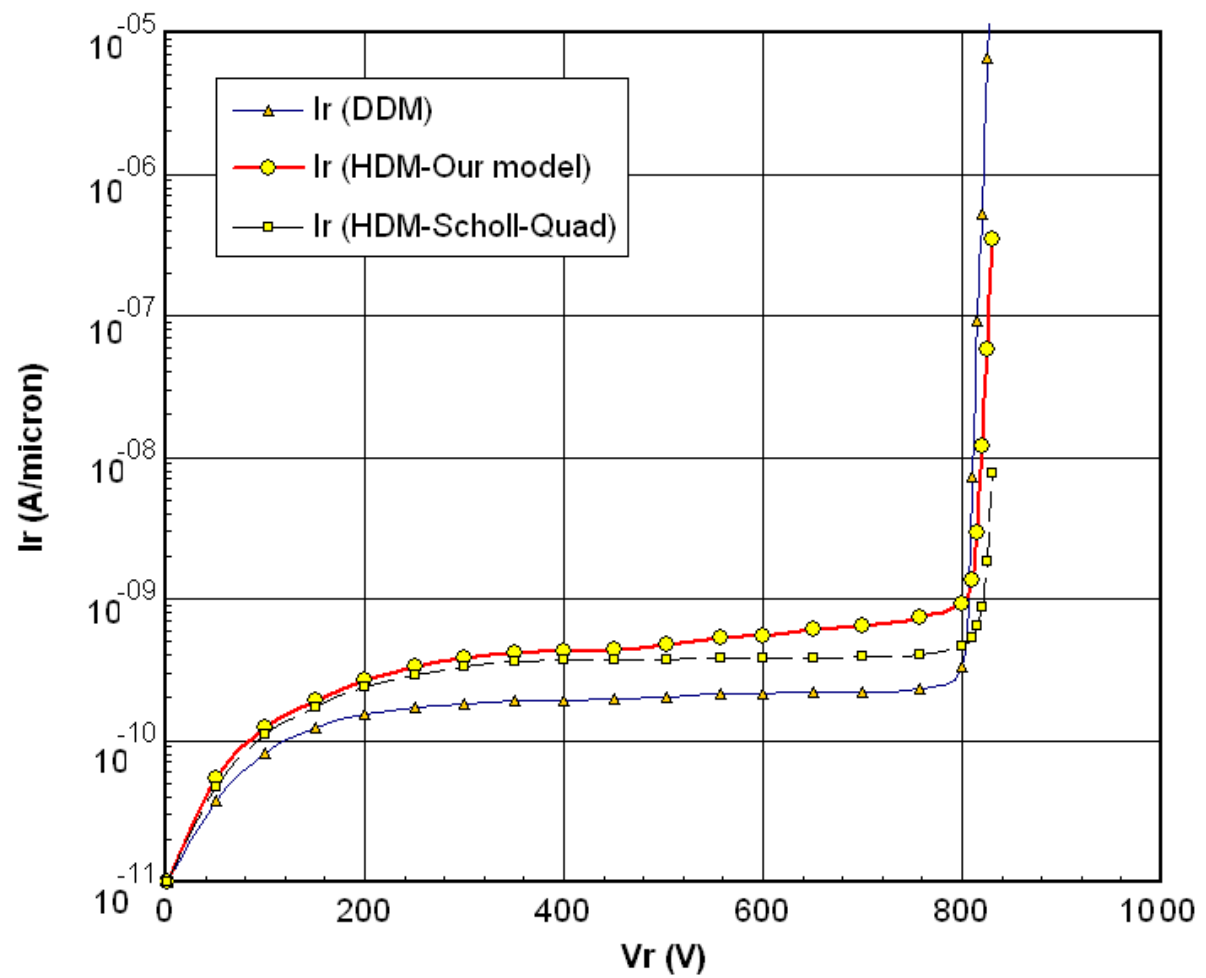

Figure 15. The I-V characteristics of the simulated the p-i-n diode near avalanche breakdown, as obtained by 2-dimmensional DD and HD simulation at $300 \mathrm{~K}$. The surface of the p-i-n diode is properly passivated and protected by field plates, so that the breakdown occurs at the junction curvat ure inside the p-i-n diode

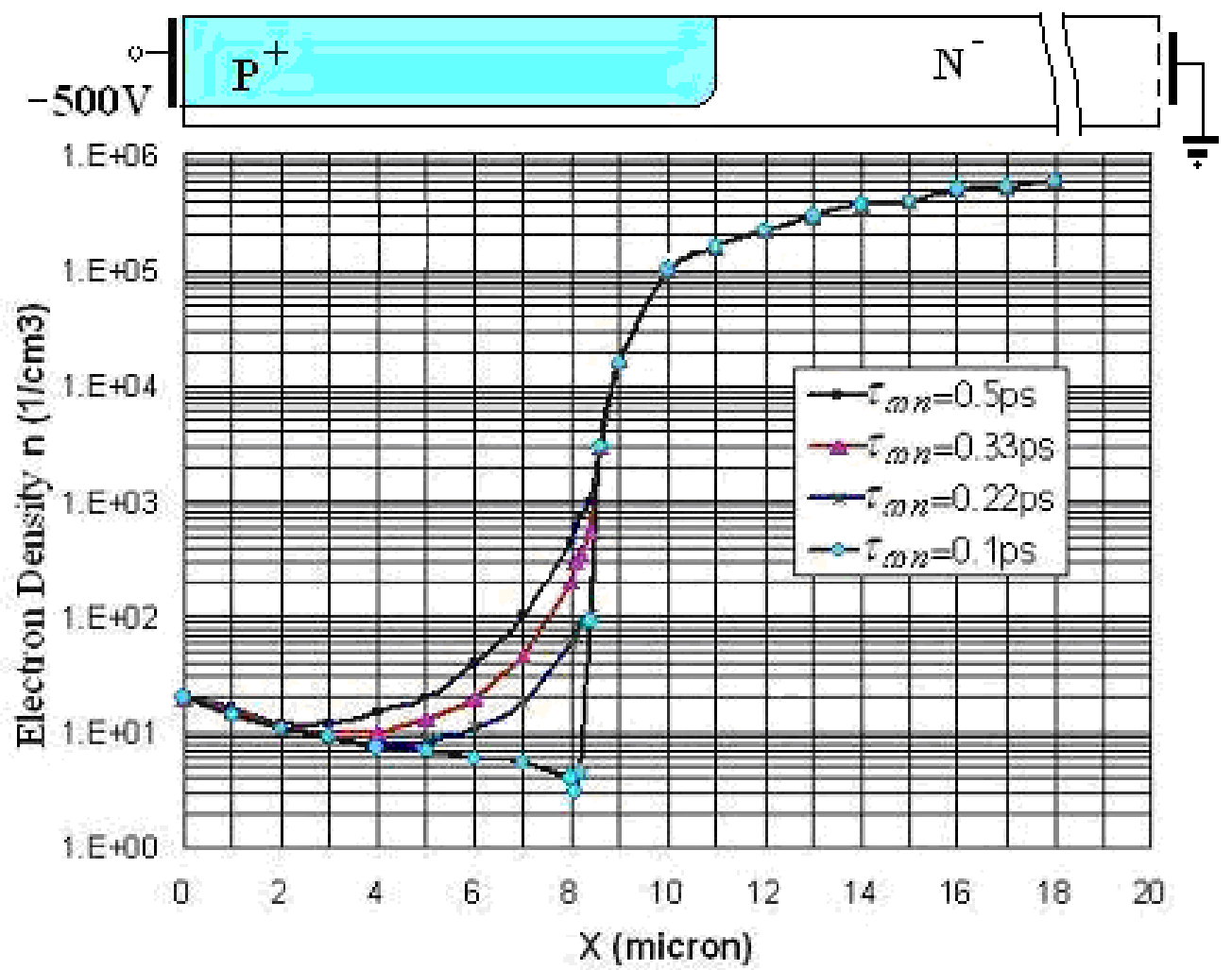

Figure 16. Electron density distribution along a reverse biased p-n junction, with different values of energy relax at ion time 


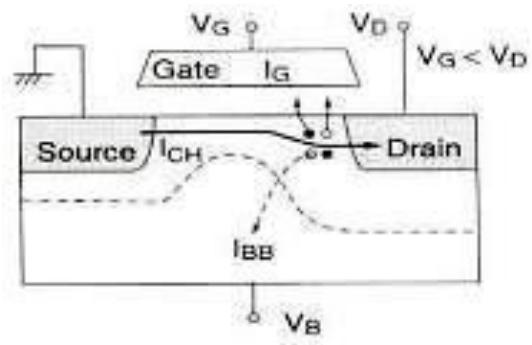

$\mathrm{CHI}(\mathrm{VG} \approx \mathrm{VD})$

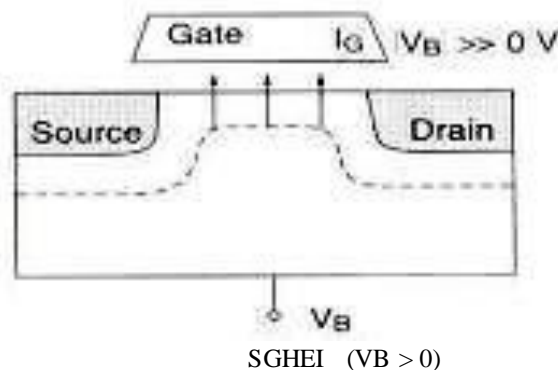

Figure 17. Illustartion of different hot carrier injection mechanisms in MOSFET Devices

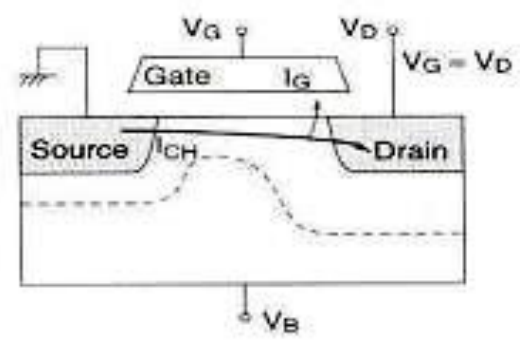

DCHEI $\quad(\mathrm{VG} \approx 1 / 2 \mathrm{VD})$

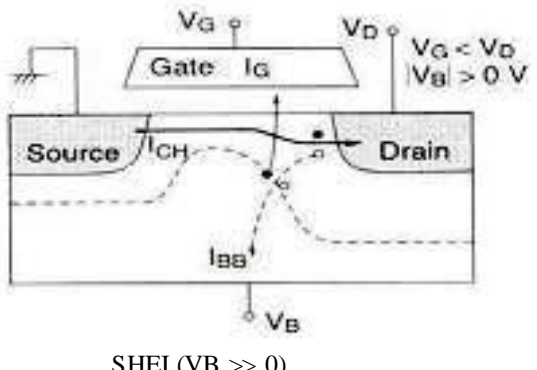

$\omega_{\mathrm{n}}=\omega_{\mathrm{no}}+\mathrm{e} \cdot \tau_{\mathrm{wn}} \mathrm{v}_{\mathrm{d}} \zeta$
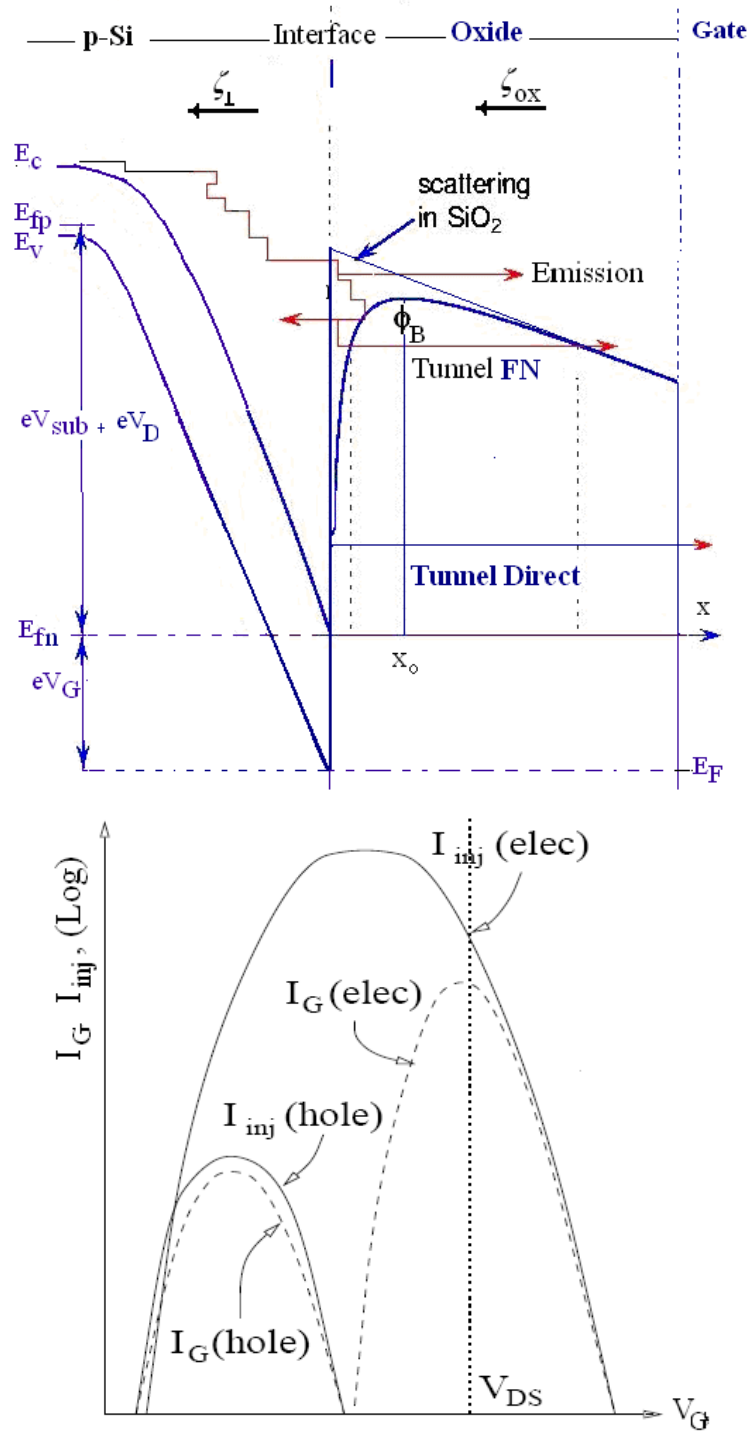

Figure 18. Illustration of hot carrier injection currents in MOSFET Devices
We may conclude from this discussion that the DDM simulation is a hypothetical case of the HDM where we assume $\tau_{\omega n}=0$ and hence $\omega_{n}=\omega_{o}$, and this case means a virtual semiconductor whose collisions are so efficient that electrons relax to their equilib riu m energy in almost no time.

$$
D D M=\operatorname{Lim}_{\tau a n \rightarrow 0} H D M
$$

The electrons in such a virtual semiconductor (with zero energy relaxation time) will not allowed to heat up even at high electric fields such that the mean electron energy $\omega_{n}$ will be almost equal to the lattice energy $\omega_{n o}$. This result has been already reported for majority carriers in MOSFET devices[97], but has never been reported for minority carriers in bipolar devices, as far as the author knowledge.

\section{Conclusions}

In conjunction with sophisticated Monte Carlo methods that incorporate both realistic band structure and carrier scattering processes, the HDM is a basic tool to understand hot carrier behaviour in ever smaller and ultra fast semiconductor devices. We presented in this article a complete hydrodynamic model based on the first three moments of the semiclassical BTE for electrons, holes and phonons, with a few number of parameters. The model includes the band structure non-parabolicity effects and advanced energy-dependent models of the carrier mobility, impact ionization rate and heat flux. As the accurate estimation of the carrier temperature in semiconductor devices, influences the estimation of so many important parameters, such as the breakdown voltage and leakage currents, we proposed a simple definition of the carrier temperature, which takes the band structure into account. The definition results in temperatures which are closer to the Monte Carlo simulation results than those obtained by the 
classical hydrodynamic models. We also proposed a phenomenological correlation between the electron average mass and energy relaxation time which cuts down the number of parameters of the HDM. In conclusion, our proposed system of HDE's has a few energy-dependent parameters $\left(\mu_{n}, \alpha_{n}, m_{n}, \tau_{\omega n}\right)$ and they are all correlated to the energy relaxation time $\tau_{\omega n}$. The later parameter can be obtained from the MC simulation[161] or by measurement. The proposed model has been applied to investigate the carrier trans port in certain semiconductor devices and led to adequate results. From our simulation, we conclude that the undeliberated choice of the energy relaxation time value in the HD simulation leads to drastic results and may be to the failure of the HDM in certain cases, as reported in [47]. For instance, we show, by simulation, that reducing the energy relaxation time to unreasonable short values will mask the thermal diffusion mechanis $m$ and gives erroneous results, in both MOSFET and bipolar devices .

\section{APPENDIX: Moments of the BTE}

Consider the distribution function $f(x, k, t)$, which obeys the BTE. Consider $A(k)$ a microscopic quantity, which is a function of the wave vector $\boldsymbol{k}$. Now, let's multiply the BTE by $A(k)$ and integrate the two sides of the equation over the entire k-space:

$$
\int A \frac{\partial f}{\partial t} d^{3} k+\int A v_{g} \bullet \nabla_{x} f . d^{3} k+\int \frac{A F}{\hbar} \bullet \nabla_{k} f d^{3} k=\int A\left(\frac{\partial f}{\partial t}\right)_{c} d^{3} k
$$

We have also, the mean value of $A(k)$, which we note by $\langle A\rangle$ is given by:

$$
\langle A\rangle=\frac{\int A(k) f(k, r, t) d^{3} k}{\int f(k, r, t) d^{3} k}=\frac{\int A(k) f(k, r, t) d^{3} k}{n}
$$

Therefore, the $1^{\text {st }}$ term of the L.H.S of equation (55) becomes:

$$
\int A \frac{\partial f}{\partial t} d^{3} k=\frac{\partial}{\partial t} \int A f d^{3} k=\frac{\partial(n\langle A\rangle)}{\partial t}
$$

The $2^{\text {nd }}$ term of the L.H.S of equation (55) becomes:

$$
\int A v_{g} \bullet \nabla_{x} f d^{3} k=\nabla_{x} \bullet \int A v_{g} f d^{3} k-\int \nabla_{x} A \bullet v_{g} f d^{3} k=\nabla_{x} \bullet\left(n\left\langle A v_{g}\right\rangle\right)
$$

Table 1. The integration of different terms of the LHS of the BTE, after being multiplied by A(k)

\begin{tabular}{|c|c|c|c|c|}
\hline Moment & $A(k)$ & $n\langle A\rangle$ & $\left\langle\left(F \bullet \nabla_{k}\right) A / \hbar\right\rangle$ & $\nabla_{x} \bullet\left(n\left\langle v_{g} A\right\rangle\right)$ \\
\hline 0 & 1 & $n$ & 0 & $\nabla_{x} \bullet\left(n v_{n}\right)$ \\
\hline $\boldsymbol{I}$ & $\boldsymbol{u}_{g}(k)$ & $n\left\langle v_{g}\right\rangle=n v_{n}$ & $-\frac{1}{\hbar^{2}}\left\langle\nabla_{k}^{2} E\right\rangle F=-\left\langle m^{*-1}\right\rangle F$ & $\nabla_{x} \bullet\left(n\left\langle v_{g} \otimes v_{g}\right\rangle\right)$ \\
\hline 2 & $E_{n}(k)$ & $n\left\langle E_{n}\right\rangle=n \omega_{n}$ & $-n v \cdot \boldsymbol{F}$ & $\nabla_{x} \bullet\left(n\left\langle E_{n} v_{g}\right\rangle\right)=\nabla \bullet S_{n}$ \\
\hline 3 & $\boldsymbol{u}_{g}(k) E_{n}(k)$ & $n\left\langle u_{n} E_{n}\right\rangle=S_{n}$ & $-\left\langle m^{*-1} E_{n}+u_{n} \otimes u_{n}\right\rangle F$ & $\nabla_{x} \bullet\left(n\left\langle E_{n} v_{g} \otimes v_{g}\right\rangle\right)$ \\
\hline
\end{tabular}

Also, the $3^{\text {rd }}$ term of the L.H.S of equation (55) becomes:

$$
\int \frac{A F}{\hbar} \bullet \nabla_{k} f d^{3} k=\sum_{j}\left|\frac{f(k, x, t) A F_{j}}{\hbar}\right|_{-\infty}^{\infty}-\int f \nabla_{k} \bullet\left(\frac{A F}{\hbar}\right) d^{3} k
$$

where we effectuated an integration by parts on the k-variable. The index $j$ of the force component $F_{j}$ appears for any orthonormal basis (e.g. for Cartesian coordinates, $j=x, y, z$ ). The totally integrated term in equation (I-2c) d is appears because $\operatorname{Lim}_{k \rightarrow \pm \infty} f(k, x, t) A(k) F_{j}=0$. This means that all the moments of the BTEare finite. On the otherhand, the second term of (I-2c) may be written as follows: 


$$
\left\langle\nabla_{k} \bullet\left(\frac{A F}{\hbar}\right)\right\rangle=\left\langle\left(\frac{F}{\hbar} \bullet \nabla_{k}\right) A\right\rangle+\left\langle\Delta \nabla_{k} \bullet\left(\frac{F}{\hbar}\right)\right\rangle=\left\langle\left(\frac{F}{\hbar} \bullet \nabla_{k}\right) A\right\rangle
$$

Here, we put $\nabla_{k} \bullet F=0$, which is true if $\boldsymbol{F}$ is independent of $k$ or when $\boldsymbol{F}$ is normal to $\boldsymbol{k}$. Fortunately, this is the case for electric field force ( $F=-e \zeta$ ) and Lorentz's force ( $F=-e v_{g} x B$ ). Now, by regrouping all (55) components we get:

$$
\frac{\partial}{\partial t} n\langle A\rangle+n\left\langle\left(\frac{F}{\hbar} \bullet \nabla_{k}\right) A\right\rangle+\nabla_{x} \bullet\left(n\left\langle v_{g} A\right\rangle\right)=\left[\frac{\partial}{\partial t} n\langle A\rangle\right]_{c}
$$

By replacing $A(k)$ by $1, v_{g}(k)$ and $E_{n}(k)$ we obtain the 3 moment equations of the BTE. Table 1 summarizes the results.

\section{REFERENCES}

[1] C. Cercigan ani, The Boltzmann equation and its applications, Springer-Verlag, 1988.

[2] K. Hess, Advanced Theory of Semiconductor Devices, 352p., Wiley-IEEE Press, 1999.

[3] N. Bogoliubov, Problemi dynami, Sov. Phys. JETP, vol. 10, p.256, 1946.

[4] J. R. Barker, "Quantum transport theory of high-field conduction in semiconductors," J. of Physics C: Solid-State Physics, vol. 6, pp.2663-2684, 1973.

[5] C. Buet., S. Dellacherie, R. Sentis, "Numerical solution of an ionic Fokker-Planck equation with electronic temperature", Society for Industrial and Applied Mathematics (SIAM), vol. 39, no.4, pp. 1219-1253, 2001.

[6] A. Arnold, Quantum Fokker-Planck models: Kinetic versus density matrix formulations,[online]. Available: http://mail. math.ups-tlse.fr/ nanolab/Contents/Invited1.pdf, 2004.

[7] F. Capasso, Physics of Quantum Electron Devices, Heidelberg: Sprin ger-Verlag, 1990.

[8] P. Holland, The quantum theory of motion, Cambridge University Press, New York, 1993.

[9] E. Wigner. On the quantum correction for thermodynamic equilibrium. Phys. Rev. vol. 40, pp. 749-759., 1932.

[10] W. Frensley, "Wigner function model of a resonant tunneling semiconductor devices," Phys. Rev. B, vol. 36, no. 3, pp.1570-1580, 1987.

[11] L. Shifren and D. Ferry, "Particle Monte Carlo simulation of Wigner function tunneling," Physics Letters A, vol. 285, pp.217-221, 2001.

[12] T. Shawki, G. Salmer and O. El-Sayed, "2-D simulation of degenerate hot electron transport in MODFET's including DX center trapping," IEEE Trans. CAD, Vol 9, no. 1, pp.11-17, 1990.

[13] M. Cramer and A. Crickenberger, "The Prandtl-Meyer function for dense gases," AIAA Journal, vol. 30, no. 4, pp. 561-564,1992.

[14] A. Abramo et al., "A comparison of numerical solution of the Boltzmann transport equation for high-energy electron transport in Si," IEEE Trans. Electron. Dev., vol.41, no.9, pp. 1646-1654, 1994.

[15] H.D. Rees, "Computer simulation of semiconductor devices," J. Phys. C:Solid-State Phys., vol. 6, pp. 266-273, 1973.

[16] J-P. Aubert, J-C. Vassiere and J-P. Nougier, "Matrix determination of the stationary solution of the Boltzmann Equation for Hot Carriers in Semiconductors," J. Appl. Phys. vol. 56, no. 4, pp.1128-1132, 1984.

[17] W. Fawcett, A. D. Boardman, and S. Swain, "Monte Carlo determination of electron transport properties in Gallium Arsenide," J. Physics and Chemistry of Solids, vol. 31, pp. 1963-1990, 1970.

[18] C. Moglestue, "Monte Carlo particle simulation of hot electron plasma formed in p-n junction," Electron. Lett., vol. 22, p. 397-379, 1972.

[19] C. Jacoboni and P.Lugli, The Monte Carlo method for semiconductor device simulation, Springer-Verlag, Vienna, 1989.

[20] K. Hess Ed., Monte Carlo device simulation: Full band and beyond, Kluwer Academic Press, Boston, 1991.

[21] M. Fischetti, S. Laux, P. Solomon, and A. Kumar, "Thirty years of Monte Carlo simulations of electronic transport in semiconductors: Their relevance to science and mainstream VLSI technology", IBM Research Division, T. J. Watson Research Center, 10598 IWCE 10, Oct. 2004.

[22] M. Saraniti and S. M. Goodnick, "Hybrid fullband cellular automaton/Monte Carlo approach for fast simulation of charge transport in semiconductors," IEEE Trans. Electron Devices, vol. 47, no. 10, 2000

[23] T.-W. Tang and H. Gan, "Two formulations of semiconductor transport equations based on spherical harmonic expansion of the Boltzmann transport equation," IEEE Trans. Electron Devices, vol. 47, no. 9, 2000.

[24] C. Jungemann, B. Meinerzhagen: "A Legendre Polynomial Solver for the Langevin Boltzmann Equation "J. Computational Electronics, vol. 3, pp. 157-160, 2004.

[25] C. Jungemann, Matthias Bollh?fer, B. Meinerzhagen" Convergence of the Legendre

[26] Polynomial Expansion of the Boltzmann Equation for Nanoscale Devices " ESSDERC, pp. 341-344, Grenoble (France), 2005.

[27] C. Auer and F. Schürrer, "Efficient time integration of the Boltzmann-Poisson system applied to semiconductor device 
simulation," J. Computational Electronics, pp. 5-14, vol. 5, no. $1,2006$.

[28] S.F. Liotta and H. Struchtrup, "Moment equations for electrons in semiconductors: Comparison of spherical harmonics and full moments," Solid-State Electronics vol. 44, pp. 95-103, 2000.

[29] R. Stratton, "Diffusion of hot and cold electrons in semiconductor barriers," Physical Review, vol. 126, pp. 2002-2014, 1962.

[30] K. Bløtekjaer, "Transport equations for electrons in two-valley semiconductors," IEEE Trans. Electron devices, vol. ED-17, no. 1, pp. 38-47, 1970.

[31] M.S. Shur and L.F. Eastman, "Near ballistic transport in GaAs devices at 77K," Solid-State Electronics, vol. 24, p. $11,1981$.

[32] J-P. Nougier, J. Vassiere, D. Gasquet, J.Zimmermann and C. Constant, "Determination of the transient regime in semiconductor devices using relaxation time ap proximation," J. Applied Phy sics, vol. 52, pp.825-832, 1981.

[33] R. Cook and J. Frey, "An efficient technique for 2-D simulation of velocity overshoot effects in $\mathrm{Si}$ and $\mathrm{GaAs}$ devices," COMPEL, vol. 1, pp. 65-87, 1982.

[34] G. Baccarani G and M. Wordemann, "An investigation of steady-state velocity overshoot in Silicon", Solid-State Electronics, vol. 28, pp. 407-416, 1985.

[35] C.C. McAndrew, E.L. Heasell and K. Singhal, "A comprehensive transport model for semiconductor device simulation," Semicond. Sci Technology, vol. 2, pp.643-648, 1987.

[36] B. Meinerzhagen and W. Engl, "The influence of the thermal equilibrium approximation on the accuracy of classical 2-D numerical modeling of silicon submicrometer MOS transistors," IEEE Trans. Electron Devices, vol. ED-35, no.5, pp.689-97, 1988.

[37] N. Goldsman and J. Frey, "Efficient use of the energy transp ort method in device simulation," IEEE Trans. Electron Devices, vol. 35, no. 9, 1988.

[38] M. Cheng and Kunhardt, "A theory of non-equilibrium carrier transport in multivalley semiconductors," J. Applied Phy sics, vol. 67 , no. 4, pp. 1907-1914, 1990.

[39] M R. Thoma et al., "Hydrodynamic equations for semiconductors with nonparaboloc band structures," IEEE Trans. on Electron Devices, vol. 38, no. 6, pp.1343-1352, 1991.

[40] D. Chen, E.C. Kan, U. Ravaioli, W.-C Shu and R.W. Dutton, "An improved energy transport model including nonparaboilicty and non-Maxwellian distribution Effects," IEEE Trans. Electron Dev. Lett., vol. 13, no. 1, pp.26-28, 1992.

[41] T-W. Tang and J. Nam, "An improved hydrodynamic transport model for silicon," IEEE Trans. Electron Devices, vol. 40, no. 8, 1993.

[42] C.-S. Yao et al., "Formulation of a tail electron hydrodynamic model based on Monte Carlo results," Electron Dev. Lett., vol. 16, no. 1, pp. 26-29, 1995.
[43] M. Ieong and T-W. Tang, "Influence of hydrodynamic models on the prediction of submicrometer device characteristics," IEEE Trans. on Electron Devices, vol. ED-44, no. 12, 1997.

[44] A. M. Anile, V. Romano and G. Russo, "Extended hy drody namic model of carrier transport in semiconductors," SIAM J. Applied Math, vol. 61, no. 1, pp. 74-101, 2000.

[45] T. Grasser, H. Kossina, M. Gritisch and S. Selberherr, "Using six moments of Boltzmann's transport equation for device simulation," J. Appl. Phys., vol. 90, no. 5, pp.2389-2396, 2001.

[46] M. Gritsch, H. Kosina, T. Grasser, and S. Selberherr, "Revision of the standard hydrodynamic transport model for SOI simulation," IEEE Trans. Electron Devices, vol. ED-49, no. 10, pp.1814-1820, 2002.

[47] T. Grasser. T-W. Tang, H. Kosina and S. Selberherr, "A review of hydrodynamic and energy-transport models for semiconductor device simulation," Proceed in gs of IEEE, vol. 91, no. 2, pp.251-274, 2003.

[48] C.Jungemann, T. Grasser, B.Neinhilus and B.Meinerzhagen, "Failure of moment-based transport models in nanoscale devices near equilibrium," IEEE Trans. Electron Dev., vol. 52 , no. 11, pp. 2404-2408, 2005.

[49] G.K. Batchelor, An Introduction to Fluid Dynamics, Cambridge Univ. Press, New York, 1967.

[50] I. Muller and T. Ruggeri, Extended Thermodynamics, Springer-Verlag, Berlin, 1993.

[51] S. M. Sze, Physics of Semiconductor Devices, Wiley, New York, 1986.

[52] J. Fourier, Théorie Analy tique de la Chaleur, 1822, Reprint ISBN 2-87647-046-2, 670p, Gabay, 1988.

[53] H. Fröhlich, "On the theory of dielectric breakdown in solids," Proc. Roy al Soc., London, vol. I88-A, pp.521-532, 1947.

[54] J. Higman and K. Hess, "Comment on the use of the electron temperature concept for nonlinear transport problems in semiconductor p-n junctions," Solid-State Electronics, vol. 29, no. 9, pp 915-918, 1986.

[55] W. Engl and H.K. Dorks, Models of phy sical parameters, in: An introduction to numerical analysis of semiconductor devices and integrated circuits, Boole Press, Dublin, 1981.

[56] S. Selberherr, On Modeling MOS-Devices, in: Simulation of semiconductor devices, Chapter 8, Elsevier Science Publishers, North-Holland, 1986.

[57] D. Caughey and R. Thomas, "Carrier mobility in silicon empirically related to doping and field," IEEE Proceedings, vol. 54, pp. 2192-2193, 1967.

[58] J.M. Dorkel and Ph. Leturcq, "Carrier mobilities in silicon semi-empirically related to temperature, doping and injection level, Solid-State Electronics, vol. 24, pp. 821-825, 1981.

[59] D. B. Klassen, "A unified mobility model for device simulation-Part I: Model equations and concentration dependence," Solid-State Electron., vol. 35, no. 7, pp.953-959, 1992.

[60] C. Canali,, G. Maijni, R. Minder and G. Ottaviani, "Electron and hole drift velocity measurements in silicon and their 
empirical relation to electric field and temperature," IEEE Trans. Electron Devices, vol. ED-22, pp. 1045-1047, 1975.

[61] M. H. El-Saba, "Accurate modeling of hot-carrier drift mobility in semiconductor devices," Proceedings of the Int. Conf. of Microelectronics ICM VI, Cairo, 1995.

[62] W. Hänsch and M. Miura-Mattausch, "The hot-electron problem in small semiconductor devices," J. Appl. Phy s. vol. 60, pp. 650-656, 1986.

[63] M. H. El-Saba, Modélisation hydrodynamique des phénomènes de transport des porteurs chauds et de l'ionisation par impact dans les dispositives á semiconducteur, PhD Thesis, INSA Lyon, France, Order no. 93 ISAL 0072, 1993.

[64] K.K. Thornber, "Relation of drift velocity to low-field mobility and high-field saturation velocity," J. Appl. Phys., vol. 51, pp. 2127-2136, 1980.

[65] A. G. Sabins and J.T. Clemens, "Characterization of the electron mobility in the inverted (100) Si surface," IEDM Tech. Digest, Dec. 1979, pp. 18-21.

[66] K. Yamaguchi, "A mobility model for carriers in the MOS inversion layer," IEEE Trans. Electron Devices, vol. 30, pp. 658-663, 1983.

[67] S.A. Schwarz et al., "Semi-empirical equations for electron velocity in silicon: Part II- MOS inversion layer," IEEE Trans. Electron Devices, vol. ED-30, no. 12, pp.1634-1639, 1983.

[68] M. Liang, J. Choi, P.K. Ko and C. Hu, "Inversion-layer capacitance and mobility of very thin gate oxide MOSFETs," IEEE Trans. Electron Devices, vol. 33, p. 409, 1986

[69] J. E. Chung, P.K. Ko and C. Hu, "A model for hot-electron induced MOSFET linear-current degradation based on mobility reduction due to interface-state generation," IEEE Trans. Electron Devices, vol. 38, no. 6, pp. 1362-1370, 1991.

[70] S. Takagi, A. Toriumi, M. Iwase and H. Tango, "On the universality of inversion layer mobility in Si. MOSFETs Part I: Effects of substrate impurity concentration," IEEE Trans. Electron Devices, vol. 41, no. 12, pp. 2357-2362,1994.

[71] M. N. Darwish, J. L. Lentz, M. Pinto, P. Zeitzoff, T. J. Krutsick and H. Ha Vuong, "An Improved electron and hole mobility model for general purp ose device simulation," IEEE Trans. Electron Devices, vol. 44, no. 9, pp. 1529-15338, 1997.

[72] C. Lombardi, S. Manzini, A. Saporito and M. Vanzi, "A phy sically-based mobility model for numerical simulation of nonplolar devices," IEEE Trans. Computer-Aided Design, vol. 7, no. 11, pp. 1164-1171, 1988.

[73] K. G. McKay, “Avalanche breakdown in Si,” Phys. Rev. vol. 94, pp.877-884, 1954.

[74] P. A. Wolff, "Theory of multiplication in silicon and germanium,” Phys. Rev., vol. 95, no. 6, pp.1415-1420, 1954.

[75] G.A. Baraff, "Distribution function and ionization rates for hot electrons in semiconductors, Phys. Rev. , vol. 128, no. 6, pp. 2507-2517, 1962.

[76] L. V. Keldish, "Concerning the theory of impact ionization in semiconductors," Sov. Phys. JETP, vol. 21, pp. 1135-1144, 1965.
[77] A. G. Chynoweth, "Ionization rates for electrons and holes in Si,” Phys. Rev. vol. 109, no. 5, pp.1537-1540, 1958.

[78] C. A. Lee, R.A. Logan, R.L. Latdorf, J. Klimack and W. Wiegmann, "Ionization rates of holes and electrons in Si p-n junctions," Phys. Rev. A, vol. 134,pp.761-773, 1964.

[79] R. Van Overstaten and H. De Man, "Measurement of the ionization rates in diffused $\mathrm{Si}$ p-n junctions," Solid-State Electronics, vol. 13, pp.583-608, 1970.

[80] N. Kotani and S. Kawazu, "A Numerical analysis of avalanche breakdown in short-channel MOSFETs," Solid State Electronics, vol. 24, pp. 681-687, 1981.

[81] W. Maes, K. de Meyer, and R. Van Overstraeten, "Impact ionization in silicon: A review and update," Solid-State Electron., vol. 33, pp.705-718, 1990.

[82] I. Takayanagi, K. Matsumoto, and J. Nakamura, "Measurement of electron impact ionization coefficient in bulk silicon under a low-electric field," J. Appl. Phys., vol.72, pp.1989-1992, 1992.

[83] W. Grant, "Electron and hole ionization rates in epitaxial silicon at high electric fields," vol. 16, pp. 1189-1203, 1973.

[84] W. Shockley, "Problems related to p-n junctions in silicon," Solid-state Electronics, vol. 2, pp.35-67, 1961.

[85] S. Tam et al., Hot-electron current in very short channel MOSFETs," IEEE Trans. Electron Dev. Lett., vol. 4,pp.249 251, 1983.

[86] Y-Z. Chen and T-W. Tang, "Numerical simulation of avalan che hot-carrier injection in short-channel MOSFETs," IEEE Trans. Electron Devices, vol. 35, no. 12, 1988.

[87] N. Goldsman, L. Henrickson and J. Frey, "Reconciliation of a hot-electron distribution function with lucky electron exponential model in silicon," IEEE Electron Dev. Let., vol. 11 , no. $10,1990$.

[88] E. Schöll and W. Quade, "Effect of impact ionization on hot-carrier energy and momentum relaxation in Semiconductors”, J. Physics C. Solid-State Phy s., vol. 20,pp. L861-L867, 1987.

[89] W. Quade, E. Schöll, and M. Rudan, "Impact ionization within the hydrodynamic approach to semiconductor transp ort," Solid-State Electronics, vol. 36, no. 10, pp.1493 1505,1993

[90] K. Souissi, F. Odeh, H. Tang, A. Gnudi and P-F. Lu, "Investigation of the impact ionization in the hydrodynamic model," IEEE Trans. Electron Dev. vol. 40, no. 8, pp. 1501 1507,1993

[91] E. Crabbe, J. Stork, G. Baccarani, M. Fischetti and S. Laux, "The impact of non-equilibrium transport on Breakdown in Transit Time in Bipolar Transistors," IEDM Tech. Dig., p.463, 1990.

[92] D. Cassi and B. Ricco, "An analytical model of the energy distribution of hot electrons," IEEE Trans. Electron Devices, vol. 37 , no. 6 , pp. 1514-1521, 1990

[93] K. Matsuzawa, I. Kamohara and T. Wada, "Device simulation including en ergy transport with improved Physical Models," NASECODE VII Trans., J.J. Miller Ed., Colorado, pp.173 $174,1991$. 
[94] C.-S. Yao et al., "Formulation of a tail electron hy drody namic model based on Monte Carlo results," Elec. Dev. Lett., vol. 16, no. 1, pp. 26-29, 1995.

[95] H.J. Peifer, B. Meinerzhagen, R. Thoma and W.L. Engl, "Evaluation of impact ionization modeling in the framework of hydrodynamic equations," IEDM Tech. Digest, 1991.

[96] T. Grasser, H. Kosina and S. Selberherr, "Influence of the distribution function shape and band Structure, on impact ionization modelling," J. Appl. Phys. vol. 90, no. 12, pp. 6165-6171, 2001.

[97] K. Sonoda, S. T. Dunham, M. Yamaji, K. Taniguchi and C. Hamaguchi, "Impact ionization model using average energy and average square energy distribution," Japan. J. Appl. Phys., vol. 35, no. 2B, pp. 818-825, 1996.

[98] T. Grasser, H. Kosina, C. Heitzinger and S. Selberherr, “An impact ionization model including an explicit cold carrier population," Modelling and Simulation of Microsystems, ISBN 0-9708275-7-1, 2002.

[99] H. C. Morris, M. M De Pass and Henok Abebe, "Analy tic formulae for the impact ionization rate for use in compact models of ultra-short semiconductor devices." Proceedings Nanotechnology Conference, vol. 2, pp 140-143, Boston, Massachusetts, USA, 2004.

[100] P. G. Scrobhaci and T.-W. Tang, "Modeling of the hot electron subpopulation and its application to impact ionization in submicron silicon devices- PART I: Transport equations," IEEE Trans. Electron Devices, vol. 41, no.7, p.1197, 1994.

[101] C.S. Yao, J.G. Ahn, Y.-J. Park, H.-S. Min and R.W. Dutton, Formulation of a tail electron hydrodynamic model based on Monte Carlo results," IEEE Electron Devices Lett., vol. 16, no. 1 , p. 26, 1995.

[102] T.-W. Tang and J. Nam, "A simplified impact ionization model on the average energy of hot-electron subpopulation," IEEE Electron Devices Lett., vol. 19, no. 6, 1998.

[103] M. H. El-Saba, “Accurate hy drody namic modeling of impact ionization rate in semiconductor devices," Int. Conf. on Radio Science, URSI, Cairo, 1998.

[104] K. K. Thornber, "Application of scaling to problems in high-field electronic transport," J. Appl. Phys., vol. 52, no. 1, pp. 279-290, 1981.

[105] J. Bude and K. Hess, "Threshold of impact ionization in semiconductors," J. Appl. Phys. vol. 72, no. 8, 1992.

[106] J. Tang, "Theoretical studies of high field transport in GaAs, $\mathrm{Si}$ and heterostructures," $\mathrm{PhD}$ Dissertation, Univ. of Illinois, Urbana, 1983.

[107] C. Crowell and S. M. Sze, "Temperature dependence of avalanche multiplication in semiconductors," Appl. Phys. Lett., vol. 9, no. 6, p. 242, 1966.

[108] R. A. Ballinger, K.G. Major and J.R. Mallinson, "Impact ionization thresholds in semiconductors," J. Phys. C: Solid-state Phys., vol. 6, pp. 2573-2585, 1973.

[109] Ken Kai-fu Chang, Kajen R. S., Shen Chen, Ping Bai, Ganesh Samudra and Erping $\mathrm{Li}$, "Investigation on the Impact Ionization Breakdown Onset of Double-Gate MOSFET structure with Optimized Hy drody namic Model via Full-band
Monte Carlo Method," IEEE Trans. Vol. 46, No. 2, pp. 221-231, 2008.

[110] K. Hess, "Comment on effect of collisional broadening on Monte Carlo simulation of high-field transportin in semiconductor devices," IEEE Electron Devices Lett., Vol EDL-2, pp.297-298, 1989.

[111] F. R. McFeely, E. Cartier and E. Eklund, "New zero-field probes for the transport dynamics of very hot electrons," Proceedings of NASECODE VIII, J.J. Miller (Ed.), Vienna, Austria, pp.42-43, 1992.

[112] W. Quade and Eckehard Scholl, F. Rossi, C. Jacoboni, "Quantum theory of impact ionization in coherent high-field semiconductor transport," The American Physical Society, April 1994

[113] G. Wolodkin and J. Frey, "Overshoot effects in the relaxation time ap proximation," Proceed ingings NASCODE 8, Vienna, 1992, pp. 107-108.

[114] C. Jacoboni and L. Reggiani, "Bulk hot-electron properties of cubic semiconductors," Advanced in Physics, vol. 28, pp. 493-553, 1983.

[115] K. Seeger, Semiconductor Physics, Springer Verlag, 1973.

[116] M. Costato and L. Reggiani, "Electron energy relaxation time in Si and Ge," J. Phys. Chem. Solide, vol. 34, pp.547-564, 1973.

[117] B. Gonzalez et al., "An analytical Energy relaxation time model for device simulation," Solid-State Electron., vol. 43, 1999.

[118] D. Munteanu, G. Le Carval and G. Guegan, "Impact of non-stationary transport effects on realistic 50nm MOS technology," Modelling and Simulation of Microsyatems, 2001.

[119] K. Suzuki, K. Saito, K. Muraki and Y. Hirayama, "Photoluminescence from a modulation-doped A10.33 $\mathrm{Ga} 0.67 \mathrm{As} / \mathrm{GaAs}$ heterointerface under cyclotron resonance" Phys. Rev., vol. B58, p.15385, 1998.

[120] D. M. Rife, J. Optical Soc. America, vol. B 19, p.1092, 2002.

[121] A. J. Sabbah and D. M. Riffe, "Femtosecond pump-probe reflectivity study of silicon carrier dynamics," Phys. Rev. B, vol. 66, pp. 165217-165228, 2002.

[122] A. Matulionis, J. Liberis, L. Eastman, W. Schaff, J. Shealy, X. Chen and Y.-J. Sun, "Electron transp ort and microwave Noise in MBE-and MOCVD-Grown AlGaN/AIN/GaN." Acta Physica Polonica A, vol. 107, pp. 361-364, 2005.

[123] I. Bork, C. Jungemann, B. Meinerzhagen, W. L. Engl, "Influence of heat flux on the accuracy of hydrodynamic models for ultrashort Si MOSFETs," in NUPAD Tech. Dig., Honolulu, 1994.

[124] M. Ieong and T-W. Tang, "Influence of hydrodynamic models on the prediction of submicrometer device characteristics," IEEE Trans. Electron Devices, vol. ED-44, no. 12,1997

[125] M. H. El-Saba, "Problems related to the hydrodynamic model", Proceedings of the 5th Int. Conference on Microelectronics ICM VI, Cairo, 1995.

[126] K. Hasnat, C-F. Yeap, S. Jallepalli, S. A. Hareland, W.K. Shih, V.M. Agostinelli, A.F. tasch and C. M. Maziar, 
"Thermoionic emission model of electron gate current in submicron NMOSFETs," IEEE Trans. Electron Devices, vol, 44, no. 1, pp. 129-138, 1997.

[127] A. Gehring, T. Grasser, and S. Selberherr, "Non-parabolicity and non-Maxwellian effects on gate oxide tunneling, The NSTI Nanotech. Conf. Proceedings, Boston, 2003.

[128] M. Rasras, I. De Wolf, G. Groeseneken, Ben Kaczer, R. Degraeve and H.E. Maes, "Photo-carrier generation as the origin of Fowler-Nordheim-induced substrate hole current in thin oxides," IEEE Trans. Electron Devices, vol. 48, no. 2,pp. 231-238, 2001.

[129] T. H. Ning, C. M. Osburn, and H. N. Yu. Emission probability of hot electrons from silicon into silicon dioxide. J. Appl. Phys., vol. 48, pp. 286-293, 1977.

[130] C. Hu, S. Tam, F. Hsu, P. Ko, T. Chan, and K. Terill, "Hot-electron-induced Mosfet degradation-Model, monitor and improvement," IEEE Trans. Electron Devices, vol. 32, no.2, p. 375-384, 1985.

[131] J. D. Bude, "Gate current by impact ionization feedback in sub-micron MOSFET technologies, " in Proc. Symp. VLSI Tech., 1995, pp. 101-102.

[132] D. R. Nair, S. Shukuri and S. Mahapatra, "Cycling endurance of NOR flash EEPROM cells under CHISEL programming operation-Impact of technology parameters and scaling," IEEE Trans. Electron devices, vol. 51, no. 10, pp. 1672-1678, 2004.

[133] J. Egley, B. Polsky, B. Min, E. Lyumkis, O. Penzin, and M. Foisy, "SOI related simulation challenges with moment-based BTE solvers," Simulation of Semiconductor Processes and Devices, Seattle, Washington, USA, pp. 241-244, Sept. 2000.

[134] M. Gritsch, H. Kosina, T. Grasser, S. Selberherr, T. Linton, S. Singh, S. Yu, and M.D. Giles, "The failure of the hydrodynamic transport model for simulation of partially depleted SOI MOSFETs and its revision," in SIO Technology and Devices X, (Washington DC, USA), pp. 181-186, 2001.

[135] E. Pop, K. Banerjee, Per Sverdrup, R. Dutton and K. Goodson, "Localized heating effects and scaling of Sub-0.18 micron CMOS devices," Int. Electron Devices Meeting (IEDM), 2002.

[136] M. Cardona, Fundamentals of Semiconductors, Springer Verlag, Berlin, 1996.

[137] M-Y Chuang and M. E. Law, "A new algorithm for faster full-thermodynamic device simulations," IEEE Trans. Electron Devices, vol. 44, no. 9, 1997

[138] C. Kittle, Introduction to Solid-State Physics, New York, Wiley \& Sons, 1967.

[139] A. Chryssafis and W.A. Love, "Computer-aided analy sis of one-dimensional thermal transients in n-p-n Power transistors," Solid-state Electron. vol. 22, pp. 249-256, 1979.

[140] J. C. Glassbenner and G.A. Slack, "Thermal conductivity of silicon and germanium from $3 \mathrm{~K}$ to the melting point," Phys. Rev. A, vol. 134, pp. 1058-1069, 1964.

[141] P. Su, K-I. Goto, T. Sugii, and C. Hu, "A thermal activation view of low voltage impact ionization in MOSFETs," IEEE Electron Devices letters, vol. 23, no. 9, pp.550- 552, 2002.
[142] M. Asheghi, Touzelbaev, M.N., K.E. Goodson, Y.K.Leung and S.S.Wong, "Temperature-dependent thermalconductivit y of single-crystal silicon layers in SOI substrates," ASME Journal of Heat Transfer, vol. 120, pp. 31-36, 1998.

[143] P. G. Sverdrup, O. Tornblad, K. Banerjee, D. Yergeau, Z. Yu, R. W. Dutton and K. E. Goodson, "Advanced electro-thermal modeling and simulation Techniques for deep Submicron devices," Techcon, 2000.

[144] D. A. Romanov, B. A. Glavin, and V. V. Mitin, "Stimulated decay of non-selectively pumped optical phonons in GaAs," Phys. Rev. B vol. 60, no. 7, 1999.

[145] C. Gardner, "The quantum hydrodynamic model for semiconductor devices," SIAM Journal on Applied Mathematics, vol. 54, pp. 409-427, 1994.

[146] D. Ferry, H. Grubin, "Modelling of quantum transport in semiconductor devices," Solid State Phys. 49 , pp.283-448, 1995.

[147] I. Burghardt, S. Lorenz, S. Cederbaum, K. B. Moller, G. Parlant, Hydrodynamic methods for ultrafast quantum dynamics, quantum transport, and dissipation,[online]. A vailable: http://mail.math.ups-tlse.fr/ nanolab/Contents/ Invited2.pdf , 2004.

[148] E. Madelung, "Quantentheorie in hydrodynamischer form," Z. Phy sik, vol. 40,pp.322-326, 1927.

[149] Ansgar J.ungel and D. Matthes1, "A derivation of the isothermal quantum hydrodynamic equations using entropy minimization," Appl. Numer. Math, 2005.

[150] D. Ferry and J.-R. Zhou, "Form of the quantum potential for use in hydrodynamic equations for semiconductor device modeling," Phys. Rev. B, vol. 48,pp. 7944-7950, 1993.

[151] C. Gardner and C. Ringhofer, "The smooth quantumpotential for the hydrody namic Model," Phys. Rev. vol. E53, pp.157 $167,1996$.

[152] C.S. Rafferty et al., "Multi-dimensional quantum effect simulation using a density-gradient model and script-level programming techniques," SISPAD'98, Leuven, Belgium, Sept., 1998, p.137.

[153] D. Connelly, Z. Yu and D. Yergeau, "Macroscopic simulation of quantum mechanical effects in 2-D MOS devices via the density gradient method," IEEE Trans. vol. 49, no. 4, pp. 619-626, 2002.

[154] H.U. Baranger and J.W. Wilkins, "Ballistic structure in the electron distribution function of small semiconducting structures: General features and specific trends," Phys. Rev. B, vol. 36, pp.1487-1502, 1987.

[155] M.S. Lundstrom, "Elementary scattering theory of the MOSFET,” IEEE Electron Dev. Lett., vol. 18, pp.361-363, 1997.

[156] M. Lundstrom and Zhibin Ren, "Essential physics of carrier transport in nanoscale MOSFETs," IEEE Trans. Electron Devices, vol. 49, no. 1, 2002.

[157] K. Banoo, J.-H. Rhew, M. Lundstrom, C.-W Shu and J. W. Jerome, "Simulating quasi-ballistic transport in $\mathrm{Si}$ nanotransistors," 7th Int. Workshop Computational Electronics, IWCE, July, 2000. 
[158] W. C. Leonard, F. Register and S.K. Banerjee, Simulation of quantum effects along the channel of ultrathin Si-based MOSFETs," IEEE Trans. vol. 49, no. 4, pp. 652-657, 2002

[159] A. Svizhenko and M.P. Arantvan, "Role of scattering in nanotransistors," IEEE Trans. Vol. 50, no. 6, pp. 1459-1466, 2003.

[160] M. H. El-Saba, "Investigation of the hot carriers rebelling effect in semiconductor devices, Using an Analy tical Solution of the Hydrody namic Model," IEEE Trans. Electron Devices, vol. 52, no. 7, pp. 1561-1568, 2006.

[161] Shiyu Chen, Kunyuan Xu, and Gang Wang, "Monte Carlo Investigation of Size-Dependent Impact Ionization Properties in InP Under Submicron Scale, Journal of Lightwave Technology, Vol. 27, Issue 10, pp. 1347-1354, 2009. 